\title{
Grenvillian magmatism in the northern Virginia Blue Ridge: Petrologic implications of episodic granitic magma production and the significance of postorogenic A-type charnockite
}

\author{
Richard P. Tollo ${ }^{\mathrm{a}, *}$, John N. Aleinikoff ${ }^{\mathrm{b}}$, Elizabeth A. Borduas ${ }^{\mathrm{a}}$, \\ Alan P. Dickin ${ }^{c}$, Robert H. McNutt ${ }^{\text {c }}$, C. Mark Fanning ${ }^{d}$ \\ a Department of Earth and Environmental Sciences, George Washington University, Washington, DC 20052, USA \\ ${ }^{\mathrm{b}}$ U.S. Geological Survey, Denver, CO 80225, USA \\ ${ }^{\mathrm{c}}$ School of Geography and Geology, McMaster University, Hamilton, Ontario L8S 4M1, Canada \\ ${ }^{\mathrm{d}}$ Research School of Earth Sciences, Australian National University, Mills Road, \\ Canberra, ACT 0200, Australia
}

Received 29 May 2006; received in revised form 23 August 2006; accepted 31 August 2006

\begin{abstract}
Grenvillian ( 1.2 to $1.0 \mathrm{Ga}$ ) plutonic rocks in northern Virginia preserve evidence of episodic, mostly granitic magmatism that spanned more than 150 million years (m.y.) of crustal reworking. Crystallization ages determined by sensitive high resolution ion microprobe (SHRIMP) U-Pb isotopic analyses of zircon and monazite, combined with results from previous studies, define three periods of magmatic activity at 1183-1144 Ma (Magmatic Interval I), 1120-1111 Ma (Magmatic Interval II), and 1078-1028 Ma (Magmatic Interval III). Magmatic activity produced dominantly tholeiitic plutons composed of (1) low-silica charnockite, (2) leucogranite, (3) non-leucocratic granitoid (with or without orthopyroxene (opx)), and (4) intermediate biotite-rich granitoid. Field, petrologic, geochemical, and geochronologic data indicate that charnockite and non-charnockitic granitoids were closely associated in both space and time, indicating that presence of opx is related to magmatic conditions, not metamorphic grade. Geochemical and $\mathrm{Nd}$ isotopic data, combined with results from experimental studies, indicate that leucogranites (Magmatic Intervals I and III) and non-leucocratic granitoids (Magmatic Intervals I and II) were derived from parental magmas produced by either a high degree of partial melting of isotopically evolved tonalitic sources or less advanced partial melting of dominantly tonalitic sources that also included a more mafic component. Post-orogenic, circa $1050 \mathrm{Ma}$ low-silica charnockite is characterized by A-type compositional affinity including high $\mathrm{FeOt} /(\mathrm{FeOt}+\mathrm{MgO}), \mathrm{Ga} / \mathrm{Al}, \mathrm{Zr}, \mathrm{Nb}, \mathrm{Y}$, and $\mathrm{Zn}$, and was derived from parental magmas produced by partial melting of potassic mafic sources in the lower crust. Linear geochemical trends defined by leucogranites, low-silica charnockite, and biotite-rich monzogranite emplaced during Magmatic Interval III reflect differences in source-related characteristics; these features do not represent an igneous fractionation sequence. A compositional gap between circa 1160 Ma magnesian low-silica charnockite and penecontemporaneous higher silica lithologies likewise precludes a fractionation relationship among plutons intruded during Magmatic Interval I. Correspondence in timing of magmatic activity between the Blue Ridge and neighboring Mesoproterozoic terranes underscores the widespread nature of Grenvillian processes in the region.
\end{abstract}

Published by Elsevier B.V.

Keywords: A-type; Charnockite; SHRIMP; Grenville; Blue Ridge; Nd isotopes

\footnotetext{
* Corresponding author. Tel.: +1 202994 6960; fax: +1 2029940450.

E-mail address: rtollo@gwu.edu (R.P. Tollo).
} 


\section{Introduction}

Orogenic belts typically contain abundant evidence of the extended sequence of tectonic processes that result in their formation. Deciphering this geological record is an important means of elucidating tectonic mechanisms and, especially for Precambrian orogens, reconstructing the geologic history of early Earth. However, in areas where cyclic plate tectonic processes locally superpose the effects of subsequesent orogenic and other events such as rifting on previously activated crust, geologic evidence of the earliest tectonic activity can be obscured. Nevertheless, investigations involving detailed field studies and application of modern analytical techniques (e.g. Frost et al., 2000; Slagstad et al., 2004) have demonstrated the efficacy of multidisciplinary studies of the oldest rocks in polydeformed orogenic belts to produce useful insight into the nature of ancient tectonic processes.

This study presents results of detailed field mapping and petrologic, geochemical, and geochronologic investigations of Mesoproterozoic crust in the northern Blue Ridge. It expands the findings of Tollo et al. (2004a) by presenting results from a much larger field area encompassing previously unreported rock types. This report includes 135 major- and trace-element analyses of samples from all lithologic units and new geochronologic data from 12 additional samples. The research is designed to elucidate the nature and sequence of Mesoproterozoic tectonomagmatic events involved in the geologic evolution of Blue Ridge basement and to evaluate the significance of these events within the wider context of the Grenville orogen. A major emphasis is the application of detailed geochronologic data to temporally calibrate and improve understanding of petrologic and tectonic processes. In this paper, the terms "Grenville orogeny" and "Grenvillian orogenesis" refer to largely compressional tectonism that occurred at $1.2-1.0 \mathrm{Ga}$ and which, in the Adirondacks and Grenville province of southeastern Canada, involved multiple pulses including Ottawan activity which is most closely correlative temporally to events recorded in the northern Blue Ridge.

The largely granitic Mesoproterozic rocks of the northern Blue Ridge record an interval of magmatism and tectonic activity spanning more than 150 million years (m.y.) (Aleinikoff et al., 2000; Tollo et al., 2004a) Field relations and results from geochronologic investigations indicate that magmatic activity both predated and postdated a major episode of deformation. As a result, these rocks offer a rare perspective on Grenvillian geodynamic activity and shed light on the role of granitic magmatism in the evolution of orogens.

\section{Regional geology}

The Blue Ridge province is an inlier of Grenvilleage basement located within the Paleozoic Appalachian orogen of central and northern Virginia (Fig. 1). This area preserves evidence of multiple tectonic cycles spanning more than 1 billion year and includes collisional orogens of both Mesoproterozoic (Grenvillian) and Paleozoic (Appalachian) age. Mesoproterozoic rocks of the Blue Ridge and other Appalachians inliers are especially noteworthy because they occur in an area where rocks of the Grenville orogen are otherwise buried beneath Phanerozoic sediments in eastern North America south of the Adirondacks (Fig. 1).

The Blue Ridge in Virginia is a fault-bounded allochthonous thrust sheet that was transported northwestward to its present location during the Carboniferous-Permian Alleghenian orogeny (Rankin et al., 1989). This sheet is the largest of about 12 external (allochthonous) and internal (autochthonous) massifs that contain Grenvillian basement within the Appalachian orogen from Alabama to Newfoundland (Fig. 1). Mesoproterozoic basement rocks in Virginia occur within the core of the northeast-trending, northwest-vergent Blue Ridge anticlinorium (Fig. 2). They are locally intruded by numerous Neoproterozoic extension-related A-type plutons such as the 735-700 Ma Robertson River batholith (Tollo and Aleinikoff, 1996), and are structurally overlain by the circa $570 \mathrm{Ma}$ extension-related metavolcanic and metasedimentary Catoctin Formation and underlying metasedimentary units (Rankin et al., 1989; Aleinikoff et al., 1995).

The igneous and metamorphic basement rocks of the Virginia Blue Ridge were formed at $1.2-1.0 \mathrm{Ga}$ (Aleinikoff et al., 2000; Tollo et al., 2004a) during Grenvillian orogenesis associated with tectonic assembly of Rodinia (Dalziel, 1997; Karlstrom et al., 2001). The Grenville orogeny in Virginia involved polyphase deformation, high-grade metamorphism, and abundant, largely granitic plutonism, resulting in construction of a terrane composed primarily of granitoids and charnockites that locally enclose isolated screens and inliers of granitic gneiss (Bartholomew and Lewis, 1984; Tollo et al., 2004a). Orthopyroxene (opx) + garnet assemblages occurring in Blue Ridge gneisses (Sinha and Bartholomew, 1984; Tollo et al., 2004b) indicate that ambient conditions of Grenvillian orogenesis locally reached granulite facies (Spear, 1993). Most rocks also display mineralogic evidence of limited retrograde recrystallization at greenschist-facies conditions resulting from multiple Paleozoic orogenies (Kunk and 


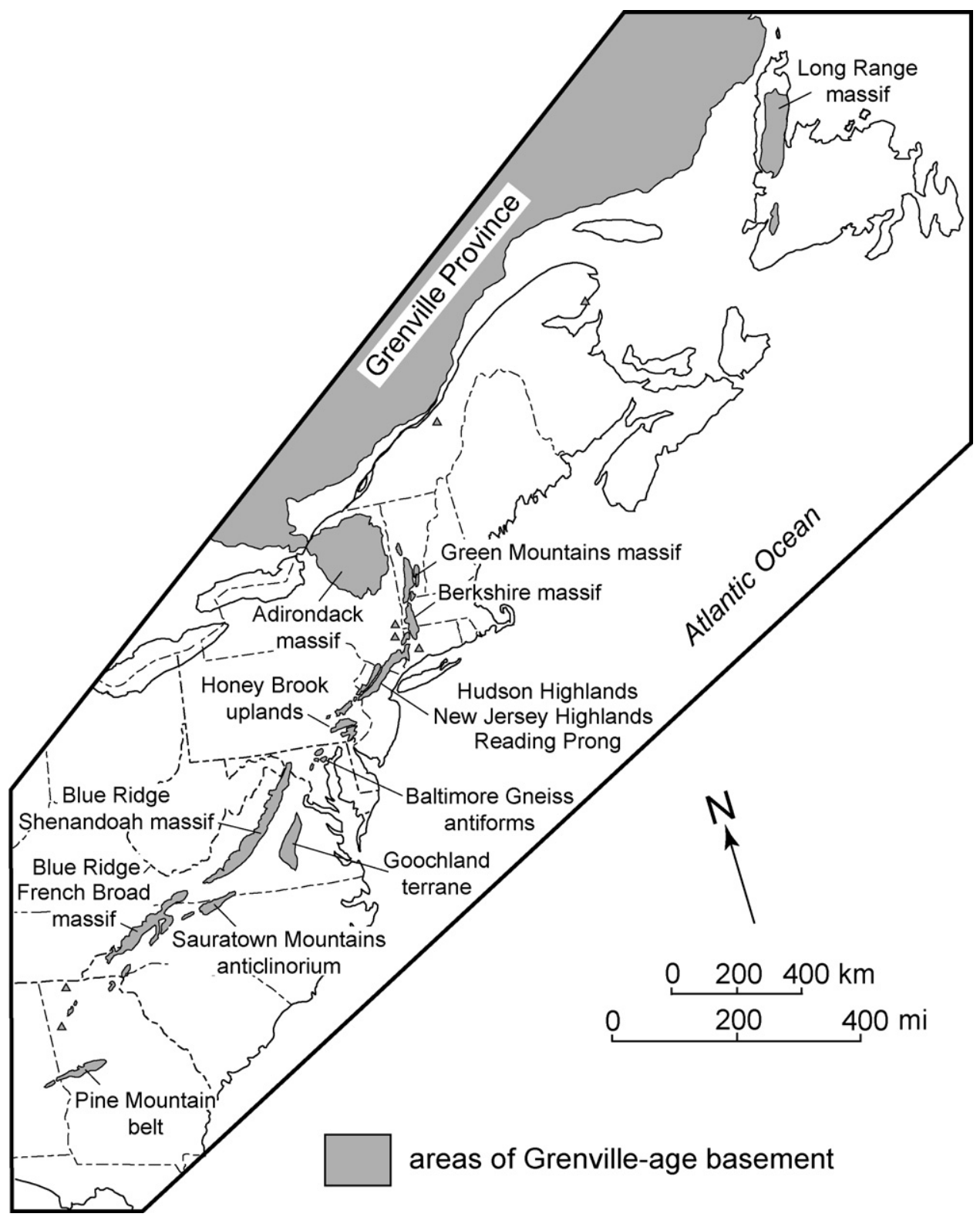

Fig. 1. Map showing locations of major occurrences of Grenville-age basement in eastern North America. Map modified after Rankin et al. (1990).

Burton, 1999). Paleozoic orogenesis also resulted in widespread ductile deformation manifested by numerous high-strain zones (Fig. 3) that affected basement rocks throughout the Blue Ridge (Bailey and Simpson, 1993) but involved displacement on the order of only a few kilometers (Bailey et al., 1994).

\section{Previous studies}

Basement rocks of the northern Virginia Blue Ridge are dominated by granitic gneisses and compositionally variable granitioids that include abundant opx-bearing (charnockitic) types (Bartholomew and Lewis, 1984). Early studies indicated that charnockitic rocks ("Pedlar Formation" of Bloomer and Werner, 1955) were concentrated in western portions of the area, whereas amphibole \pm biotite-bearing rocks of presumably lower metamorphic grade dominated eastern regions ("Lovingston Formation" of Jonas, 1928). Bartholomew (1977) and Bartholomew et al. (1981) subdivided the Blue Ridge into two massifs: (1) the upper granulite facies Pedlar massif located west of the northeast-trending Rock- 


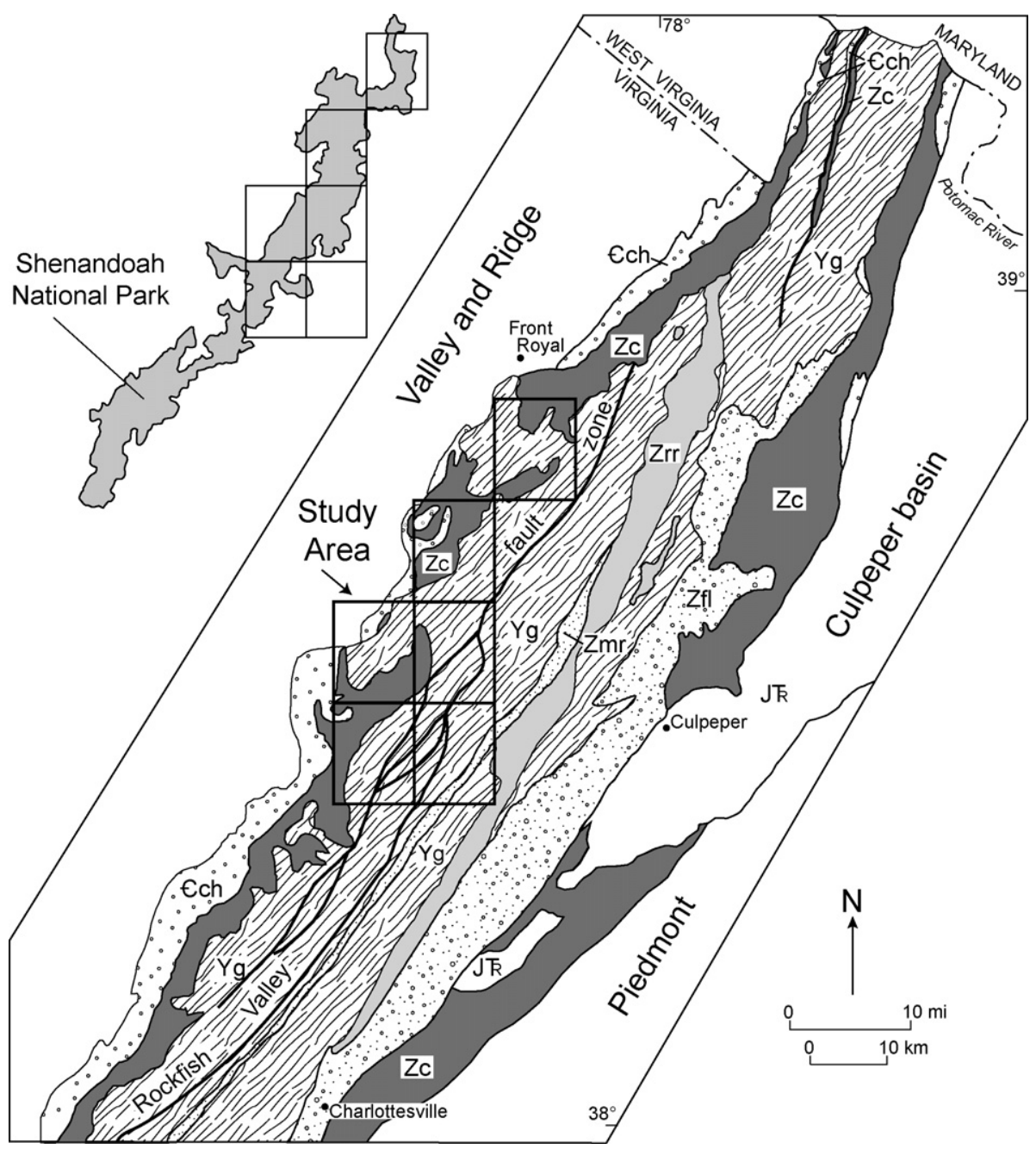

Fig. 2. Generalized geologic map of the Blue Ridge anticlinorium in northern Virginia showing location of study area included in Fig. 3. Modified after Virginia Division of Mineral Resources (1993). Faults shown only in Mesoproterozoic rocks. Insert map shows location of Shenandoah National Park.

fish Valley fault zone (Fig. 2) and (2) the lower granulite to upper amphibolite facies Lovingston massif located east of the fault. In this model, the Rockfish Valley fault zone represents a significant structural boundary in central Virginia along which the two massifs were juxtaposed by Paleozoic thrusting (Sinha and Bartholomew, 1984). Evans' (1991) alternative model attributes lithologic differences to hydration and retrograde metamorphism of Lovingston rocks resulting from fluid migration associated with Neoproterozoic extension and Paleozoic orogenesis. Subsequent studies suggest that (1) rocks of the two massifs are grossly similar in geochemical composition and thus unlikely to represent greatly disparate crustal blocks (Hughes et al., 2004), (2) movement along the fault zone is likely insufficient to juxtapose crustal blocks (Bailey and Simpson, 1993), and (3) most lithologic variation in northern Virginia reflects primary mineralogy of igneous plutons (this study).

Field-based petrologic and structural studies indicate that northern Blue Ridge basement is composed of a complex array of granitic rocks that preserve evidence of over 150 m.y. of magmatic and metamorphic processes (Aleinikoff et al., 2000; Tollo et al., 2004a,c). The oldest rocks are represented by strongly foliated granitoids and granitic gneisses that are locally intruded by, and enclosed within, younger magmatic rocks. The older rocks range from quartz diorite to syenogranite (Table 1) and are characterized by (1) deformed compositional layering that commonly is defined by discontinuous leucosomes, (2) pervasive alignment of primary minerals, and (3) open to isoclinal folds (e.g. Burton et al., 1995; Bailey 


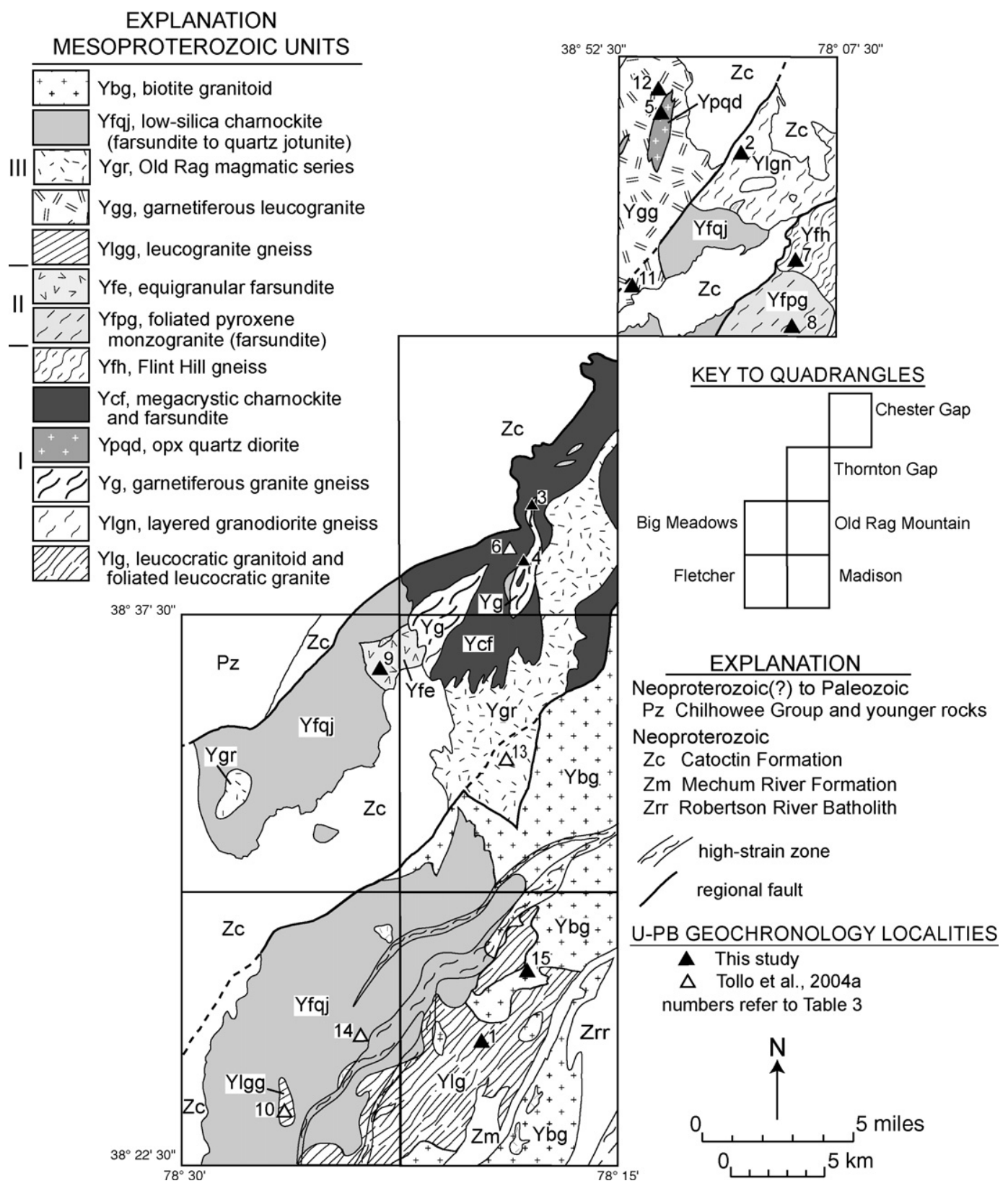

Fig. 3. Generalized geologic map of the study area. Geology of the Madison quadrangle is from Bailey et al. (2003).

et al., 2003; Burton and Southworth, 2004; Southworth, 1995). Younger rocks range from quartz monzodiorite to syenogranite (Table 1); these rocks are variably foliated but do not display fabrics indicative of deformation at high-grade conditions.

Results from previous $\mathrm{U}-\mathrm{Pb}$ geochronologic studies of zircon and monazite indicate that igneous crystallization ages range from circa 1160 to $1050 \mathrm{Ma}$ (Aleinikoff et al., 2000; Tollo et al., 2004a), defining three episodes of magmatic activity occurring at circa $1160-1140 \mathrm{Ma}$
(Magmatic Interval I), circa $1112 \mathrm{Ma}$ (Magmatic Interval II), and 1078-1050 Ma (Magmatic Interval III). Field relations and ages of crosscutting igneous units indicate that a major deformational event occurred between 1078 and $1050 \mathrm{Ma}$ (Tollo et al., 2004a), approximately contemporaneous with the Ottawan orogeny documented elsewhere (McLelland et al., 2001; Rivers, 1997). New mapping and analytical data of this study refine the previous three-fold temporal division of Grenvillian igneous activity in the northern Blue Ridge. 
Table 1

Lithologic characteristics of northern Blue Ridge basement rocks

\begin{tabular}{|c|c|c|c|c|c|}
\hline Magmatic Interval & Map symbol & Lithologic nomenclature $^{\mathrm{a}}$ and group ${ }^{\mathrm{b}}$ & Fabric and rock type & Primary $\mathrm{Fe}-\mathrm{Mg}$ minerals ${ }^{\mathrm{c}}$ & Field relations \\
\hline \multirow[t]{5}{*}{ III (1078-1028 Ma) } & Ybg & $\begin{array}{l}\text { Biotite-rich monzogranite to quartz } \\
\text { monzodiorite (BM) }\end{array}$ & $\begin{array}{l}\text { Variably foliated, bt-rich; local } \\
\text { compositional layering; } \\
\text { granitoid }\end{array}$ & bt & $\begin{array}{l}\text { Intrudes and includes xenoliths } \\
\text { of Ylg }\end{array}$ \\
\hline & Yfqj & $\begin{array}{l}\text { Monzogranite (farsundite) to quartz } \\
\text { monzodiorite (quartz jotunite) (LSC) }\end{array}$ & Non-foliated; charnockite & $\mathrm{opx} \pm \mathrm{amph} \pm \mathrm{cpx}$ & Intruded by Ygr; intrudes Ylgg \\
\hline & Ygr & $\begin{array}{l}\text { Old Rag magmatic series } \\
\text { (garnetiferous leucogranite) (LG) }\end{array}$ & $\begin{array}{l}\text { Non-foliated to weakly } \\
\text { foliated; leucogranite }\end{array}$ & $\mathrm{bt} \pm \mathrm{grt} \pm \mathrm{opx}$ & Intrudes Yfqj and Ycf \\
\hline & Ygg & Garnetiferous syenogranite (LG) & Weakly foliated; leucogranite & $g r t+b t \pm o p x(p s)^{d}$ & Intrudes Ypqd \\
\hline & Ylgg & Megacrystic leucogranite gneiss (LG) & Strongly foliated; leucogranite & bt & Intruded by Yfqj \\
\hline \multirow[t]{2}{*}{ II (1120-1111 Ma) } & Yfe & $\begin{array}{l}\text { Equigranular pyroxene monzogranite } \\
\text { (farsundite) (NLG) }\end{array}$ & $\begin{array}{l}\text { Weakly to moderately foliated; } \\
\text { charnockite }\end{array}$ & $\mathrm{opx} \pm \mathrm{bt} \pm \mathrm{grt}$ & - \\
\hline & Yfpg & $\begin{array}{l}\text { Foliated pyroxene monzogranite } \\
\text { (farsundite) (NLG) }\end{array}$ & Strongly foliated; charnockite & $\mathrm{opx} \pm \mathrm{amph} \pm \mathrm{bt} \pm \mathrm{grt}$ & Contact truncates foliation in Yfh \\
\hline \multirow[t]{6}{*}{ I (1183-1144 Ma) } & Yfh & Flint Hill Gneiss (syenogranite) (NLG) & Strongly foliated; granitoid & bt & - \\
\hline & Ycf & $\begin{array}{l}\text { Megacrystic syenogranite and } \\
\text { monzogranite (charnockite and } \\
\text { farsundite) (NLG) }\end{array}$ & Strongly foliated; charnockite & $\mathrm{opx} \pm \mathrm{amph} \pm \mathrm{cpx}$ & $\begin{array}{l}\text { Intruded by Ygr; encloses screen } \\
\text { of } \mathrm{Yg}\end{array}$ \\
\hline & Ypqd & $\begin{array}{l}\text { Pyroxene quartz diorite (orthopyroxene } \\
\text { quartz diorite) (LSC) }\end{array}$ & Strongly foliated; charnockite & $o p x+b t$ & Intrudes Ylgn; intruded by Ygg \\
\hline & $\mathrm{Yg}$ & $\begin{array}{l}\text { Garnetiferous granite (charnockite to } \\
\text { farsundite) gneiss (NLG) }\end{array}$ & $\begin{array}{l}\text { Strongly foliated, } \\
\text { compositional layering; } \\
\text { charnockite }\end{array}$ & $o p x+g r t+b t \pm c p x$ & Forms screen within Ycf \\
\hline & Ylgn & $\begin{array}{l}\text { Layered granodiorite (opdalite) gneiss } \\
\text { (NLG) }\end{array}$ & $\begin{array}{l}\text { Strongly foliated, } \\
\text { compositional layering; } \\
\text { charnockite }\end{array}$ & opx + amph & Intruded by Ypqd \\
\hline & Ylg & $\begin{array}{l}\text { Leucocratic granitoid (Ylg2) (LG) and } \\
\text { foliated megacrystic leucocratic granite } \\
(\mathrm{Ylg} 1)(\mathrm{LG})\end{array}$ & $\begin{array}{l}\text { Strongly to weakly foliated; } \\
\text { leucogranite }\end{array}$ & bt & $\begin{array}{l}\text { Intruded by Ybg; Ylg2 intrudes } \\
\text { Ylg1; leucogranite pegmatite } \\
\text { intrudes both Ylg1 and Ylg2 }\end{array}$ \\
\hline
\end{tabular}

${ }^{a}$ Nomenclature for charnockitic rocks are italized in parentheses, following recommendations of Le Maitre et al. (1989).

${ }^{\mathrm{b}}$ Lithologic groups: LG: leucogranite, NLG: non-leucocratic granitoid (includes charnockites), LSC: low-silica charnockite; BM: biotite monzogranite.

c Abbreviations after Kretz (1983): bt, biotite; opx, orthorhombic pyroxene; amph, amphibole; cpx, monoclinic pyroxene; grt, garnet.

${ }^{\mathrm{d}}$ ps: pseudomorphed by retrograde metamorphic mineral assemblage. 


\section{Lithologic characteristics and field relations}

The study area includes six contiguous 7.5-min quadrangles located in the core of the Blue Ridge anticlinorium in northern Virginia (Figs. 2 and 3). Previous studies demonstrated the existence of multiple lithologies, including granitic gneisses, charnockite, and leucocratic granitoids of Grenvillian affinity (Gathright, 1976; Virginia Division of Mineral Resources, 1993; Lukert, 1982; Sinha and Bartholomew, 1984).

\subsection{Normative compositions and nomenclature}

All rocks are interpreted to be igneous in origin based on (1) primary mineral assemblages (Table 1); (2) chemical compositions compared to experimental data and compositions of granitic rocks of igneous origin from other orogens (e.g. Rämö, 1991; Kilpatrick and Ellis, 1992; Landenberger and Collins, 1996; Smith et al., 1999); (3) igneous-type textures observed in the least deformed rocks; (4) field relations including crosscutting contacts, incorporation of xenoliths, and local development of cogenetic pegmatite; (5) systematic variation trends in chemical compositions indicative of igneous processes, and (6) concentric oscillatory compositional zoning patterns of zircon from most rocks. Normative compositions, calculated assuming $\mathrm{Fe}^{2+} / \mathrm{Fe}_{\text {total }}=0.9$, are used in place of modal data for determining lithologic nomenclature because of the very coarse grain size and strongly anisotropic fabrics of some rocks. Standard International Union of Geological Sciences (IUGS) nomenclature (Streckeisen and Le Maitre, 1979;
Le Maitre et al., 1989) is used for all rocks (Table 1; Fig. 4). However, specialized nomenclature, recommended for opx-bearing rocks of inferred igneous origin (i.e. charnockites, sensu lato), is employed for some units and is included in italics. Such terms include charnockite (opx-bearing syenogranite), farsundite (opx-bearing monzogranite), opdalite (opx-bearing granodiorite), and quartz jotunite (opx-bearing quartz monzodiorite). The term "charnockitic" is used to refer to all opx-bearing granitoid rocks of inferred igneous origin.

Normative compositions define a broad range extending from alkali feldspar granite to quartz diorite (Fig. 4); however, the extent of compositional variation is strongly dependent on age. Rocks emplaced during Magmatic Intervals I and II are dominantly monzogranite and syenogranite; a notable exception are rocks from a small body of pyroxene quartz diorite (Ypqd) that occurs as an inlier within younger leucogranitoid (Fig. 3). In contrast, rocks emplaced during Magmatic Interval III define a broad and continuous span of compositions, ranging from alkali feldspar granite to quartz monzodiorite (Fig. 4).

\subsection{Field relations}

Geologic mapping indicates that the present erosional surface is a crustal cross section characterized by repeated emplacement of Mesoproterozoic magmas. Supracrustal rocks are rare throughout the Virginia Blue Ridge (Bartholomew and Lewis, 1984; Rankin et al., 1989). Field relations and geochronologic data define an intrusive sequence for rocks of the study area (Table 1;

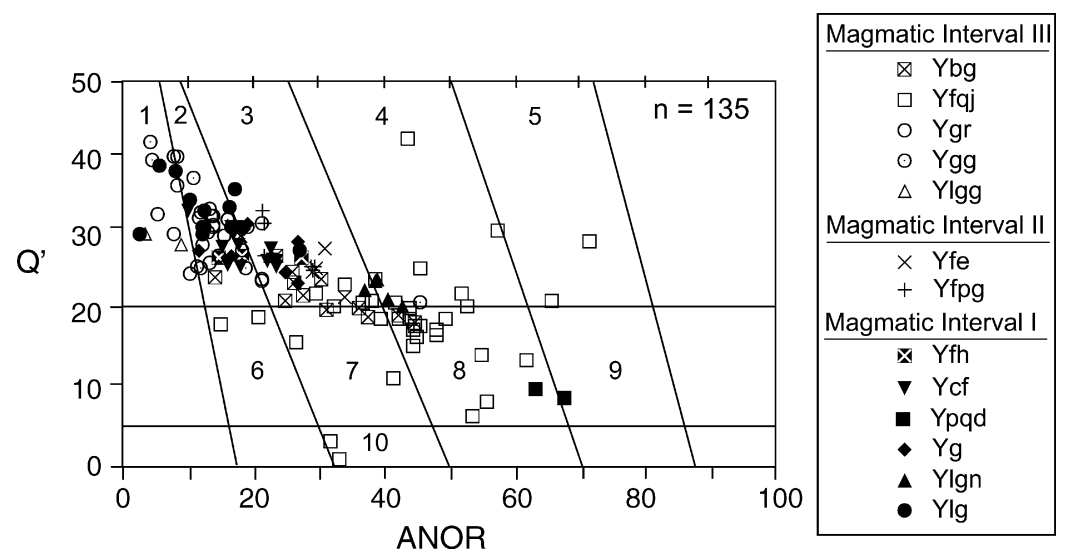

Fig. 4. Plot of normative [anorthite/(orthoclase + anorthite) $\times 100$ vs. normative [quartz/(quartz + orthoclase + albite + anorthite) $\times 100($ ANOR vs. $\mathrm{Q}^{\prime}$, after Streckeisen and Le Maitre, 1979). Normative compositions calculated with $\mathrm{Fe}^{2+} / \mathrm{Fe}_{\text {total }}=0.9$. See Table 1 for explanation of map symbols for lithologic units. Rocks emplaced during Magmatic Interval I are indicated by filled symbols, Magmatic Interval II by line symbols, and Magmatic Interval III by unfilled symbols. Numbered field labels include (1) alkali feldspar granite, (2) syenogranite, (3) monzogranite, (4) granodiorite, (5) tonalite, (6) quartz syenite, (7) quartz monzonite, (8) quartz monzodiorite, (9) quartz diorite, and (10) monzodiorite. 
Fig. 3). Rocks of Magmatic Intervals I and II predate regional high-grade deformation and typically display strong foliation and/or gneissosity. Younger rocks lack ductile fabrics, exhibiting instead variably developed foliation due to waning stages of Grenvillian activity. All rocks were affected by low-grade Paleozoic metamorphism and structural overprinting. The rocks are divisible on the basis of mineralogic and geochemical composition into four lithologic types including (1) leucogranites (feldspar + quartz $>90$ modal \%; units Ylg, Ylgg, Ygg, and Ygr), (2) non-leucocratic granitoids that include both orthopyroxene-bearing types (charnockites, sensu lato) and non-orthopyroxene-bearing types (units Ylgn, Yg, Ycf, Yfh, Yfe, and Yfpg), (3) lowsilica $\left(<65 \% \mathrm{SiO}_{2}\right)$ charnockites (units Ypqd and Yfqj), and (4) biotite-rich monzogranite (Ybg). Charnockitic rocks were emplaced during nearly the full range of local Mesoproterozoic igneous activity. Such plutons typically include co-magmatic dikes and, in some instances (e.g. Yfqj), pegmatite. Leucogranite plutons emplaced during Magmatic Intervals I and III are typically internally complex, characterized by multiple crosscutting lithologies including leucogranitic pegmatites (Bailey et al., 2003; Tollo et al., 2004c). Biotite-rich monzogranite (Ybg) (Magmatic Interval III) is intermediate between the silica-rich and silica-poor rocks (Fig. 4).

Some gneissic units (e.g. Yg and Ylgg) occur as large xenoliths within younger plutons (Fig. 3). Tollo et al. (2004a) reported an igneous crystallization age of $1078 \pm 9$ Ma for strongly deformed leucogranite gneiss (Ylgg) that occurs as xenoliths within relatively undeformed low-silica charnockite (Yfqj) (1050 8 Ma). These relations define the timing of a local pulse of Grenvillian deformation that corresponds to the age of Ottawan orogenesis in the Adirondacks (McLelland et al., 2001) and southeastern Canada (Rivers, 1997). Locally, peak deformation affected rocks as young as the oldest pluton of Magmatic Interval III (Ylgg), developing strong ductile fabrics; waning stages affected rocks as young as $1028 \mathrm{Ma}$, typically imparting weak foliation.

\section{Geochronology}

\subsection{Analytical methods}

Relatively unweathered samples of about $10 \mathrm{~kg}$ were collected from outcrops of nine lithologic units (Fig. 3). Zircon and monazite were extracted by routine mineral separation processes, including crushing, pulverizing, Wilfley table concentration, and purification using magnetic separation and methylene iodide. Individual grains were handpicked under a binocular microscope, mounted in epoxy, ground to nearly half thickness, and polished using 6 and $1 \mu \mathrm{m}$ diamond suspension. All grains were digitally imaged in both transmitted and reflected light to discern areas free from inclusions, cracks, and other imperfections. Using the scanning electron microscope, zircons were imaged by cathodoluminescence (CL), whereas monazites were imaged by back-scattered electrons (BSE).

Zircons in these rocks preserve multiple age zones indicative of complex histories involving igneous origin, inheritance from source materials, and episodes of metamorphic overprinting (Tables 2 and 3). To decipher these events, we determined $\mathrm{U}-\mathrm{Pb}$ ages of small, homogeneous areas of grains by in situ microanalysis using the sensitive high resolution ion microprobe (SHRIMP II) at the Research School of Earth Sciences, Australian National University, following the methods of Williams (1998). The area excavated by the primary oxygen ion beam is about $25-30 \mu \mathrm{m}$ in diameter for zircon and about $15-20 \mu \mathrm{m}$ for monazite. ${ }^{206} \mathrm{~Pb} /{ }^{238} \mathrm{U}$ ages were corrected for instrument fractionation using zircon standard R33 ( $419 \pm 1 \mathrm{Ma}$; Black et al., 2004) or monazite standard 44069 (424 $\pm 1 \mathrm{Ma}$; Aleinikoff et al., 2006). Uranium concentrations are considered accurate to $\pm 20 \%$. Monazite was analyzed using energy filtering (Rubatto et al., 2001) to avoid isobaric interference at mass 204 (Ireland et al., 1999). Raw data were reduced using Squid 1 (Ludwig, 2001) and plotted using Isoplot 3 (Ludwig, 2003). All isotopic data (shown as 2-sigma error ellipses) are plotted on concordia diagrams to visually determine coherent age groups. Ages are calculated using the weighted average of ${ }^{207} \mathrm{~Pb} /{ }^{206} \mathrm{~Pb}$ ages. A Concordia Age (Ludwig, 1998) was calculated in cases where several analyses form a concordant age group.

Age data are reported for 12 new samples from nine lithologic units: (1) biotite-rich monzogranite to quartz monzodiorite (Ybg), (2) garnetiferous syenogranite (Ygg), (3) equigranular pyroxene monzogranite (Yfe), (4) foliated pyroxene monzogranite (Yfpg), (5) Flint Hill Gneiss (Yfh), (6) orthopyroxene quartz diorite (Ypqd), (7) garnetiferous granite gneiss (Yg), (8) layered granodiorite gneiss (Ylgn), and (9) leucocratic granitoid and foliated megacrystic leucocratic granite (Ylg2 and Ylg1, respectively). U-Pb geochronologic data obtained by conventional isotopic techniques were previously reported for the Flint Hill Gneiss (Lukert et al., 1977). However, because of internal compositional complexity of zircons from this unit, we decided to analyze another sample collected from a different locality in order to make use of the high spatial resolution of the SHRIMP technique. 
Table 2

SHRIMP U-Th- $\mathrm{Pb}$ data for zircon and monazite from rocks of the northern Blue Ridge, Virginia

\begin{tabular}{|c|c|c|c|c|c|c|c|c|c|c|c|c|c|c|c|}
\hline $\begin{array}{l}\begin{array}{l}\text { Sample } \\
\text { (location) }\end{array} \\
\end{array}$ & $\begin{array}{c}\text { Measured } \\
{ }^{204} \mathrm{~Pb} / 206 \mathrm{~Pb}\end{array}$ & $\begin{array}{c}\text { Measured } \\
{ }^{207} \mathrm{~Pb} /{ }^{206} \mathrm{~Pb}\end{array}$ & $\begin{array}{l}\text { \% Common } \\
{ }^{206} \mathrm{~Pb}\end{array}$ & $\begin{array}{c}\mathrm{U} \\
(\mathrm{ppm})\end{array}$ & $\mathrm{Th} / \mathrm{U}$ & $\begin{array}{c}{ }^{206} \mathrm{~Pb}^{\mathrm{b} / 238} \mathrm{U} \\
(\mathrm{Ma})\end{array}$ & $\begin{array}{l}\mathrm{Err}^{\mathrm{c}} \\
(\mathrm{Ma})\end{array}$ & $\begin{array}{c}{ }^{207} \mathrm{~Pb}^{\mathrm{b}} / 206 \mathrm{~Pb} \\
(\mathrm{Ma})\end{array}$ & $\begin{array}{l}\mathrm{Err}^{\mathrm{c}} \\
\text { (Ma) }\end{array}$ & $\begin{array}{c}\text { Disc. } \\
\%\end{array}$ & ${ }^{207} \mathrm{~Pb}^{\mathrm{d} / 235} \mathrm{U}$ & $\begin{array}{l}\text { Err }^{\mathrm{c}} \\
(\%)\end{array}$ & ${ }^{206} \mathrm{~Pb}^{\mathrm{d} / 238} \mathrm{U}$ & $\begin{array}{l}\text { Err }^{\mathrm{c}} \\
(\%)\end{array}$ & $\begin{array}{l}\text { Err } \\
\text { corr }\end{array}$ \\
\hline \multicolumn{16}{|c|}{ SNP-02-186 biotite monzogranite (Ybg), Madison quadrangle, UTM coordinates: Easting ${ }^{\mathrm{e}}: 0735$ 611, Northing ${ }^{\mathrm{e}}: 4259886$} \\
\hline $02-186-1.1 \mathrm{c}$ & 0.000105 & 0.0759 & 0.34 & 30 & 0.68 & 1017.3 & 15.5 & 1054 & 55 & 3 & 1.76 & 3.2 & 0.1712 & 1.6 & 0.501 \\
\hline $02-186-2.1 \mathrm{c}$ & -0.000646 & 0.0736 & -0.12 & 19 & 0.71 & 1058.3 & 20.5 & 1262 & 103 & 16 & 2.05 & 5.6 & 0.1801 & 2 & 0.361 \\
\hline $02-186-2.2 \mathrm{r}$ & 0.00011 & 0.0751 & 0.12 & 75 & 0.24 & 1045.8 & 11.4 & 1030 & 47 & -2 & 1.79 & 2.6 & 0.176 & 1.1 & 0.445 \\
\hline $02-186-3.1 \mathrm{c}$ & 0.000321 & 0.0736 & 0 & 30 & 0.7 & 1030.3 & 16.4 & 899 & 97 & -15 & 1.64 & 5 & 0.1724 & 1.7 & 0.336 \\
\hline $02-186-4.1 \mathrm{c}$ & 0.000186 & 0.075 & 0.07 & 23 & 0.72 & 1053.6 & 18.7 & 996 & 76 & -6 & 1.77 & 4.2 & 0.1771 & 1.8 & 0.443 \\
\hline $02-186-5.1 \mathrm{c}$ & 0.000028 & 0.0753 & 0.18 & 52 & 0.45 & 1036.7 & 13.8 & 1066 & 57 & 3 & 1.8 & 3.1 & 0.1747 & 1.4 & 0.433 \\
\hline $02-186-6.1 \mathrm{c}$ & 0.00083 & 0.0772 & 0.44 & 28 & 0.83 & 1029.3 & 17.1 & 783 & 124 & -31 & 1.54 & 6.2 & 0.1714 & 1.8 & 0.286 \\
\hline $02-186-7.1 \mathrm{c}$ & 0.00012 & 0.0735 & 0.12 & 29 & 0.64 & 1000.6 & 16.3 & 980 & 64 & -2 & 1.66 & 3.6 & 0.1678 & 1.7 & 0.475 \\
\hline $02-186-8.1 \mathrm{c}$ & -0.000097 & 0.0759 & 0.32 & 27 & 0.88 & 1020.5 & 17.1 & 1128 & 44 & 10 & 1.84 & 2.8 & 0.1724 & 1.7 & 0.618 \\
\hline $02-186-9.1 c$ & -0.000385 & 0.0717 & -0.13 & 18 & 0.87 & 1006.8 & 20 & 1125 & 84 & 11 & 1.81 & 4.7 & 0.1699 & 2.1 & 0.443 \\
\hline $02-186-10.1 \mathrm{c}$ & -0.000035 & 0.0766 & 0.42 & 31 & 0.73 & 1017.9 & 16.1 & 1125 & 39 & 10 & 1.83 & 2.5 & 0.1719 & 1.6 & 0.644 \\
\hline $02-186-11.1 \mathrm{c}$ & -0.000314 & 0.0774 & 0.52 & 22 & 0.8 & 1014.2 & 18.9 & 1241 & 64 & 18 & 1.94 & 3.8 & 0.1722 & 1.9 & 0.508 \\
\hline $02-186-12.1 \mathrm{c}$ & 0.000007 & 0.0753 & 0.29 & 69 & 0.29 & 1010.8 & 11.9 & 1074 & 34 & 6 & 1.76 & 2.1 & 0.1702 & 1.2 & 0.58 \\
\hline $02-186-13.1 \mathrm{c}$ & 0.000486 & 0.0759 & 0.25 & 24 & 0.69 & 1036.3 & 18.1 & 896 & 137 & -16 & 1.65 & 6.9 & 0.1734 & 1.9 & 0.272 \\
\hline $02-186-13.2 \mathrm{r}$ & 0.000379 & 0.0743 & 0.2 & 56 & 0.34 & 1004.4 & 12.6 & 895 & 64 & -12 & 1.59 & 3.4 & 0.1678 & 1.3 & 0.39 \\
\hline $02-186-14.1 \mathrm{c}$ & 0.000336 & 0.0735 & 0.04 & 34 & 0.73 & 1018.7 & 15.4 & 889 & 92 & -15 & 1.61 & 4.7 & 0.1703 & 1.6 & 0.336 \\
\hline $02-186-14.2 \mathrm{r}$ & 0.000459 & 0.0756 & 0.38 & 51 & 0.38 & 997.4 & 13.1 & 900 & 69 & -11 & 1.59 & 3.6 & 0.1667 & 1.4 & 0.381 \\
\hline $02-186-15.1 \mathrm{c}$ & 0.000011 & 0.072 & -0.07 & 69 & 0.3 & 1002.3 & 11.8 & 982 & 28 & -2 & 1.67 & 1.8 & 0.1681 & 1.2 & 0.666 \\
\hline $02-186-16.1 \mathrm{c}$ & 0.000123 & 0.0746 & 0.06 & 17 & 0.5 & 1044.4 & 21.7 & 1010 & 73 & -3 & 1.76 & 4.2 & 0.1756 & 2.2 & 0.513 \\
\hline $02-186-16.2 \mathrm{r}$ & -0.000011 & 0.0739 & -0.03 & 67 & 0.32 & 1046.7 & 12.2 & 1043 & 26 & 0 & 1.8 & 1.7 & 0.1763 & 1.2 & 0.687 \\
\hline \multicolumn{16}{|c|}{ SNP-02-197 garnetiferous syenogranite (Ygg), Chester Gap quadrangle, UTM coordinates: Easting: 0740 365, Northing: 4304228} \\
\hline 02-197-1.1r & 0.000009 & 0.0749 & 0.01 & 1272 & 0.01 & 1027.4 & 9.3 & 1062 & 10 & 3 & 1.78 & 1.1 & 0.173 & 0.9 & 0.889 \\
\hline 02-197-2.1r & 0.000009 & 0.0765 & 0.02 & 1073 & 0.01 & 1075 & 12.4 & 1105 & 11 & 3 & 1.91 & 1.3 & 0.1817 & 1.2 & 0.91 \\
\hline $02-197-3.1 \mathrm{r}$ & 0.000002 & 0.0736 & 0 & 1430 & 0.03 & 1015.7 & 6.8 & 1029 & 7 & 1 & 1.73 & 0.8 & 0.1708 & 0.7 & 0.899 \\
\hline $02-197-4.1 \mathrm{c}$ & 0.000018 & 0.0754 & 0.03 & 1031 & 0.28 & 1073.1 & 6.5 & 1071 & 8 & 0 & 1.88 & 0.7 & 0.1811 & 0.6 & 0.846 \\
\hline $02-197-2.2 \mathrm{c}$ & 0.000007 & 0.0776 & 0.01 & 1089 & 0.41 & 1115.8 & 6.7 & 1134 & 7 & 2 & 2.02 & 0.7 & 0.1891 & 0.6 & 0.857 \\
\hline $02-197-3.2 \mathrm{c}$ & 0.000032 & 0.0775 & 0.05 & 1283 & 0.27 & 1151.3 & 6.8 & 1121 & 7 & -3 & 2.07 & 0.7 & 0.1953 & 0.6 & 0.865 \\
\hline $02-197-4.2 \mathrm{c}$ & 0.000051 & 0.0759 & 0.09 & 1787 & 0.27 & 987.6 & 5.8 & 1074 & 7 & 8 & 1.72 & 0.7 & 0.1662 & 0.6 & 0.872 \\
\hline $02-197-5.1 \mathrm{r}$ & 0.000054 & 0.0736 & 0.09 & 878 & 0.03 & 1000.5 & 6.1 & 1009 & 10 & 1 & 1.69 & 0.8 & 0.1679 & 0.6 & 0.778 \\
\hline $02-197-5.2 \mathrm{c}$ & 0.000152 & 0.0794 & 0.26 & 1188 & 0.29 & 1076.7 & 6.4 & 1128 & 10 & 5 & 1.94 & 0.8 & 0.1822 & 0.6 & 0.781 \\
\hline $02-197-6.1 \mathrm{c}$ & 0.000006 & 0.0749 & 0.01 & 1818 & 0.28 & 1055.7 & 6.2 & 1063 & 6 & 1 & 1.84 & 0.7 & 0.178 & 0.6 & 0.899 \\
\hline $02-197-7.1 \mathrm{c}$ & 0.000003 & 0.0777 & 0 & 989 & 0.31 & 1136.9 & 6.8 & 1137 & 7 & 0 & 2.06 & 0.7 & 0.1929 & 0.6 & 0.858 \\
\hline $02-197-7.2 \mathrm{c}$ & 0.000004 & 0.0784 & 0.01 & 1244 & 0.29 & 1154.3 & 6.8 & 1156 & 7 & 0 & 2.12 & 0.7 & 0.1961 & 0.6 & 0.882 \\
\hline $02-197-8.1 \mathrm{c}$ & 0.000006 & 0.0755 & 0.01 & 1561 & 0.16 & 1076.6 & 7 & 1080 & 12 & 0 & 1.89 & 0.9 & 0.1818 & 0.7 & 0.755 \\
\hline $02-197-9.1 \mathrm{c}$ & 0.000015 & 0.0781 & 0.03 & 843 & 0.24 & 1142.8 & 7 & 1144 & 8 & 0 & 2.08 & 0.8 & 0.194 & 0.6 & 0.836 \\
\hline $02-197-10.1 \mathrm{c}$ & 0.00002 & 0.0774 & 0.03 & 1563 & 0.39 & 1103.4 & 6.5 & 1125 & 7 & 2 & 1.99 & 0.7 & 0.1869 & 0.6 & 0.864 \\
\hline $02-197-11.1 \mathrm{c}$ & 0.000056 & 0.075 & 0.09 & 1191 & 0.26 & 1004.6 & 6 & 1048 & 9 & 4 & 1.73 & 0.8 & 0.169 & 0.6 & 0.81 \\
\hline $02-197-12.1 \mathrm{c}$ & 0.000002 & 0.0764 & 0 & 1219 & 0.2 & 1111.2 & 6.7 & 1106 & 10 & 0 & 1.98 & 0.8 & 0.1881 & 0.6 & 0.788 \\
\hline 02-197-13.1c & 0.000011 & 0.076 & 0.02 & 1982 & 0.25 & 1087.7 & 6.3 & 1092 & 7 & 0 & 1.92 & 0.7 & 0.1838 & 0.6 & 0.873 \\
\hline $02-197-14.1 \mathrm{c}$ & 0.000007 & 0.0764 & 0.01 & 1430 & 0.35 & 1079.7 & 6.4 & 1104 & 6 & 2 & 1.92 & 0.7 & 0.1825 & 0.6 & 0.888 \\
\hline $02-197-15.1 \mathrm{c}$ & 0.000061 & 0.077 & 0.1 & 820 & 0.23 & 1038.8 & 6.3 & 1098 & 11 & 5 & 1.84 & 0.8 & 0.1753 & 0.6 & 0.751 \\
\hline $02-197-16.1 \mathrm{r}$ & 0.000012 & 0.0742 & 0.02 & 452 & 0.04 & 1025.9 & 6.7 & 1043 & 12 & 2 & 1.76 & 0.9 & 0.1726 & 0.7 & 0.757 \\
\hline $02-197-17.1 \mathrm{r}$ & 0.000314 & 0.0781 & 0.53 & 408 & 0.05 & 1007.8 & 6.7 & 1033 & 25 & 2 & 1.72 & 1.4 & 0.1694 & 0.7 & 0.492 \\
\hline 02-197-18.1r & 0.000026 & 0.0748 & 0.04 & 496 & 0.04 & 1140 & 7.8 & 1054 & 12 & -8 & 1.98 & 0.9 & 0.1927 & 0.7 & 0.758 \\
\hline 02-197-19.1r & -0.000047 & 0.0745 & -0.08 & 436 & 0.04 & 1044.6 & 6.9 & 1074 & 16 & 3 & 1.83 & 1 & 0.1761 & 0.7 & 0.654 \\
\hline $02-197 \mathrm{~m}-1.1$ & 0.000027 & 0.0733 & 0.05 & 398 & 22.8 & 1116.9 & 16.1 & 1011 & 17 & -10 & 1.89 & 1.7 & 0.1883 & 1.5 & 0.866 \\
\hline $02-197 \mathrm{~m}-1.2$ & -0.000023 & 0.0748 & -0.04 & 634 & 15 & 1054.1 & 13 & 1071 & 12 & 2 & 1.84 & 1.4 & 0.1778 & 1.3 & 0.908 \\
\hline $02-197 \mathrm{~m}-1.3$ & 0.000006 & 0.0741 & 0.01 & 479 & 13.7 & 1040.5 & 15.7 & 1043 & 16 & 0 & 1.79 & 1.8 & 0.1752 & 1.6 & 0.887 \\
\hline $02-197 \mathrm{~m}-2.1$ & -0.00003 & 0.0738 & -0.05 & 463 & 19.5 & 1057.9 & 13.2 & 1048 & 16 & -1 & 1.82 & 1.5 & 0.1783 & 1.3 & 0.847 \\
\hline $02-197 \mathrm{~m}-2.2$ & -0.000028 & 0.0748 & -0.05 & 504 & 15.1 & 1070.4 & 13.7 & 1074 & 14 & 0 & 1.87 & 1.5 & 0.1807 & 1.3 & 0.88 \\
\hline
\end{tabular}




\begin{tabular}{|c|c|c|c|c|c|c|c|c|c|c|c|c|c|c|c|}
\hline $02-197 \mathrm{~m}-3.1$ & 0.000027 & 0.0745 & 0.05 & 547 & 9.6 & 1097.2 & 13 & 1043 & 14 & -5 & 1.89 & 1.4 & 0.1851 & 1.2 & 0.868 \\
\hline $02-197 \mathrm{~m}-3.2$ & 0.000011 & 0.0749 & 0.02 & 827 & 10.2 & 1076.2 & 12 & 1062 & 14 & -1 & 1.87 & 1.3 & 0.1816 & 1.2 & 0.861 \\
\hline $02-197 \mathrm{~m}-4.1$ & 0 & 0.0774 & 0 & 1261 & 5.1 & 1160.6 & 12 & 1132 & 16 & -3 & 2.1 & 1.4 & 0.197 & 1.1 & 0.791 \\
\hline $02-197 \mathrm{~m}-4.2$ & 0.000012 & 0.0741 & 0.02 & 556 & 14.7 & 1040.7 & 12.4 & 1040 & 14 & 0 & 1.79 & 1.4 & 0.1752 & 1.2 & 0.869 \\
\hline $02-197 \mathrm{~m}-4.3$ & 0.000029 & 0.075 & 0.05 & 898 & 11 & 1046.6 & 11.4 & 1057 & 11 & 1 & 1.81 & 1.3 & 0.1764 & 1.1 & 0.892 \\
\hline $02-197 \mathrm{~m}-5.1$ & -0.000009 & 0.0756 & -0.02 & 732 & 13.9 & 1054.4 & 17.9 & 1087 & 12 & 3 & 1.86 & 1.9 & 0.178 & 1.8 & 0.946 \\
\hline $02-197 \mathrm{~m}-5.2$ & 0.000035 & 0.0744 & 0.06 & 430 & 23.8 & 1001.4 & 12.9 & 1038 & 17 & 4 & 1.71 & 1.6 & 0.1683 & 1.3 & 0.847 \\
\hline $02-197 \mathrm{~m}-6.1$ & 0 & 0.0748 & 0 & 795 & 7.8 & 1097 & 12.2 & 1062 & 12 & -3 & 1.91 & 1.3 & 0.1852 & 1.2 & 0.896 \\
\hline $02-197 \mathrm{~m}-6.2$ & 0.000015 & 0.0741 & 0.03 & 417 & 23.1 & 1024.1 & 13.2 & 1039 & 18 & 1 & 1.76 & 1.6 & 0.1723 & 1.3 & 0.831 \\
\hline $02-197 \mathrm{~m}-7.1$ & -0.000007 & 0.0742 & -0.01 & 855 & 9.8 & 1054.1 & 11.7 & 1049 & 11 & 0 & 1.82 & 1.3 & 0.1776 & 1.1 & 0.897 \\
\hline $02-197 \mathrm{~m}-7.2$ & -0.000021 & 0.0757 & -0.04 & 541 & 11.2 & 1048 & 12.9 & 1096 & 15 & 4 & 1.85 & 1.5 & 0.1769 & 1.3 & 0.86 \\
\hline $02-197 \mathrm{~m}-8.1$ & 0 & 0.0757 & 0 & 534 & 17.5 & 1109.7 & 13.6 & 1087 & 14 & -2 & 1.96 & 1.5 & 0.1877 & 1.3 & 0.87 \\
\hline $02-197 \mathrm{~m}-8.2$ & 0.000021 & 0.0732 & 0.04 & 627 & 13.4 & 1025.3 & 14.7 & 1012 & 14 & -1 & 1.73 & 1.6 & 0.1723 & 1.5 & 0.907 \\
\hline $02-197 \mathrm{~m}-9.1$ & 0.000012 & 0.0743 & 0.02 & 409 & 27.3 & 1033.9 & 21.7 & 1044 & 18 & 1 & 1.78 & 2.3 & 0.174 & 2.2 & 0.928 \\
\hline $02-197 \mathrm{~m}-9.2$ & 0.000015 & 0.0748 & 0.03 & 693 & 10.5 & 1055.8 & 12.3 & 1056 & 13 & 0 & 1.83 & 1.4 & 0.178 & 1.2 & 0.882 \\
\hline $02-197 \mathrm{~m}-9.3$ & 0.000017 & 0.075 & 0.03 & 770 & 8.6 & 1138.4 & 12.9 & 1062 & 12 & -7 & 1.98 & 1.3 & 0.1925 & 1.2 & 0.894 \\
\hline $02-197 \mathrm{~m}-10.1$ & 0.000058 & 0.0735 & 0.1 & 461 & 19.2 & 1032.4 & 14.9 & 1005 & 22 & -3 & 1.74 & 1.9 & 0.1735 & 1.5 & 0.804 \\
\hline $02-197 \mathrm{~m}-10.2$ & 0.000042 & 0.0752 & 0.07 & 716 & 13.7 & 1060 & 12.4 & 1057 & 15 & 0 & 1.84 & 1.4 & 0.1787 & 1.2 & 0.851 \\
\hline $02-197 \mathrm{~m}-11.1$ & 0.000009 & 0.075 & 0.02 & 1092 & 6.7 & 1067.5 & 11.4 & 1065 & 10 & 0 & 1.86 & 1.2 & 0.1801 & 1.1 & 0.908 \\
\hline $02-197 \mathrm{~m}-11.2$ & 0 & 0.0731 & 0 & 577 & 15.4 & 1049.3 & 12.6 & 1018 & 14 & -3 & 1.78 & 1.4 & 0.1765 & 1.2 & 0.871 \\
\hline $02-197 \mathrm{~m}-12.1$ & 0.000019 & 0.0754 & 0.03 & 1065 & 16.4 & 1051 & 11.4 & 1072 & 12 & 2 & 1.84 & 1.3 & 0.1772 & 1.1 & 0.888 \\
\hline $02-197 \mathrm{~m}-12.2$ & 0 & 0.0725 & 0 & 430 & 17.5 & 1007.6 & 13 & 999 & 17 & -1 & 1.69 & 1.6 & 0.1691 & 1.3 & 0.854 \\
\hline \multicolumn{16}{|c|}{ SNP-01-170 garnetiferous syenogranite (Ygg), Chester Gap quadrangle, UTM coordinates: Easting: 0739 690, Northing: 4295280} \\
\hline $01-170-1.1 \mathrm{c}$ & 0.004467 & 0.1329 & 7.4 & 516 & 1.34 & 961.6 & 11.5 & 894 & 293 & -8 & 1.52 & 14.2 & 0.1604 & 1 & 0.069 \\
\hline $01-170-2.1 \mathrm{c}$ & 0.000387 & 0.0816 & 0.83 & 124 & 1.06 & 1059.1 & 9.5 & 1098 & 46 & 4 & 1.88 & 2.5 & 0.1789 & 0.9 & 0.381 \\
\hline $01-170-3.1 \mathrm{c}$ & 0.005663 & 0.158 & 9.88 & 327 & 1.18 & 1071.6 & 18.2 & 1139 & 330 & 6 & 1.94 & 16.7 & 0.1814 & 1.3 & 0.079 \\
\hline $01-170-3.2 \mathrm{r}$ & 0.000365 & 0.0775 & 0.81 & 2072 & 0.06 & 949.8 & 11.6 & 993 & 22 & 4 & 1.58 & 1.7 & 0.159 & 1.3 & 0.755 \\
\hline $01-170-4.1 \mathrm{c}$ & 0.001034 & 0.0957 & 1.98 & 147 & 0.41 & 1171.2 & 16.1 & 1224 & 61 & 4 & 2.23 & 3.4 & 0.1997 & 1.4 & 0.413 \\
\hline $01-170-5.1 \mathrm{c}$ & 0.000023 & 0.0798 & -0.06 & 461 & 0.26 & 1206.1 & 10.3 & 1185 & 8 & -2 & 2.25 & 1 & 0.2055 & 0.9 & 0.911 \\
\hline $01-170-6.1 \mathrm{r}$ & 0.000323 & 0.075 & 0.96 & 2089 & 0.06 & 838.3 & 6.1 & 939 & 22 & 11 & 1.35 & 1.3 & 0.1394 & 0.7 & 0.576 \\
\hline $01-170-7.1 \mathrm{r}$ & 0.000226 & 0.0748 & 0.98 & 2671 & 0.06 & 825.7 & 6.6 & 972 & 14 & 15 & 1.36 & 1.1 & 0.1375 & 0.8 & 0.762 \\
\hline 01-170-8.1r & 0.000187 & 0.0733 & 0.63 & 1972 & 0.07 & 871.5 & 9.8 & 946 & 14 & 8 & 1.41 & 1.4 & 0.1452 & 1.2 & 0.857 \\
\hline \multicolumn{16}{|c|}{ SNP-03-204 equigranular pyroxene monzogranite (farsundite) ( $\mathrm{Yfe}$ ), Big Meadows } \\
\hline $03-204-1.1 \mathrm{c}$ & 0.000084 & 0.0767 & 0.3 & 133 & 0.3 & 1045.4 & 9.2 & 1082 & 17 & 3 & 1.84 & 1.3 & 0.1763 & 0.9 & 0.723 \\
\hline $03-204-2.1 \mathrm{c}$ & 0.000014 & 0.0768 & -0.35 & 179 & 0.3 & 1191.5 & 22.8 & 1110 & 48 & -7 & 2.14 & 3.1 & 0.2022 & 2 & 0.631 \\
\hline $03-204-3.1 \mathrm{c}$ & 0.000001 & 0.0824 & -0.18 & 172 & 0.4 & 1290.1 & 11.2 & 1254 & 21 & -3 & 2.51 & 1.4 & 0.2212 & 0.9 & 0.647 \\
\hline $03-204-4.1 \mathrm{c}$ & 0.000007 & 0.0821 & 0.17 & 116 & 0.73 & 1213.3 & 14.1 & 1246 & 41 & 3 & 2.35 & 2.4 & 0.2074 & 1.2 & 0.489 \\
\hline $03-204-5.1 \mathrm{c}$ & 0.00001 & 0.0861 & -0.51 & 163 & 0.48 & 1434.2 & 16.1 & 1336 & 45 & -7 & 2.94 & 2.6 & 0.2479 & 1.1 & 0.439 \\
\hline $03-204-6.1 \mathrm{c}$ & 0.00002 & 0.0799 & 0.32 & 241 & 0.42 & 1125.5 & 10.8 & 1187 & 11 & 5 & 2.1 & 1.1 & 0.1913 & 1 & 0.869 \\
\hline $03-204-7.1 \mathrm{c}$ & 0.000043 & 0.0744 & -0.15 & 169 & 0.34 & 1085.5 & 14.8 & 1035 & 21 & -5 & 1.86 & 1.8 & 0.183 & 1.4 & 0.8 \\
\hline $03-204-8.1 \mathrm{c}$ & 0.000025 & 0.0766 & 0.17 & 162 & 0.36 & 1072.9 & 10.2 & 1100 & 16 & 2 & 1.9 & 1.3 & 0.1813 & 1 & 0.772 \\
\hline $03-204-9.1 \mathrm{c}$ & 0.000008 & 0.0774 & 0.24 & 208 & 0.37 & 1077.7 & 10.7 & 1128 & 14 & 4 & 1.94 & 1.2 & 0.1824 & 1 & 0.832 \\
\hline $03-204-9.2 \mathrm{r}$ & 0.000014 & 0.0748 & 0.27 & 803 & 0.23 & 1003.1 & 7.7 & 1059 & 24 & 5 & 1.74 & 1.4 & 0.1688 & 0.8 & 0.545 \\
\hline $03-204-10.1 \mathrm{c}$ & 0.000025 & 0.0781 & 0.22 & 208 & 0.45 & 1100.3 & 15.1 & 1140 & 13 & 3 & 2 & 1.6 & 0.1865 & 1.4 & 0.911 \\
\hline $03-204-11.1 \mathrm{c}$ & 0.000069 & 0.0786 & 0.38 & 168 & 0.34 & 1080.7 & 10 & 1138 & 27 & 5 & 1.96 & 1.7 & 0.183 & 1 & 0.569 \\
\hline 03-204-12.1r & -0.000003 & 0.0754 & -0.07 & 423 & 0.23 & 1095.5 & 16.6 & 1081 & 15 & -1 & 1.93 & 1.7 & 0.1851 & 1.6 & 0.904 \\
\hline $03-204-12.2 \mathrm{c}$ & -0.000014 & 0.0743 & 0.12 & 161 & 0.23 & 1021.2 & 15.6 & 1054 & 14 & 3 & 1.77 & 1.7 & 0.1719 & 1.6 & 0.911 \\
\hline $03-204-13.1 \mathrm{c}$ & 0.000017 & 0.0775 & 0.25 & 144 & 0.32 & 1079.7 & 10.2 & 1129 & 20 & 4 & 1.95 & 1.4 & 0.1827 & 1 & 0.698 \\
\hline $03-204-14.1 \mathrm{c}$ & 0.000018 & 0.0762 & 0.42 & 108 & 0.39 & 1006.7 & 9.7 & 1094 & 32 & 8 & 1.78 & 1.9 & 0.1697 & 1 & 0.521 \\
\hline $03-204-15.1 \mathrm{c}$ & 0.000003 & 0.0767 & 0.07 & 752 & 0.23 & 1097.6 & 15.5 & 1111 & 19 & 1 & 1.96 & 1.8 & 0.1857 & 1.5 & 0.833 \\
\hline $03-204-15.2 \mathrm{c}$ & 0.000015 & 0.0853 & 0.83 & 151 & 0.26 & 1153.6 & 45.9 & 1318 & 86 & 12 & 2.32 & 6 & 0.1976 & 4.1 & 0.681 \\
\hline $03-204-16.1 \mathrm{c}$ & 0.000012 & 0.0748 & 0.18 & 86 & 0.33 & 1020.5 & 9.9 & 1057 & 31 & 3 & 1.77 & 1.8 & 0.1718 & 1 & 0.547 \\
\hline
\end{tabular}


Table 2 (Continued)

\begin{tabular}{|c|c|c|c|c|c|c|c|c|c|c|c|c|c|c|c|}
\hline $\begin{array}{l}\begin{array}{l}\text { Sample } \\
\text { (location) }\end{array} \\
\text { (a) }\end{array}$ & $\begin{array}{c}\text { Measured } \\
{ }^{204} \mathrm{~Pb} /{ }^{206} \mathrm{~Pb}\end{array}$ & $\begin{array}{c}\text { Measured } \\
{ }^{207} \mathrm{~Pb} / 206 \\
\mathrm{~Pb}\end{array}$ & $\begin{array}{l}\text { \% Common } \\
{ }^{206} \mathrm{~Pb}\end{array}$ & $\begin{array}{c}\mathrm{U} \\
(\mathrm{ppm})\end{array}$ & $\mathrm{Th} / \mathrm{U}$ & $\begin{array}{c}{ }^{206} \mathrm{~Pb}^{\mathrm{b}} /{ }^{238} \mathrm{U} \\
\text { (Ma) }\end{array}$ & $\begin{array}{l}\mathrm{Err}^{\mathrm{C}} \\
(\mathrm{Ma})\end{array}$ & $\begin{array}{c}{ }^{207} \mathrm{~Pb}^{\mathrm{b}} / 206 \\
\text { (Ma) }\end{array}$ & $\begin{array}{l}\mathrm{Err}^{\mathrm{c}} \\
(\mathrm{Ma})\end{array}$ & $\begin{array}{c}\text { Disc. } \\
\%\end{array}$ & ${ }^{207} \mathrm{~Pb}^{\mathrm{d} / 235} \mathrm{U}$ & $\begin{array}{l}\operatorname{Err}^{\mathrm{c}} \\
(\%)\end{array}$ & ${ }^{206} \mathrm{~Pb}^{\mathrm{d}} /{ }^{238} \mathrm{U}$ & $\begin{array}{l}\operatorname{Err}^{\mathrm{c}} \\
(\%)\end{array}$ & $\begin{array}{l}\text { Err } \\
\text { corr }\end{array}$ \\
\hline $03-204-17.1 \mathrm{c}$ & 0.000053 & 0.0772 & 0.02 & 155 & 0.34 & 1120.3 & 9.9 & 1106 & 24 & -1 & 2 & 1.5 & 0.1897 & 0.9 & 0.601 \\
\hline 03-204-18.1c & 0.000058 & 0.076 & -0.13 & 157 & 0.39 & 1122.2 & 24.2 & 1072 & 23 & -5 & 1.97 & 2.5 & 0.1897 & 2.2 & 0.894 \\
\hline \multicolumn{16}{|c|}{ SNP-02-177 foliated pyroxene monzogranite (farsundite) (Yfpg), Chester Gap quadrangle, UTM coordinates: Easting: 0747 420, Northing: 4293520} \\
\hline $02-177-1.1 \mathrm{c}$ & 0.000041 & 0.0798 & 0.07 & 334 & 0.31 & 1132.5 & 7.8 & 1178 & 17 & 4 & 2.1 & 1.1 & 0.1925 & 0.7 & 0.628 \\
\hline $02-177-2.1 \mathrm{c}$ & -0.000003 & 0.0761 & 0 & 656 & 0.3 & 1074.7 & 6.7 & 1100 & 19 & 2 & 1.91 & 1.2 & 0.1816 & 0.6 & 0.55 \\
\hline $02-177-3.1 \mathrm{c}$ & -0.000005 & 0.0799 & -0.01 & 279 & 0.33 & 1079.6 & 7.7 & 1196 & 26 & 10 & 2.02 & 1.5 & 0.1833 & 0.7 & 0.482 \\
\hline $02-177-4.1 \mathrm{c}$ & 0.000022 & 0.0775 & 0.04 & 594 & 0.31 & 1162.5 & 8.3 & 1125 & 27 & -3 & 2.1 & 1.5 & 0.1973 & 0.7 & 0.479 \\
\hline $02-177-5.1 \mathrm{c}$ & 0.000373 & 0.0848 & 0.62 & 299 & 0.39 & 1128.3 & 7.7 & 1186 & 45 & 5 & 2.1 & 2.4 & 0.1918 & 0.7 & 0.294 \\
\hline $02-177-5.2 \mathrm{c}$ & 0.000015 & 0.0753 & 0.02 & 295 & 0.77 & 1047 & 10.2 & 1071 & 21 & 2 & 1.83 & 1.4 & 0.1765 & 1 & 0.696 \\
\hline $02-177-6.1 \mathrm{c}$ & 0.000026 & 0.0774 & 0.04 & 627 & 0.31 & 1147.6 & 9 & 1122 & 14 & -2 & 2.07 & 1.1 & 0.1946 & 0.8 & 0.764 \\
\hline $02-177-7.1 \mathrm{r}$ & 0.000104 & 0.0752 & 0.18 & 275 & 0.77 & 1055 & 7.6 & 1035 & 21 & -2 & 1.81 & 1.3 & 0.1776 & 0.7 & 0.592 \\
\hline $02-177-7.2 \mathrm{c}$ & 0.000062 & 0.0764 & 0.1 & 220 & 0.35 & 1133.1 & 10.7 & 1083 & 20 & -5 & 2 & 1.4 & 0.1917 & 1 & 0.706 \\
\hline $02-177-8.1 \mathrm{r}$ & 0.00001 & 0.075 & 0.02 & 630 & 0.3 & 1026.6 & 8.1 & 1066 & 12 & 4 & 1.79 & 1 & 0.1729 & 0.8 & 0.8 \\
\hline $02-177-8.2 \mathrm{c}$ & 0.000015 & 0.079 & 0.02 & 455 & 0.46 & 1144 & 7.3 & 1168 & 11 & 2 & 2.11 & 0.9 & 0.1944 & 0.7 & 0.779 \\
\hline $02-177-9.1 \mathrm{c}$ & 0.00003 & 0.0775 & 0.05 & 561 & 0.29 & 1115.8 & 15.2 & 1123 & 31 & 1 & 2.01 & 2.1 & 0.189 & 1.4 & 0.674 \\
\hline $02-177-9.2 \mathrm{c}$ & 0.000053 & 0.078 & 0.09 & 251 & 0.35 & 1046.1 & 7.6 & 1128 & 17 & 7 & 1.88 & 1.2 & 0.1768 & 0.8 & 0.653 \\
\hline $02-177-10.1 \mathrm{c}$ & 0.000013 & 0.0784 & 0.02 & 451 & 0.36 & 1093.8 & 9.3 & 1151 & 22 & 5 & 2 & 1.4 & 0.1854 & 0.9 & 0.623 \\
\hline $02-177-10.2 \mathrm{c}$ & 0.000042 & 0.077 & 0.07 & 354 & 0.32 & 1142.7 & 12 & 1105 & 25 & -3 & 2.04 & 1.6 & 0.1936 & 1.1 & 0.658 \\
\hline $02-177-11.1 \mathrm{c}$ & 0.000061 & 0.0799 & 0.1 & 274 & 0.33 & 1098.3 & 13.7 & 1172 & 34 & 6 & 2.03 & 2.2 & 0.1864 & 1.3 & 0.595 \\
\hline $02-177-11.2 \mathrm{r}$ & 0.000001 & 0.0742 & 0 & 289 & 0.95 & 1014.5 & 7 & 1047 & 17 & 3 & 1.75 & 1.1 & 0.1707 & 0.7 & 0.639 \\
\hline $02-177-12.1 \mathrm{c}$ & 0.000023 & 0.0768 & 0.04 & 699 & 0.31 & 1178 & 9.5 & 1108 & 22 & -6 & 2.11 & 1.4 & 0.1999 & 0.8 & 0.601 \\
\hline $02-177-13.1 \mathrm{c}$ & 0.000018 & 0.0799 & 0.03 & 710 & 0.34 & 1148.7 & 7.2 & 1189 & 14 & 3 & 2.15 & 0.9 & 0.1954 & 0.6 & 0.686 \\
\hline $02-177-14.1 \mathrm{c}$ & 0.000019 & 0.0783 & 0.03 & 687 & 0.24 & 1069.2 & 7 & 1148 & 42 & 7 & 1.95 & 2.2 & 0.181 & 0.7 & 0.292 \\
\hline $02-177-15.1 \mathrm{c}$ & 0.000042 & 0.0778 & 0.07 & 434 & 0.28 & 1087.5 & 13.8 & 1127 & 52 & 4 & 1.96 & 2.9 & 0.1841 & 1.3 & 0.448 \\
\hline $02-177-16.1 \mathrm{c}$ & 0 & 0.0765 & 0 & 484 & 0.28 & 1092.3 & 8.2 & 1109 & 32 & 2 & 1.95 & 1.8 & 0.1848 & 0.8 & 0.425 \\
\hline $02-177-17.1 \mathrm{c}$ & -0.00001 & 0.077 & -0.02 & 934 & 0.29 & 1068.6 & 6.5 & 1125 & 17 & 5 & 1.92 & 1.1 & 0.1808 & 0.6 & 0.592 \\
\hline $02-177-18.1 \mathrm{r}$ & 0.000055 & 0.0736 & 0.09 & 346 & 0.8 & 1028 & 6.8 & 1008 & 14 & -2 & 1.73 & 1 & 0.1727 & 0.7 & 0.7 \\
\hline $02-177-19.1 \mathrm{r}$ & 0.000037 & 0.0743 & 0.06 & 315 & 1.01 & 1018.5 & 6.9 & 1036 & 18 & 2 & 1.74 & 1.1 & 0.1713 & 0.7 & 0.619 \\
\hline $02-177-20.1 \mathrm{r}$ & 0.000022 & 0.0744 & 0.04 & 459 & 0.65 & 1010.8 & 6.5 & 1043 & 13 & 3 & 1.74 & 0.9 & 0.17 & 0.7 & 0.708 \\
\hline \multicolumn{16}{|c|}{ SNP-01-146 Flint Hill Gneiss (Yfh), Chester Gap quadrangle, UTM coordinates: Easting: 0747 760, Northing: 4296630} \\
\hline $01-146-1.1 \mathrm{c}$ & 0.000024 & 0.0769 & -0.11 & 462 & 0.31 & 1141.3 & 8.9 & 1109 & 44 & -3 & 2.04 & 2.3 & 0.1934 & 0.8 & 0.339 \\
\hline $01-146-2.1 \mathrm{c}$ & 0.000005 & 0.0762 & -0.04 & 327 & 0.34 & 1109.7 & 8.9 & 1099 & 14 & -1 & 1.97 & 1.1 & 0.1878 & 0.8 & 0.758 \\
\hline $01-146-2.2 \mathrm{r}$ & 0.000095 & 0.0748 & -0.94 & 266 & 0.5 & 1265.6 & 10.5 & 1027 & 32 & -23 & 2.17 & 1.8 & 0.2146 & 0.9 & 0.48 \\
\hline $01-146-3.1 \mathrm{c}$ & 0.000021 & 0.0794 & 0.36 & 625 & 0.31 & 1105.1 & 8.1 & 1175 & 22 & 6 & 2.05 & 1.3 & 0.1876 & 0.8 & 0.565 \\
\hline $01-146-4.1 \mathrm{c}$ & 0.000034 & 0.0784 & 0.01 & 329 & 0.33 & 1154.8 & 9.4 & 1145 & 25 & -1 & 2.11 & 1.5 & 0.1961 & 0.8 & 0.558 \\
\hline $01-146-4.2 \mathrm{c}$ & 0.000013 & 0.0783 & -0.11 & 511 & 0.63 & 1178 & 8.7 & 1151 & 9 & -2 & 2.16 & 0.9 & 0.2002 & 0.8 & 0.863 \\
\hline $01-146-5.1 \mathrm{c}$ & 0.000004 & 0.0776 & -0.03 & 392 & 0.34 & 1143.1 & 15 & 1135 & 23 & -1 & 2.07 & 1.8 & 0.194 & 1.4 & 0.765 \\
\hline $01-146-5.1 \mathrm{c}$ & 0.000011 & 0.0809 & 0.31 & 597 & 0.35 & 1153.7 & 8.4 & 1216 & 13 & 5 & 2.19 & 1 & 0.1966 & 0.8 & 0.758 \\
\hline $01-146-6.1 \mathrm{c}$ & 0.000013 & 0.0786 & -0.02 & 488 & 0.37 & 1166.9 & 8.9 & 1157 & 14 & -1 & 2.14 & 1.1 & 0.1983 & 0.8 & 0.751 \\
\hline $01-146-8.1 \mathrm{c}$ & 0.000017 & 0.0791 & 0.26 & 427 & 0.33 & 1118.1 & 8.8 & 1168 & 11 & 4 & 2.06 & 1 & 0.1898 & 0.8 & 0.83 \\
\hline $01-146-9.1 \mathrm{c}$ & -0.000001 & 0.0768 & -0.22 & 570 & 0.31 & 1161.9 & 11.8 & 1115 & 23 & -4 & 2.09 & 1.5 & 0.1971 & 1 & 0.677 \\
\hline $01-146-10.1 \mathrm{c}$ & 0.000019 & 0.0781 & 0.09 & 503 & 0.33 & 1129.3 & 8.7 & 1142 & 23 & 1 & 2.06 & 1.4 & 0.1916 & 0.8 & 0.565 \\
\hline $01-146-11.1 \mathrm{c}$ & 0.00001 & 0.0804 & 0.46 & 542 & 0.32 & 1108.1 & 8.5 & 1202 & 21 & 8 & 2.08 & 1.3 & 0.1884 & 0.8 & 0.603 \\
\hline $01-146-12.1 \mathrm{c}$ & -0.000009 & 0.0783 & 0.19 & 382 & 0.32 & 1112.4 & 12.5 & 1157 & 44 & 4 & 2.04 & 2.5 & 0.1887 & 1.1 & 0.463 \\
\hline $01-146-13.1 \mathrm{c}$ & 0.000001 & 0.0772 & 0.25 & 415 & 0.3 & 1071.4 & 8.4 & 1127 & 14 & 5 & 1.93 & 1.1 & 0.1813 & 0.8 & 0.749 \\
\hline $01-146-14.1 \mathrm{c}$ & 0.000055 & 0.0754 & -0.14 & 283 & 0.34 & 1110.9 & 9 & 1059 & 23 & -5 & 1.93 & 1.4 & 0.1876 & 0.8 & 0.595 \\
\hline $01-146-15.1 \mathrm{c}$ & 0.00006 & 0.0746 & 0.24 & 289 & 0.37 & 1001.6 & 8.1 & 1034 & 15 & 3 & 1.71 & 1.1 & 0.1683 & 0.8 & 0.756 \\
\hline $01-146-16.1 \mathrm{c}$ & 0.000018 & 0.076 & -0.18 & 513 & 0.34 & 1132.9 & 8.8 & 1087 & 35 & -4 & 2 & 1.9 & 0.1917 & 0.8 & 0.416 \\
\hline $01-146-17.1 \mathrm{r}$ & 0.000374 & 0.0829 & 0.81 & 1489 & 0.29 & 1096.8 & 7.7 & 1137 & 22 & 4 & 1.99 & 1.3 & 0.1858 & 0.7 & 0.545 \\
\hline $01-146-17.2 \mathrm{c}$ & 0.000005 & 0.0762 & -0.11 & 590 & 0.33 & 1124.4 & 12.8 & 1098 & 17 & -2 & 2 & 1.5 & 0.1903 & 1.2 & 0.814 \\
\hline $01-146-18.1 \mathrm{r}$ & 0.000027 & 0.0784 & 0.08 & 1050 & 0.21 & 1141.3 & 8.3 & 1148 & 16 & 1 & 2.09 & 1.1 & 0.1937 & 0.7 & 0.691 \\
\hline $01-146-18.2 \mathrm{c}$ & -0.000002 & 0.0808 & 0.7 & 366 & 0.34 & 1065.6 & 12.8 & 1216 & 10 & 12 & 2.02 & 1.4 & 0.181 & 1.2 & 0.921 \\
\hline $01-146-19.1 \mathrm{r}$ & 0.000074 & 0.0793 & 0.85 & 1766 & 2.02 & 990.2 & 7.6 & 1153 & 14 & 14 & 1.8 & 1.1 & 0.1672 & 0.8 & 0.743 \\
\hline $01-146-19.2 \mathrm{c}$ & 0.000021 & 0.0775 & 0.24 & 396 & 0.33 & 1080.8 & 15.4 & 1125 & 16 & 4 & 1.95 & 1.7 & 0.1829 & 1.5 & 0.878 \\
\hline
\end{tabular}


SNP-01-175 pyroxene quartz diorite (orthopyroxene quartz diorite) (Ypqd), Chester Gap quadrangle, UTM coordinates: Easting: 0740 412, Northing: 4302664

\begin{tabular}{|c|c|c|c|c|c|c|c|c|}
\hline $01-175-1.1 \mathrm{c}$ & 0.000013 & 0.076 & -0.04 & 162 & 0.75 & 1101.8 & 9.7 & 1089 \\
\hline $01-175-2.1 \mathrm{c}$ & 0.000073 & 0.0748 & 0.13 & 95 & 0.47 & 1033.3 & 12.1 & 1035 \\
\hline $01-175-3.1 \mathrm{c}$ & -0.000005 & 0.0783 & 0.02 & 205 & 0.76 & 1151.2 & 13.6 & 1157 \\
\hline $01-175-4.1 \mathrm{c}$ & -0.000001 & 0.0801 & 0.17 & 158 & 0.75 & 1164.7 & 20.3 & 1200 \\
\hline $01-175-5.1 \mathrm{c}$ & -0.000039 & 0.0789 & -0.01 & 169 & 0.76 & 1171.7 & 11.1 & 1183 \\
\hline $01-175-6.1 \mathrm{c}$ & -0.000041 & 0.0724 & -0.06 & 56 & 0.42 & 1011.7 & 10.9 & 1013 \\
\hline $01-175-7.1 \mathrm{c}$ & 0.000109 & 0.0813 & 0.2 & 109 & 0.78 & 1188.8 & 10.8 & 1192 \\
\hline $01-175-8.1 \mathrm{c}$ & 0.000032 & 0.0782 & 0.02 & 141 & 0.63 & 1148.1 & 13.8 & 1141 \\
\hline $01-175-8.2 \mathrm{c}$ & -0.000029 & 0.0787 & 0.09 & 170 & 0.4 & 1144.4 & 11.9 & 1175 \\
\hline $01-175-9.1 \mathrm{c}$ & 0 & 0.08 & 0.43 & 119 & 0.74 & 1107 & 10 & 1198 \\
\hline $01-175-10.1 \mathrm{c}$ & 0.000089 & 0.0772 & -0.01 & 88 & 0.6 & 1128.7 & 16.8 & 1093 \\
\hline $01-175-10.2 \mathrm{r}$ & 0.001779 & 0.0768 & 0.25 & 17 & 0.39 & 1060 & 17.9 & 231 \\
\hline $01-175-11.1 \mathrm{c}$ & 0.000043 & 0.0757 & 0 & 100 & 0.58 & 1086.4 & 10.3 & 1071 \\
\hline $01-175-12.1 \mathrm{c}$ & 0.000038 & 0.082 & -0.02 & 120 & 0.71 & 1250 & 11.3 & 1233 \\
\hline $01-175-13.1 \mathrm{c}$ & 0.000037 & 0.0821 & 0.01 & 146 & 0.77 & 1245.8 & 30 & 1236 \\
\hline $01-175-14.1 \mathrm{c}$ & 0.000051 & 0.0786 & 0.06 & 120 & 0.74 & 1148.8 & 15.5 & 1144 \\
\hline $01-175-15.1 \mathrm{c}$ & -0.000009 & 0.0843 & 0.04 & 149 & 0.75 & 1291 & 18.8 & 1302 \\
\hline $01-175-16.1 \mathrm{c}$ & 0.000007 & 0.0765 & -0.05 & 145 & 0.62 & 1118.8 & 10.2 & 1106 \\
\hline $01-175-17.1 \mathrm{c}$ & 0.000074 & 0.0794 & 0.24 & 133 & 0.76 & 1131.3 & 13.5 & 1156 \\
\hline $01-175-18.1 \mathrm{c}$ & 0.000052 & 0.0785 & 0 & 170 & 0.76 & 1159.9 & 9.7 & 1141 \\
\hline $01-175-19.1 \mathrm{c}$ & 0.000101 & 0.0735 & 0.06 & 91 & 0.66 & 1016 & 10.2 & 989 \\
\hline $01-175-20.1 \mathrm{c}$ & 0.000105 & 0.0726 & -0.08 & 88 & 0.61 & 1020.9 & 15 & 960 \\
\hline $01-175-20.2 \mathrm{r}$ & 0.000222 & 0.0711 & -0.07 & 27 & 0.84 & 978.2 & 13.7 & 867 \\
\hline \multicolumn{9}{|c|}{ SNP-96-1 garnetiferous granite (charnockite to farsundite) gneiss (Yg), Thornton Gap quadrangle, UTM coordinates: Easting: 0734 425, Northing: 4281017} \\
\hline $96-1-1 \mathrm{Z}-1.1 \mathrm{c}$ & 0.00174 & 0.1039 & 2.91 & 195 & 0.41 & 1180.5 & 10.8 & 1179 \\
\hline $96-1-1 Z-1.2 \mathrm{c}$ & 0.000629 & 0.0856 & 1.06 & 210 & 0.4 & 1155.9 & 10.3 & 1112 \\
\hline 96-1-1Z-2.1c & 0.000053 & 0.0768 & 0.09 & 366 & 0.44 & 1138.8 & 7.6 & 1096 \\
\hline $96-1-1 Z-2.2 c$ & 0.001334 & 0.0939 & 2.26 & 386 & 0.35 & 1156.8 & 8.4 & 1068 \\
\hline $96-1-1 \mathrm{Z}-3.1 \mathrm{r}$ & 0.000016 & 0.0737 & 0.03 & 1447 & 0.07 & 1090.5 & 4 & 1026 \\
\hline $96-1-1 Z-3.2 \mathrm{r}$ & 0.000058 & 0.0746 & 0.1 & 593 & 0.63 & 1069.4 & 6.3 & 1035 \\
\hline $96-1-1 \mathrm{Z}-4.1 \mathrm{c}$ & 0.000086 & 0.0807 & 0.14 & 178 & 0.39 & 1220.3 & 12.9 & 1185 \\
\hline $96-1-1 Z-5.1 \mathrm{c}$ & 0.000238 & 0.0754 & 0.41 & 134 & 0.3 & 1068.7 & 11.8 & 987 \\
\hline $96-1-1 Z-6.1 \mathrm{c}$ & 0.000088 & 0.0771 & 0.15 & 194 & 0.33 & 1108.3 & 10.1 & 1091 \\
\hline $96-1-1 \mathrm{Z}-7.1 \mathrm{c}$ & 0.000144 & 0.0814 & 0.24 & 168 & 0.75 & 1209.5 & 13.1 & 1182 \\
\hline $96-1-1 Z-8.1 \mathrm{c}$ & 0.00007 & 0.0804 & 0.12 & 309 & 0.36 & 1185.5 & 8.5 & 1183 \\
\hline $96-1-1 \mathrm{Z}-9.1 \mathrm{c}$ & 0.000158 & 0.0806 & 0.27 & 407 & 0.18 & 1079 & 7.7 & 1155 \\
\hline $96-1-1 \mathrm{Z}-10.1 \mathrm{c}$ & 0.000054 & 0.0796 & 0.09 & 468 & 0.32 & 1175.4 & 10.6 & 1168 \\
\hline $96-1-1 \mathrm{Z}-11.1 \mathrm{c}$ & 0.000059 & 0.08 & 0.1 & 561 & 0.19 & 1263.8 & 7.7 & 1175 \\
\hline $96-1-1 \mathrm{Z}-11.2 \mathrm{r}$ & 0.000046 & 0.074 & 0.08 & 528 & 0.51 & 1053.1 & 6 & 1024 \\
\hline $96-1-1 \mathrm{Z}-12.1 \mathrm{c}$ & 0.000014 & 0.0813 & 0.02 & 413 & 0.37 & 1239.6 & 7.8 & 1223 \\
\hline $96-1-1 \mathrm{Z}-13.1 \mathrm{c}$ & 0.00009 & 0.0801 & 0.15 & 393 & 0.27 & 1183.8 & 8.5 & 1167 \\
\hline $96-1-1 \mathrm{Z}-14.1 \mathrm{c}$ & 0.000147 & 0.0807 & 0.25 & 242 & 0.38 & 1188.3 & 9.8 & 1163 \\
\hline $96-1-1 Z-15.1 \mathrm{c}$ & -0.000005 & 0.0853 & -0.01 & 315 & 0.27 & 1385.7 & 9.7 & 1324 \\
\hline 96-1-1Z-16.1c & 0.000088 & 0.0796 & 0.15 & 246 & 0.37 & 1110.4 & 10.3 & 1157 \\
\hline $96-1-1 \mathrm{Z}-17.1 \mathrm{r}$ & 0.000086 & 0.0742 & 0.15 & 361 & 0.65 & 1044.3 & 7.3 & 1014 \\
\hline $96-1-1 Z-17.2 \mathrm{c}$ & 0.000034 & 0.0804 & 0.06 & 218 & 0.65 & 1147 & 9.9 & 1194 \\
\hline $96-1-1 Z-18.1 \mathrm{c}$ & 0.000196 & 0.0801 & 0.33 & 579 & 0.3 & 1092.1 & 6.6 & 1131 \\
\hline 96-1-1Z-19.1c & 0.000162 & 0.0793 & 0.27 & 148 & 0.35 & 1083.3 & 23.7 & 1122 \\
\hline $96-1-1 \mathrm{Z}-20.1 \mathrm{c}$ & 0.000153 & 0.0796 & 0.26 & 210 & 0.36 & 1139.7 & 10.1 & 1132 \\
\hline $96-1-1 \mathrm{Z}-21.1 \mathrm{c}$ & 0.000036 & 0.0783 & 0.06 & 262 & 0.67 & 1182.3 & 9.3 & 1141 \\
\hline
\end{tabular}

\begin{tabular}{|c|c|c|c|c|c|}
\hline-1 & 1.95 & 1.4 & 0.1863 & 0.9 & 0.643 \\
\hline 0 & 1.77 & 2 & 0.1739 & 1.2 & 0.605 \\
\hline 1 & 2.11 & 1.4 & 0.1956 & 1.2 & 0.906 \\
\hline 3 & 2.19 & 1.9 & 0.1984 & 1.8 & 0.94 \\
\hline 1 & 2.18 & 1.2 & 0.1994 & 1 & 0.838 \\
\hline 0 & 1.71 & 1.6 & 0.1699 & 1.1 & 0.679 \\
\hline 0 & 2.23 & 1.4 & 0.2025 & 0.9 & 0.686 \\
\hline-1 & 2.09 & 1.5 & 0.1949 & 1.2 & 0.846 \\
\hline 3 & 2.12 & 3 & 0.1945 & 1.1 & 0.351 \\
\hline 8 & 2.08 & 1.5 & 0.1881 & 0.9 & 0.611 \\
\hline-3 & 2 & 2.5 & 0.191 & 1.5 & 0.602 \\
\hline-359 & 1.22 & 15 & 0.1738 & 1.9 & 0.127 \\
\hline-1 & 1.9 & 1.7 & 0.1834 & 1 & 0.581 \\
\hline-1 & 2.4 & 1.5 & 0.2138 & 0.9 & 0.618 \\
\hline-1 & 2.4 & 2.9 & 0.2131 & 2.5 & 0.851 \\
\hline 0 & 2.09 & 1.7 & 0.195 & 1.4 & 0.846 \\
\hline 1 & 2.58 & 1.7 & 0.2218 & 1.5 & 0.895 \\
\hline-1 & 2 & 1.2 & 0.1894 & 0.9 & 0.758 \\
\hline 2 & 2.08 & 1.6 & 0.1921 & 1.2 & 0.779 \\
\hline-2 & 2.11 & 1.2 & 0.197 & 0.9 & 0.736 \\
\hline-3 & 1.69 & 1.8 & 0.1705 & 1 & 0.566 \\
\hline-6 & 1.68 & 2.1 & 0.1711 & 1.5 & 0.729 \\
\hline-13 & 1.53 & 5.9 & 0.1631 & 1.5 & 0.248 \\
\hline 0 & 2.2 & 6.9 & 0.2009 & 1.1 & 0.16 \\
\hline-4 & 2.07 & 3.3 & 0.196 & 0.9 & 0.277 \\
\hline-4 & 2.02 & 1.1 & 0.1928 & 0.7 & 0.608 \\
\hline-8 & 2.02 & 3.3 & 0.1958 & 0.8 & 0.239 \\
\hline-6 & 1.86 & 0.7 & 0.1838 & 0.4 & 0.584 \\
\hline-3 & 1.83 & 1.1 & 0.1802 & 0.6 & 0.561 \\
\hline-3 & 2.28 & 2.5 & 0.208 & 1.1 & 0.433 \\
\hline-8 & 1.78 & 2.5 & 0.1797 & 1.1 & 0.453 \\
\hline-2 & 1.96 & 1.6 & 0.1874 & 0.9 & 0.58 \\
\hline-2 & 2.26 & 1.9 & 0.2061 & 1.1 & 0.597 \\
\hline 0 & 2.21 & 1.3 & 0.2019 & 0.7 & 0.588 \\
\hline 7 & 1.97 & 1.8 & 0.1828 & 0.7 & 0.418 \\
\hline-1 & 2.17 & 1.2 & 0.1999 & 0.9 & 0.77 \\
\hline-8 & 2.35 & 1.5 & 0.2157 & 0.6 & 0.416 \\
\hline-3 & 1.79 & 0.9 & 0.1772 & 0.6 & 0.636 \\
\hline-1 & 2.37 & 1.1 & 0.2119 & 0.6 & 0.576 \\
\hline-1 & 2.19 & 1.3 & 0.2014 & 0.7 & 0.567 \\
\hline-2 & 2.19 & 1.9 & 0.2022 & 0.9 & 0.44 \\
\hline-5 & 2.81 & 1 & 0.239 & 0.7 & 0.716 \\
\hline 4 & 2.04 & 2.1 & 0.1884 & 0.9 & 0.455 \\
\hline-3 & 1.77 & 1.4 & 0.1756 & 0.7 & 0.523 \\
\hline 4 & 2.15 & 1.5 & 0.1952 & 0.9 & 0.588 \\
\hline 3 & 1.97 & 1.5 & 0.1849 & 0.6 & 0.405 \\
\hline 3 & 1.95 & 2.8 & 0.1833 & 2.3 & 0.816 \\
\hline-1 & 2.06 & 1.7 & 0.1933 & 0.9 & 0.555 \\
\hline-4 & 2.15 & 1.3 & 0.2009 & 0.8 & 0.642 \\
\hline
\end{tabular}


Table 2 (Continued)

\begin{tabular}{|c|c|c|c|c|c|c|c|c|c|c|c|c|c|c|c|}
\hline $\begin{array}{l}\text { Sample }{ }^{\mathrm{a}} \\
\text { (location) }\end{array}$ & $\begin{array}{c}\text { Measured } \\
{ }^{204} \mathrm{~Pb} /{ }^{206} \mathrm{~Pb}\end{array}$ & $\begin{array}{c}\text { Measured } \\
{ }^{207} \mathrm{~Pb} /{ }^{206} \mathrm{~Pb}\end{array}$ & $\begin{array}{l}\text { \% Common } \\
{ }^{206} \mathrm{~Pb}\end{array}$ & $\begin{array}{c}\mathrm{U} \\
(\mathrm{ppm})\end{array}$ & $\mathrm{Th} / \mathrm{U}$ & $\begin{array}{c}{ }^{206} \mathrm{~Pb}^{\mathrm{b} /} /^{238} \mathrm{U} \\
(\mathrm{Ma})\end{array}$ & $\begin{array}{l}\text { Err }^{\mathrm{c}} \\
(\mathrm{Ma})\end{array}$ & $\begin{array}{c}{ }^{207} \mathrm{~Pb}^{\mathrm{b}} / 206 \\
\text { (Ma) }\end{array}$ & $\begin{array}{l}\mathrm{Err}^{\mathrm{c}} \\
(\mathrm{Ma})\end{array}$ & $\begin{array}{c}\text { Disc. } \\
\%\end{array}$ & ${ }^{207} \mathrm{~Pb}^{\mathrm{d} / 235} \mathrm{U}$ & $\begin{array}{l}\operatorname{Err}^{\mathrm{c}} \\
(\%)\end{array}$ & ${ }^{206} \mathrm{~Pb}^{\mathrm{d} / 238} \mathrm{U}$ & $\begin{array}{l}\operatorname{Err}^{\mathrm{c}} \\
(\%)\end{array}$ & $\begin{array}{l}\text { Err } \\
\text { corr }\end{array}$ \\
\hline $96-1-1 \mathrm{Z}-22.1 \mathrm{c}$ & 0.000164 & 0.0796 & 0.28 & 117 & 0.51 & 1160 & 13.9 & 1127 & 35 & -3 & 2.1 & 2.1 & 0.1968 & 1.2 & 0.582 \\
\hline $96-1-1 \mathrm{Z}-23.1 \mathrm{c}$ & 0.000224 & 0.0795 & 0.38 & 205 & 0.34 & 1116.7 & 11.3 & 1103 & 38 & -1 & 1.99 & 2.2 & 0.189 & 1.1 & 0.483 \\
\hline $96-1-1 Z-24.1 \mathrm{c}$ & 0.000015 & 0.0788 & 0.02 & 154 & 0.42 & 1080.5 & 11.4 & 1162 & 23 & 7 & 1.99 & 1.6 & 0.1832 & 1.1 & 0.681 \\
\hline \multicolumn{16}{|c|}{ SNP-03-198 garnetiferous granite (charnockite to farsundite) gneiss (Yg), Thornton Gap quadrangle, UTM coordinates: Easting: 0734711 , Northing: 4282659} \\
\hline 03-198-1.1r & 0.000003 & 0.0736 & -0.05 & 244 & 1.56 & 1042.7 & 8.8 & 1031 & 27 & -1 & 1.78 & 1.6 & 0.1755 & 0.9 & 0.539 \\
\hline 03-198-2.1r & 0.000054 & 0.0768 & 0.34 & 251 & 1.69 & 1040.8 & 8.5 & 1096 & 17 & 5 & 1.84 & 1.2 & 0.1757 & 0.8 & 0.713 \\
\hline 03-198-3.1r & -0.000012 & 0.075 & 0.07 & 260 & 1.8 & 1053.7 & 8.8 & 1074 & 24 & 2 & 1.84 & 1.5 & 0.1777 & 0.9 & 0.579 \\
\hline 03-198-4.1c & 0.000026 & 0.0741 & -0.13 & 707 & 0.14 & 1072.9 & 7.9 & 1033 & 9 & -4 & 1.84 & 0.9 & 0.1808 & 0.8 & 0.873 \\
\hline $03-198-5.1 \mathrm{c}$ & 0.000005 & 0.0768 & 0.16 & 400 & 0.09 & 1080.2 & 8.6 & 1113 & 24 & 3 & 1.93 & 1.5 & 0.1827 & 0.8 & 0.557 \\
\hline 03-198-6.1r & 0.000001 & 0.075 & 0.14 & 240 & 1.49 & 1038.1 & 8.7 & 1069 & 17 & 3 & 1.81 & 1.2 & 0.175 & 0.9 & 0.711 \\
\hline $03-198-6.2 \mathrm{c}$ & -0.000006 & 0.0761 & 0.12 & 565 & 0.34 & 1069.5 & 8 & 1099 & 26 & 3 & 1.9 & 1.5 & 0.1807 & 0.8 & 0.503 \\
\hline 03-198-7.1r & 0.000063 & 0.0755 & 0.14 & 308 & 1.39 & 1049.8 & 8.4 & 1058 & 25 & 1 & 1.82 & 1.5 & 0.1769 & 0.8 & 0.549 \\
\hline $03-198-8.1 \mathrm{c}$ & -0.000004 & 0.0825 & 0.57 & 344 & 0.17 & 1141.4 & 17.9 & 1260 & 61 & 9 & 2.22 & 3.5 & 0.1948 & 1.6 & 0.454 \\
\hline $03-198-10.1 \mathrm{c}$ & 0.000016 & 0.0817 & 0.38 & 520 & 0.45 & 1158.1 & 10.2 & 1232 & 37 & 6 & 2.22 & 2.1 & 0.1975 & 0.9 & 0.425 \\
\hline $03-198-11.1 \mathrm{c}$ & -0.000006 & 0.0758 & -0.24 & 278 & 1.62 & 1142.4 & 9.3 & 1092 & 12 & -5 & 2.02 & 1 & 0.1935 & 0.8 & 0.814 \\
\hline $03-198-9.2 \mathrm{c}$ & 0.000014 & 0.076 & -0.7 & 325 & 0.15 & 1245.3 & 24.4 & 1091 & 41 & -14 & 2.21 & 2.9 & 0.2116 & 2 & 0.699 \\
\hline $03-198-12.1 \mathrm{c}$ & 0.000025 & 0.0726 & -0.14 & 462 & 0.3 & 1034.2 & 7.7 & 993 & 21 & -4 & 1.73 & 1.3 & 0.1737 & 0.8 & 0.588 \\
\hline $03-198-12.2 \mathrm{r}$ & 0.000013 & 0.073 & -0.11 & 743 & 0.2 & 1038.6 & 7.4 & 1009 & 10 & -3 & 1.75 & 0.9 & 0.1746 & 0.7 & 0.82 \\
\hline $03-198-13.1 \mathrm{c}$ & 0.000105 & 0.0812 & 0.18 & 218 & 0.59 & 1190.3 & 12.2 & 1191 & 17 & 0 & 2.23 & 1.4 & 0.2028 & 1.1 & 0.771 \\
\hline 03-198-13.1r & -0.000019 & 0.0777 & 0.15 & 265 & 1.55 & 1104.6 & 9.2 & 1145 & 28 & 4 & 2.01 & 1.7 & 0.1873 & 0.9 & 0.519 \\
\hline $03-198-14.1 \mathrm{c}$ & 0.000042 & 0.0788 & -0.21 & 295 & 0.47 & 1213.3 & 9.5 & 1153 & 15 & -5 & 2.23 & 1.1 & 0.2065 & 0.8 & 0.722 \\
\hline $03-198-14.2 \mathrm{r}$ & 0.000001 & 0.073 & -0.23 & 308 & 0.92 & 1067.4 & 11.1 & 1014 & 15 & -5 & 1.81 & 1.3 & 0.1797 & 1.1 & 0.83 \\
\hline $03-198-15.1 \mathrm{c}$ & 0.000042 & 0.076 & -0.19 & 161 & 0.57 & 1138.5 & 10.6 & 1080 & 18 & -5 & 2 & 1.3 & 0.1927 & 1 & 0.726 \\
\hline $03-198-15.2 \mathrm{r}$ & 0.000071 & 0.0727 & -0.1 & 248 & 1.37 & 1028.7 & 8.4 & 977 & 16 & -5 & 1.71 & 1.1 & 0.1726 & 0.8 & 0.738 \\
\hline $03-198-16.1 \mathrm{c}$ & 0.000057 & 0.0767 & -0.26 & 178 & 0.29 & 1169.1 & 10.3 & 1093 & 48 & -7 & 2.07 & 2.5 & 0.1981 & 0.9 & 0.352 \\
\hline 03-198-17.1c & 0.000012 & 0.081 & -0.42 & 507 & 0.4 & 1304.2 & 13.1 & 1216 & 17 & -7 & 2.49 & 1.3 & 0.2233 & 1 & 0.772 \\
\hline \multicolumn{16}{|c|}{ SNP-01-150 layered granodiorite (opdalite) gneiss (Ylgn), Chester Gap quadrangle, UTM coordinates: Easting: 0744 658, Northing: 4301678} \\
\hline $01-150-1.1 \mathrm{c}$ & 0.000006 & 0.0789 & 0.19 & 253 & 0.44 & 1131.2 & 19.7 & 1169 & 14 & 3 & 2.09 & 1.9 & 0.1921 & 1.8 & 0.931 \\
\hline $01-150-2.1 \mathrm{c}$ & 0.000047 & 0.0801 & 0.28 & 175 & 0.37 & 1138.7 & 10.7 & 1182 & 14 & 4 & 2.12 & 1.2 & 0.1936 & 1 & 0.807 \\
\hline $01-150-3.1 \mathrm{c}$ & 0.000009 & 0.0784 & 0.07 & 543 & 0.49 & 1142.9 & 26.8 & 1155 & 8 & 1 & 2.1 & 2.5 & 0.1941 & 2.4 & 0.986 \\
\hline $01-150-4.1 \mathrm{c}$ & 0.000022 & 0.0781 & -0.02 & 204 & 0.43 & 1154.7 & 10.3 & 1142 & 14 & -1 & 2.1 & 1.2 & 0.1961 & 0.9 & 0.799 \\
\hline $01-150-5.1 \mathrm{c}$ & 0.000019 & 0.0789 & 0.06 & 346 & 0.49 & 1156.1 & 9.1 & 1162 & 9 & 1 & 2.13 & 0.9 & 0.1965 & 0.8 & 0.866 \\
\hline $01-150-6.1 \mathrm{c}$ & 0.000027 & 0.0783 & 0.08 & 217 & 0.44 & 1136.2 & 13.8 & 1144 & 17 & 1 & 2.07 & 1.5 & 0.1928 & 1.3 & 0.823 \\
\hline $01-150-7.1 \mathrm{c}$ & 0.000032 & 0.0788 & -0.02 & 172 & 0.52 & 1170.4 & 9.8 & 1155 & 15 & -1 & 2.15 & 1.2 & 0.1989 & 0.9 & 0.747 \\
\hline $01-150-8.1 \mathrm{c}$ & 0.000039 & 0.0799 & 0.55 & 211 & 0.29 & 1076.5 & 9.2 & 1182 & 15 & 9 & 2 & 1.2 & 0.1826 & 0.9 & 0.766 \\
\hline $01-150-9.1 \mathrm{r}$ & 0.000022 & 0.0753 & 0.02 & 1125 & 0.18 & 1071.1 & 7.8 & 1067 & 10 & 0 & 1.87 & 0.9 & 0.1807 & 0.8 & 0.846 \\
\hline $01-150-10.1 \mathrm{c}$ & -0.000045 & 0.0768 & 0.01 & 116 & 0.34 & 1113.3 & 10.4 & 1132 & 29 & 2 & 2.01 & 1.7 & 0.1887 & 1 & 0.554 \\
\hline $01-150-11.1 \mathrm{c}$ & 0.000127 & 0.077 & -0.04 & 135 & 0.38 & 1129.9 & 11.9 & 1073 & 23 & -5 & 1.98 & 1.6 & 0.1911 & 1.1 & 0.692 \\
\hline $01-150-12.1 \mathrm{c}$ & 0.000053 & 0.0807 & 0.25 & 191 & 0.41 & 1160 & 10.4 & 1195 & 22 & 3 & 2.18 & 1.4 & 0.1975 & 0.9 & 0.641 \\
\hline $01-150-13.1 \mathrm{c}$ & 0.000022 & 0.0787 & 0.13 & 127 & 0.33 & 1137.6 & 10.1 & 1158 & 18 & 2 & 2.09 & 1.3 & 0.1932 & 0.9 & 0.716 \\
\hline $01-150-14.1 \mathrm{c}$ & 0.000036 & 0.0793 & -0.15 & 397 & 0.3 & 1212.1 & 9.8 & 1167 & 20 & -4 & 2.24 & 1.3 & 0.2064 & 0.8 & 0.638 \\
\hline $01-150-15.1 \mathrm{c}$ & 0.000023 & 0.0795 & -0.22 & 190 & 0.37 & 1228.8 & 11.8 & 1175 & 33 & -5 & 2.29 & 1.9 & 0.2095 & 1 & 0.507 \\
\hline $01-150-16.1 \mathrm{c}$ & -0.000006 & 0.0794 & -0.12 & 298 & 0.42 & 1205.5 & 9.5 & 1183 & 19 & -2 & 2.25 & 1.2 & 0.2054 & 0.8 & 0.653 \\
\hline $01-150-17.1 \mathrm{c}$ & 0.000037 & 0.08 & 0.28 & 188 & 0.35 & 1138.6 & 9.5 & 1185 & 24 & 4 & 2.12 & 1.5 & 0.1936 & 0.9 & 0.584 \\
\hline $01-150-18.1 \mathrm{c}$ & -0.000005 & 0.0793 & -0.24 & 270 & 0.43 & 1228.6 & 45.6 & 1181 & 32 & -4 & 2.29 & 4.2 & 0.2095 & 3.9 & 0.922 \\
\hline $01-150-19.1 \mathrm{c}$ & 0.00004 & 0.08 & 0.06 & 230 & 0.42 & 1184.8 & 17 & 1184 & 14 & 0 & 2.21 & 1.7 & 0.2017 & 1.5 & 0.9 \\
\hline $01-150-20.1 \mathrm{c}$ & 0.000011 & 0.0822 & 0.01 & 186 & 0.39 & 1246.2 & 49.7 & 1246 & 22 & 0 & 2.41 & 4.3 & 0.2133 & 4.1 & 0.965 \\
\hline \multicolumn{16}{|c|}{ SNP-03-199 biotite-bearing leucocratic granitoid (Ylg2), Madison quadrangle, UTM coordinates: Easting: 0733 654, Northing: 4256241} \\
\hline 03-199-1.1c & 0.000014 & 0.0794 & 0.38 & 737 & 0.24 & 1101.1 & 17.6 & 1177 & 38 & 6 & 2.04 & 2.5 & 0.1869 & 1.7 & 0.653 \\
\hline 03-199-2.1c & 0.000118 & 0.0792 & 0.19 & 1406 & 0.24 & 1136.2 & 7.9 & 1134 & 24 & 0 & 2.06 & 1.4 & 0.1927 & 0.7 & 0.51 \\
\hline 03-199-3.1c & 0.000911 & 0.092 & 2.83 & 408 & 0.3 & 881.9 & 12.4 & 1174 & 49 & 25 & 1.62 & 2.9 & 0.1485 & 1.4 & 0.506 \\
\hline 03-199-4.1c & 0.000003 & 0.0799 & -0.12 & 761 & 0.34 & 1219 & 16.4 & 1193 & 16 & -2 & 2.29 & 1.6 & 0.2079 & 1.4 & 0.863 \\
\hline 03-199-5.1r & 0.000214 & 0.0777 & 0.36 & 3740 & 0.04 & 1057.8 & 7.3 & 1058 & 11 & 0 & 1.83 & 0.9 & 0.1783 & 0.7 & 0.796 \\
\hline 03-199-6.1r & 0.000003 & 0.0731 & -0.07 & 5498 & 0.06 & 1033.6 & 7.1 & 1017 & 3 & -2 & 1.75 & 0.7 & 0.1738 & 0.7 & 0.98 \\
\hline
\end{tabular}




\begin{tabular}{|c|c|c|c|c|c|c|c|c|c|c|c|c|c|c|c|}
\hline $03-199-6.2 \mathrm{r}$ & 0.001297 & 0.0924 & 1.85 & 3033 & 0.04 & 1117.1 & 12.2 & 1039 & 117 & -8 & 1.92 & 5.9 & 0.1885 & 1.1 & 0.195 \\
\hline $03-199-7.1 \mathrm{c}$ & -0.000006 & 0.0789 & 0.11 & 717 & 0.27 & 1145.6 & 8.4 & 1172 & 7 & 2 & 2.12 & 0.8 & 0.1947 & 0.8 & 0.902 \\
\hline 03-199-8.1r & 0.000022 & 0.074 & -0.03 & 903 & 0.4 & 1049.8 & 7.4 & 1034 & 11 & -2 & 1.8 & 0.9 & 0.1767 & 0.7 & 0.814 \\
\hline $03-199-9.1 \mathrm{c}$ & 0.000001 & 0.0775 & -0.44 & 1412 & 0.76 & 1227.3 & 8.6 & 1135 & 5 & -8 & 2.23 & 0.8 & 0.2088 & 0.7 & 0.943 \\
\hline $03-199-10.1 \mathrm{c}$ & 0.000003 & 0.0776 & -0.11 & 683 & 0.37 & 1160.7 & 8.5 & 1135 & 7 & -2 & 2.11 & 0.8 & 0.197 & 0.8 & 0.897 \\
\hline $03-199-11.1 \mathrm{c}$ & 0.000014 & 0.0789 & 0.09 & 740 & 0.33 & 1149.9 & 8.3 & 1165 & 28 & 1 & 2.12 & 1.6 & 0.1954 & 0.7 & 0.462 \\
\hline $03-199-12.1 \mathrm{r}$ & 0.000017 & 0.0742 & -0.08 & 941 & 0.4 & 1063 & 7.5 & 1039 & 9 & -2 & 1.83 & 0.9 & 0.1791 & 0.7 & 0.847 \\
\hline $03-199-13.1 \mathrm{c}$ & 0.000979 & 0.0896 & 0.52 & 518 & 0.27 & 1320.6 & 10.9 & 1089 & 92 & -21 & 2.35 & 4.7 & 0.2248 & 0.8 & 0.174 \\
\hline $03-199-14.1 \mathrm{c}$ & -0.000001 & 0.079 & 0.1 & 565 & 0.32 & 1149.7 & 9.9 & 1171 & 15 & 2 & 2.13 & 1.2 & 0.1954 & 0.9 & 0.756 \\
\hline 03-199-15.1r & 0.000012 & 0.077 & -0.19 & 912 & 0.27 & 1162.8 & 8.3 & 1117 & 9 & -4 & 2.09 & 0.9 & 0.1973 & 0.7 & 0.85 \\
\hline $03-199-16.1 \mathrm{c}$ & 0.000016 & 0.0775 & -0.15 & 579 & 0.34 & 1166.5 & 11.6 & 1129 & 20 & -3 & 2.11 & 1.4 & 0.198 & 1 & 0.721 \\
\hline 03-199-17.1r & 0.000011 & 0.075 & 0.02 & 891 & 0.41 & 1062.5 & 7.5 & 1064 & 13 & 0 & 1.85 & 1 & 0.1792 & 0.7 & 0.761 \\
\hline $03-199-18.1 \mathrm{c}$ & 0.000655 & 0.0858 & 2.01 & 585 & 1.03 & 900.8 & 6.6 & 1107 & 24 & 19 & 1.6 & 1.4 & 0.1513 & 0.8 & 0.531 \\
\hline 03-199-19.1r & 0.000317 & 0.079 & 0.66 & 417 & 0.4 & 1028.7 & 8.2 & 1056 & 34 & 3 & 1.78 & 1.9 & 0.1732 & 0.8 & 0.43 \\
\hline \multirow{2}{*}{\multicolumn{16}{|c|}{ SNP-02-189 foliated megacrystic leucocratic granite (Ylg1), Madison quadrangle, UTM coordinates: Easting: 0733 654, Northing: 4256241}} \\
\hline & & & 0.08 & 196 & 0.34 & 1099.4 & 16.8 & 1195 & 60 & 8 & 2.06 & 3.4 & 0.1868 & 1.6 & 0.454 \\
\hline $02-189-2.1 \mathrm{c}$ & 0.000032 & 0.0779 & 0.05 & 318 & 0.34 & 1187.3 & 15.8 & 1134 & 33 & -5 & 2.16 & 2.1 & 0.2017 & 1.4 & 0.64 \\
\hline $02-189-3.1 \mathrm{c}$ & 0 & 0.079 & 0 & 246 & 0.33 & 1195.8 & 21.1 & 1172 & 41 & -2 & 2.22 & 2.8 & 0.2036 & 1.8 & 0.66 \\
\hline $02-189-3.2 \mathrm{c}$ & 0.000024 & 0.0785 & 0.04 & 340 & 0.57 & 1198.6 & 7.8 & 1151 & 11 & -4 & 2.2 & 0.9 & 0.2039 & 0.7 & 0.775 \\
\hline $02-189-4.1 \mathrm{c}$ & 0.000007 & 0.0784 & 0.01 & 547 & 0.32 & 1168.4 & 9 & 1154 & 16 & -1 & 2.14 & 1.2 & 0.1986 & 0.8 & 0.693 \\
\hline $02-189-4.2 \mathrm{r}$ & 0.000156 & 0.0787 & 0.26 & 802 & 0.24 & 1206.8 & 9.6 & 1108 & 31 & -9 & 2.16 & 1.7 & 0.2049 & 0.8 & 0.466 \\
\hline $02-189-5.1 \mathrm{c}$ & 0.000113 & 0.0782 & 0.19 & 182 & 0.31 & 1081.7 & 8 & 1111 & 25 & 3 & 1.93 & 1.5 & 0.1829 & 0.8 & 0.52 \\
\hline $02-189-5.2 \mathrm{c}$ & 0.00004 & 0.0811 & 0.07 & 215 & 0.32 & 1133.9 & 8.1 & 1209 & 27 & 6 & 2.14 & 1.6 & 0.193 & 0.7 & 0.465 \\
\hline $02-189-5.3 r$ & 0.000013 & 0.0748 & 0.02 & 497 & 0.17 & 951 & 9.6 & 1059 & 18 & 10 & 1.64 & 1.4 & 0.1597 & 1 & 0.766 \\
\hline $02-189-6.1 \mathrm{c}$ & 0.000028 & 0.0804 & 0.05 & 435 & 0.39 & 1152.5 & 7.5 & 1196 & 10 & 4 & 2.16 & 0.8 & 0.1962 & 0.7 & 0.794 \\
\hline $02-189-7.1 \mathrm{c}$ & 0.000016 & 0.0806 & 0.03 & 747 & 0.31 & 1141.3 & 7.9 & 1207 & 19 & 5 & 2.15 & 1.2 & 0.1943 & 0.7 & 0.596 \\
\hline $02-189-8.1 \mathrm{c}$ & -0.000007 & 0.0778 & -0.01 & 548 & 0.14 & 1137.2 & 12.1 & 1143 & 43 & 1 & 2.07 & 2.4 & 0.193 & 1.1 & 0.444 \\
\hline $02-189-8.2 \mathrm{r}$ & 0.0001 & 0.0772 & 0.17 & 676 & 0.14 & 1095.4 & 7 & 1089 & 36 & -1 & 1.93 & 1.9 & 0.1852 & 0.6 & 0.337 \\
\hline $02-189-9.1 \mathrm{c}$ & 0.000037 & 0.0802 & 0.06 & 322 & 0.31 & 1143.9 & 7.9 & 1189 & 32 & 4 & 2.14 & 1.8 & 0.1946 & 0.7 & 0.393 \\
\hline $02-189-10.1 \mathrm{r}$ & 0.000064 & 0.0738 & 0.11 & 168 & 0.22 & 1026.5 & 7.8 & 1012 & 24 & -1 & 1.73 & 1.4 & 0.1725 & 0.8 & 0.553 \\
\hline $02-189-11.1 \mathrm{c}$ & 0.000014 & 0.0801 & 0.02 & 296 & 0.31 & 1082.2 & 7.3 & 1194 & 17 & 9 & 2.02 & 1.1 & 0.1837 & 0.7 & 0.636 \\
\hline $02-189-12.1 \mathrm{c}$ & -0.000021 & 0.0791 & -0.04 & 389 & 0.31 & 1108.5 & 12.6 & 1182 & 33 & 6 & 2.06 & 2.1 & 0.1883 & 1.2 & 0.572 \\
\hline $02-189-13.1 \mathrm{c}$ & 0.000014 & 0.0806 & 0.02 & 495 & 0.31 & 1146.9 & 16 & 1207 & 26 & 5 & 2.17 & 2 & 0.1953 & 1.4 & 0.735 \\
\hline $02-189-13.2 \mathrm{r}$ & 0.000023 & 0.0777 & 0.04 & 482 & 0.31 & 1183.1 & 12.1 & 1132 & 29 & -5 & 2.15 & 1.8 & 0.201 & 1.1 & 0.583 \\
\hline $02-189-14.1 \mathrm{c}$ & 0 & 0.0785 & 0 & 343 & 0.36 & 1124.9 & 10.8 & 1159 & 24 & 3 & 2.07 & 1.5 & 0.1909 & 1 & 0.642 \\
\hline $02-189-15.1 \mathrm{c}$ & 0.000038 & 0.0809 & 0.06 & 276 & 0.38 & 1143 & 8.1 & 1205 & 27 & 5 & 2.15 & 1.5 & 0.1946 & 0.7 & 0.468 \\
\hline $02-189-16.1 \mathrm{r}$ & 0.000012 & 0.0736 & 0.02 & 1309 & 0.07 & 1002.2 & 6 & 1027 & 19 & 2 & 1.71 & 1.1 & 0.1684 & 0.6 & 0.538 \\
\hline $02-189-16.2 \mathrm{c}$ & 0.000009 & 0.0877 & 0.01 & 526 & 1.26 & 1333.4 & 8.3 & 1372 & 8 & 3 & 2.78 & 0.8 & 0.2303 & 0.6 & 0.846 \\
\hline $02-189-16.3 \mathrm{c}$ & 0 & 0.0788 & 0 & 224 & 0.35 & 1138.1 & 13.4 & 1167 & 25 & 2 & 2.1 & 1.7 & 0.1933 & 1.2 & 0.695 \\
\hline $02-189-17.1 \mathrm{r}$ & 0.00001 & 0.0743 & 0.02 & 503 & 0.62 & 1003.7 & 6.6 & 1045 & 12 & 4 & 1.72 & 0.9 & 0.1688 & 0.7 & 0.76 \\
\hline $02-189-17.2 \mathrm{c}$ & 0.000044 & 0.0792 & 0.07 & 223 & 0.41 & 1116.7 & 11 & 1162 & 24 & 4 & 2.05 & 1.6 & 0.1895 & 1 & 0.646 \\
\hline $02-189-17.3 \mathrm{r}$ & 0.003651 & 0.1208 & 6.31 & 83 & 1.22 & 989.5 & 9.3 & 881 & 184 & -12 & 1.56 & 9 & 0.1652 & 1.2 & 0.132 \\
\hline
\end{tabular}

a Sample names containing the suffix " $\mathrm{m}$ " are monazite; all other samples are zircon.

b ${ }^{206} \mathrm{~Pb} /{ }^{238} \mathrm{U}$ ages corrected for common $\mathrm{Pb}$ using the ${ }^{207} \mathrm{~Pb}$-correction method; ${ }^{207} \mathrm{~Pb} /{ }^{206} \mathrm{~Pb}$ ages corrected for common $\mathrm{Pb}$ using the ${ }^{204} \mathrm{~Pb}$-correction method. Decay constants from $\mathrm{Steiger}$ and Jäger (1977).

c 1-Sigma errors.

${ }^{d}$ Radiogenic ratios, corrected for common $\mathrm{Pb}$ using the ${ }^{204} \mathrm{~Pb}$-correction method, based on the Stacey and Kramers (1975) model.

All coordinates refer to UTM Grid Zone 17 NAD 27. 
Table 3

Summary of U-Pb SHRIMP geochronologic data for the Virginia Blue Ridge province in northern Virginia

\begin{tabular}{|c|c|c|c|c|c|c|c|}
\hline $\begin{array}{l}\text { Magmatic } \\
\text { Interval }\end{array}$ & $\begin{array}{l}\text { Lithologic } \\
\text { unit }\end{array}$ & Sample & $\begin{array}{l}\text { Sample location } \\
\text { (Fig. 3) }\end{array}$ & Figure & $\begin{array}{l}\text { Igneous } \\
\text { crystallization } \\
\text { age (Ma) }\end{array}$ & $\begin{array}{l}\text { Metamorphic } \\
\text { recrystallization } \\
\text { age }(\mathrm{Ma})\end{array}$ & $\begin{array}{l}\text { Inheritance } \\
\text { (Ma) }\end{array}$ \\
\hline \multirow[t]{6}{*}{ III } & Ybg & SNP-02-186 & 15 & Fig. 5B & $1028 \pm 9$ & & $\sim 1.2 \mathrm{Ga}$ \\
\hline & Yfqj $^{\mathrm{a}}$ & SNP-99-93 & 14 & - & $1050 \pm 8$ & $1018 \pm 14$ & \\
\hline & Ygr $^{\mathrm{a}}$ & OR-97-35 & 13 & - & $\begin{array}{l}1060 \pm 5 \\
1059 \pm 8(\mathrm{~m})\end{array}$ & $\begin{array}{l}1027 \pm 9\left(\mathrm{~m}^{\mathrm{b}}\right) \\
1019 \pm 15 \\
979 \pm 11\end{array}$ & $\sim 1.2-1.3 \mathrm{Ga}$ \\
\hline & Ygg & SNP-02-197 & 12 & Fig. 5D & $\begin{array}{l}1063 \pm 8 \\
1059 \pm 6(\mathrm{~m})\end{array}$ & $1010 \pm 14(\mathrm{~m})$ & $\sim 1090-1160$ \\
\hline & Ygg & SNP-01-170 & 11 & Fig. 5F & Undetermined & $0.94-0.99 \mathrm{Ga}$ & $\sim 1.2 \mathrm{Ga}$ \\
\hline & Ylgg ${ }^{a}$ & SNP-99-90 & 10 & - & $1078 \pm 9$ & $\begin{array}{l}1028 \pm 10 \\
997 \pm 19\end{array}$ & \\
\hline \multirow[t]{5}{*}{ II } & Yfe & SNP-03-204 & 9 & Fig. 6B & $1111 \pm 16$ & $1051 \pm 20$ & $\sim 1.2-1.3 \mathrm{Ga}$ \\
\hline & Yfpg & SNP-02-177 & 8 & Fig. 6D & $1120 \pm 12$ & $1050 \pm 12$ & $1178 \pm 14$ \\
\hline & Yfh & SNP-01-146 & 7 & Fig. 6F & $1144 \pm 8$ & - & $\sim 1.2 \mathrm{Ga}$ \\
\hline & $\mathrm{Ycf}^{\mathrm{a}}$ & SNP-96-10 & 6 & - & $1159 \pm 14$ & $1052 \pm 14$ & $\sim 1.2 \mathrm{Ga}$ \\
\hline & Ypqd & SNP-02-175 & 5 & Fig. 7B & $1161 \pm 10$ & $\begin{array}{l}1095 \pm 22 \\
0.96-1.04 \mathrm{Ga}\end{array}$ & $1.2-1.3 \mathrm{Ga}$ \\
\hline \multirow[t]{5}{*}{ I } & $\mathrm{Yg}$ & SNP-96-1 & 4 & Fig. 7D & $1164 \pm 8$ & $1.03-1.06 \mathrm{Ga}$ & $\sim 1.2-1.3 \mathrm{Ga}$ \\
\hline & $\mathrm{Yg}$ & SNP-03-198 & 3 & Fig. 7F & Undetermined & $0.98-1.04 \mathrm{Ga}$ & $\sim 1.2 \mathrm{Ga}$ \\
\hline & Ylgn & SNP-01-150 & 2 & Fig. 8B & $1165 \pm 7$ & ca. 1200 & $\sim 1.2 \mathrm{Ga}$ \\
\hline & Ylg2 & SNP-03-199 & 1 & Fig. 8D & $1175 \pm 11$ & $\begin{array}{l}1131 \pm 7 \\
1045 \pm 11\end{array}$ & $\sim 1.2-1.3 \mathrm{Ga}$ \\
\hline & Ylg1 & SNP-02-189 & 1 & Fig. $8 \mathrm{~F}$ & $1183 \pm 11$ & $1.01-1.09 \mathrm{Ga}$ & $\sim 1.2-1.3 \mathrm{Ga}$ \\
\hline
\end{tabular}

${ }^{\text {a }}$ Data from Tollo et al. (2004a).

b Monazite; all other ages from zircon.

\subsection{Results from $U-P b$ geochronology}

\subsubsection{Biotite-rich monzogranite to quartz monzodiorite, sample SNP-02-186}

Biotite-rich monzogranite to quartz monzodiorite (Ybg) was collected for isotopic analysis in the Madison quadrangle (locality 15, Fig. 3). The outcrop is composed of foliated, medium- to coarse-grained, biotite-rich granitoid that contains several meter-scale xenoliths of leucocratic granite from the neighboring Ylg complex (Bailey et al., 2003; Tollo et al., 2004c). Zircons from this rock are light brown to colorless, elongate (length-to-width ratio $(l / w)$ of 3-5), and typically clear with few inclusions or imperfections. Most grains have unusual CL zoning that is indistinct and irregular; a few zircons show faint oscillatory zoning (Fig. 5A). This pale CL zoning is reflective of the very low uranium concentrations (about 17-75 ppm) of the zircons which result in relatively imprecise ${ }^{207} \mathrm{~Pb} /{ }^{206} \mathrm{~Pb}$ ages (Table 2). SHRIMP results indicate that 16 of 20 analyses (14 cores and 2 overgrowths) are concordant and yield a Concordia Age of $1028 \pm 9 \mathrm{Ma}$ (Fig. 5B) that we interpret as the time of igneous crystallization. Three analyses of overgrowths are slightly younger (ages of about $1.0 \mathrm{Ga}$ ) and two analyses of cores are older (ages $\sim 1.25 \mathrm{Ga}$ ).

\subsubsection{Garnetiferous syenogranite, samples SNP-02-197 and SNP-01-170}

Two samples of garnetiferous syenogranite (Ygg) were collected in the Chester Gap quadrangle (localites 12 and 11, Fig. 3). The outcrop at locality 12 is composed of weakly foliated, medium-grained, garnet + biotite leucogranite. The outcrop at locality 11 is composed of weakly foliated, medium- to coarse-grained garnet + biotite syenogranite that is typical of the main pluton.

Zircons from both samples occur as two distinct morphologies (Fig. 5C and E). Elongate $(l / w=3-4)$ grains are medium to dark brown and typically contain numerous fractures that are mostly concentrated in core regions. CL imaging shows that most of these grains are composed of partially resorbed cores of igneous ori- 
(A)

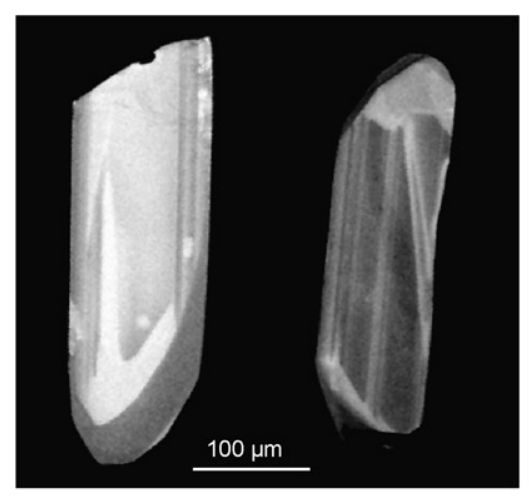

(C)

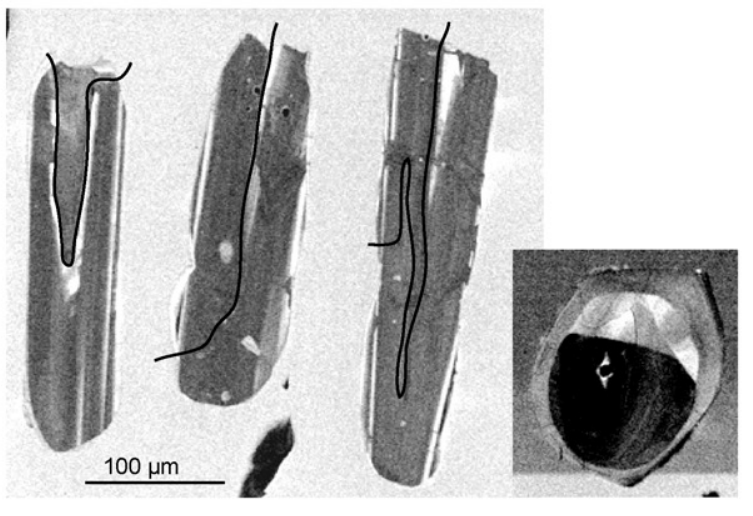

(E)

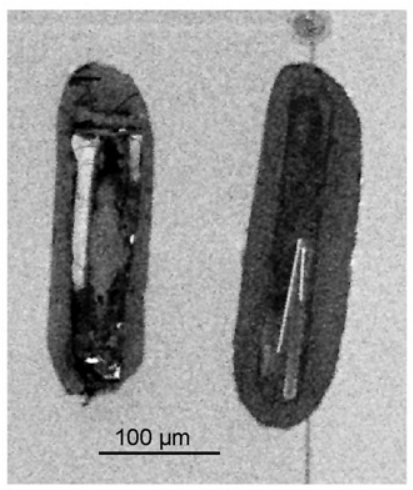

(B)

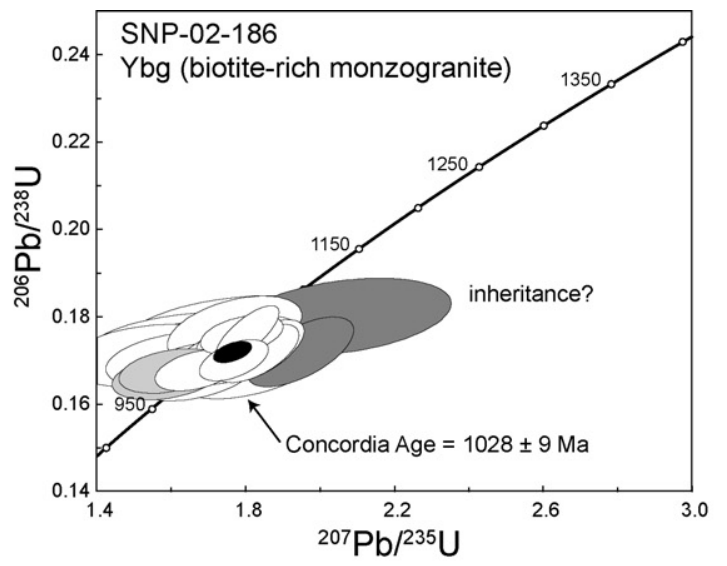

(D)

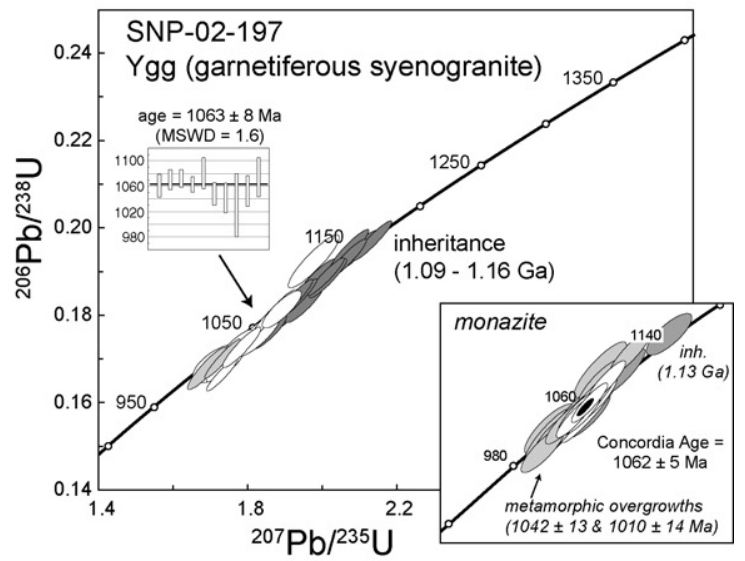

(F)

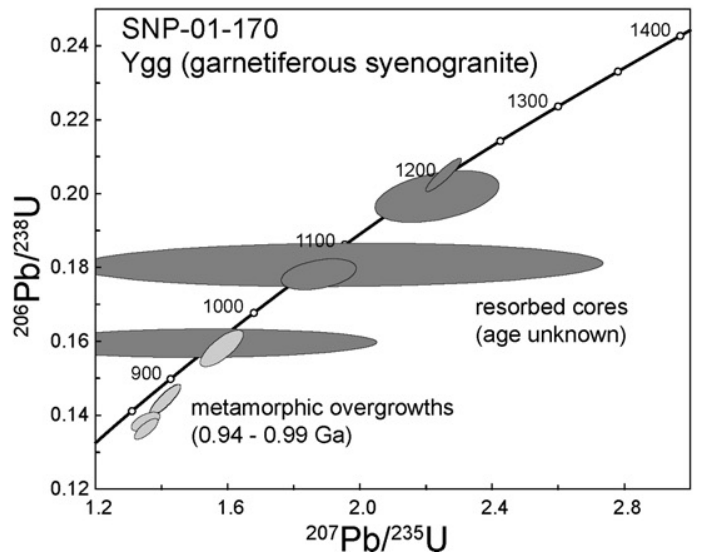

Fig. 5. CL images of zircons (left) and Concordia and weighted averages plots of SHRIMP isotopic data (right) obtained by analyses of zircons from biotite-rich monzogranite (Ybg, A and B) and garnetiferous syenogranite (Ygg, sample SNP-02-197, C and D; sample SNP-01-170, E and F). Solid lines in $\mathrm{C}$ separate observed cores and overgrowths.

gin with remnant oscillatory zoning and large, dark (in $\mathrm{CL}$ ), unzoned metamorphic overgrowths. The darkness of the CL images is indicative of the high $\mathrm{U}$ content (mostly 1000-2000 ppm) of zircons from both samples. Core material has $\mathrm{Th} / \mathrm{U}$ of $0.16-0.41$, typical of igneous zircon, whereas overgrowths have lower Th/U (0.01-0.05) typical of metamorphic origin. Metamorphic material occurs both around and within the damaged older cores (Fig. 5C and E). The proportion of igneous cores generally ranges from about 10 to $50 \%$; some 
grains contain no apparent core material. Because the cores are small, resorbed, fractured, and locally invaded by metamorphic zircon, it was difficult to locate pristine areas for SHRIMP analysis. Also present are equant grains $(l / w=1)$, each of which is composed of a fragment of an oscillatory-zoned zircon overgrown by a thick metamorphic rim (Fig. 5C).

Monazite from SNP-02-197 is pale yellow and anhedral to subhedral. In BSE, most grains show patchyor oscillatory-zoned cores and broad, unzoned rims. The zoning distinctions are also reflected in chemical data. Although cores and rims have similar ranges of Th concentrations ( $\sim 6000-10,000 \mathrm{ppm})$, concentrations of $U$ in cores $(\sim 500-1100 \mathrm{ppm})$ is higher than in rims ( $\sim 400-600 \mathrm{ppm})$. Th/U in cores $(\sim 6-16)$ is somewhat lower than in rims $(\sim 13-28)$.

Ten analyses of zircon from sample SNP-02-197 yield a coherent age of $1063 \pm 8 \mathrm{Ma}$ (Fig. 5D). Some zircons contain inherited cores with ages ranging from 1.09 to $1.16 \mathrm{Ga}$. Primary, oscillatory-zoned regions are crosscut by relatively unzoned material (analogous to overgrowths) two analyses of which yielded ages of about 1.01 and $1.03 \mathrm{Ga}$. The second sample (SNP-01170) produced very poorly preserved zircons from which it was impossible to obtain a magmatic age due to the very small size of the remnant igneous cores (Fig. 5E). Overgrowths have typically low $\mathrm{Th} / \mathrm{U}(0.06-0.07)$ and yielded metamorphic ages of about $0.94-0.99 \mathrm{Ga}$ (Fig. 5F).

Isotopic data from 11 cores of monazite from sample SNP-02-197 result in a Concordia Age of $1062 \pm 5 \mathrm{Ma}$, in agreement with data from cores of co-existing zircon (Fig. 5D). Somewhat older ages ( $1090-1130 \mathrm{Ma})$ were obtained from three oscillatory-zoned cores, indicating the occurrence of inherited material in some of the monazites. Two ages $(1046 \pm 13$ and $1010 \pm 14 \mathrm{Ma}$, based on five analyses each) of rims are in agreement with very limited data from rims of co-existing zircon.

\subsubsection{Equigranular pyroxene monzogranite (farsundite), sample SNP-03-204}

Weakly foliated, medium-grained, equigranular, opxbearing monzogranite (Yfe) was collected in the Big Meadows quadrangle (locality 9, Fig. 3). Zircons are medium brown and elongate $(l / w=3-4)$. CL imaging shows that the cores are broadly oscillatory-zoned (Fig. 6A); a few grains contain very small, dark overgrowths. Uranium content of oscillatory-zoned cores is low (mostly 100-200 ppm) and yielded relatively imprecise ${ }^{207} \mathrm{~Pb} /{ }^{206} \mathrm{~Pb}$ ages (Table 2). Sixteen analyses overlap in an array between about 1.0 and $1.2 \mathrm{Ga}$ (Fig. 6B). The weighted average of these analyses is $1098 \pm 18 \mathrm{Ma}$
(MSWD $=3.1$ ). However, subdividing this group solely on the basis of age (most grains have similar morphologies) results in ages of $1111 \pm 16 \mathrm{Ma}(\mathrm{MSWD}=1.7$; 12 analyses) and $1051 \pm 20$ (MSWD $=0.24 ; 4$ analyses). Further complicating the interpretation is that each subgroup contains data from an apparent overgrowth. We cautiously interpret a crystallization age of $1111 \pm 16 \mathrm{Ma}$. Inherited material is also present, ranging in age from about 1.19 to $1.34 \mathrm{Ga}$.

\subsubsection{Foliated pyroxene monzogranite (farsundite), sample SNP-02-177}

Strongly foliated, medium- to coarse-grained, pyroxene monzogranite (Yfpg) was collected in the Chester Gap quadrangle (locality 8, Fig. 3). Zircons from this sample are dark brown and stubby to elongate $(l / w=2-4)$. Many grains contain partially resorbed cores (presumably inherited), broad igneous mantles showing fine oscillatory zoning in CL, and moderate-sized, dark (in CL), unzoned overgrowths (Fig. 6C). Uranium concentrations of both mantles and overgrowths is intermediate (mostly 200-700 ppm) (Table 2). Fourteen analyses of the oscillatory-zoned mantles yield an age of $1120 \pm 12 \mathrm{Ma}$, interpreted as the time of crystallization (Fig. 6D). An older (1178 $\pm 14 \mathrm{Ma})$ generation of oscillatory-zoned zircon is locally similar in appearance to the 1120 Ma mantles. However, a few resorbed cores are also this age, suggesting that this $1178 \mathrm{Ma}$ material was inherited from the source. Overgrowths are dated at $1050 \pm 12 \mathrm{Ma}$.

\subsubsection{Flint Hill Gneiss, sample SNP-01-146}

Strongly foliated, medium-grained, syenogranite gneiss (Yfh) that locally displays centimeter- to decimeter-scale compositional layering oriented parallel to foliation was collected in the Chester Gap quadrangle (locality 7, Fig. 3). Zircons are light to medium brown and stubby to somewhat elongate $(l / w=2-3)$. Most grains are composed of igneous mantles of finely oscillatory-zoned material overgrown by thin, dark (in CL) rims of metamorphic origin; some grains contain inherited cores (Fig. 6E). Uranium concentrations are intermediate (mostly 300-600 ppm) (Table 2). Sixteen analyses (13 cores and 3 overgrowths) yield an age of $1144 \pm 8 \mathrm{Ma}$, interpreted as the time of protolith crystallization (Fig. 6F). Inherited material of circa $1.2 \mathrm{Ga}$ age also is present in some grains. Four young ages (about 1.03-1.10 Ga) are from oscillatoryzoned grains that are similar in appearance to the older grains. These analyses are excluded from the age calculation because of their significantly younger age. 
(A)

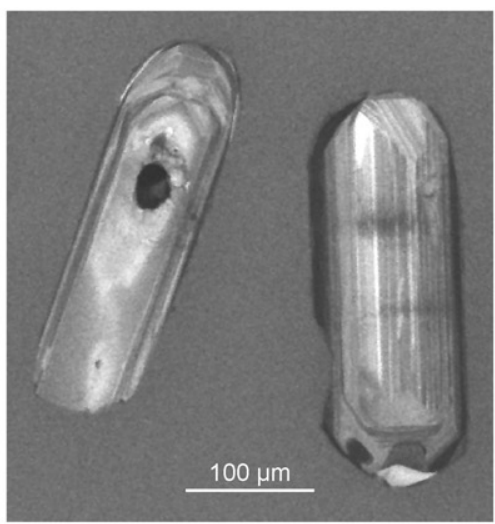

(C)

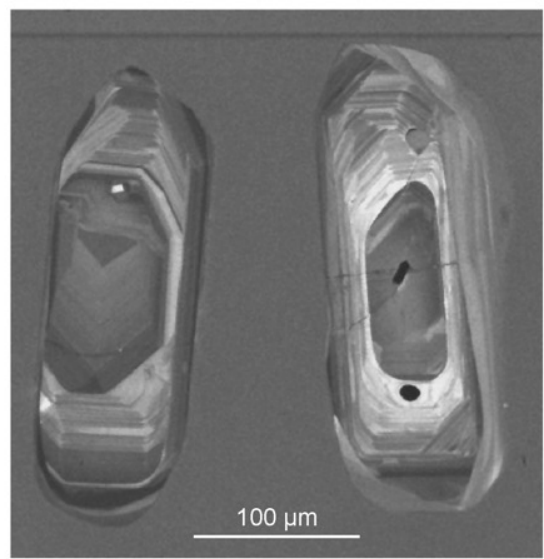

(E)

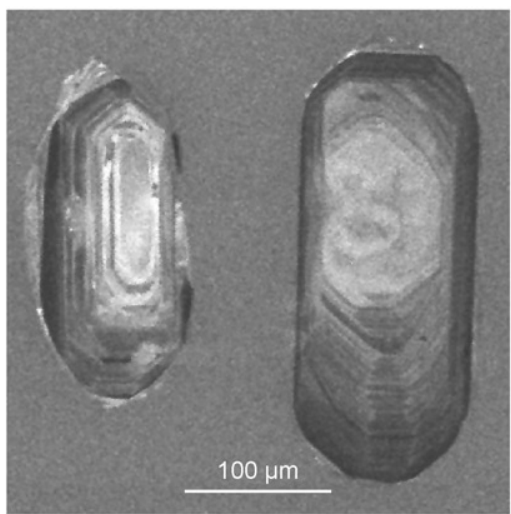

(B)

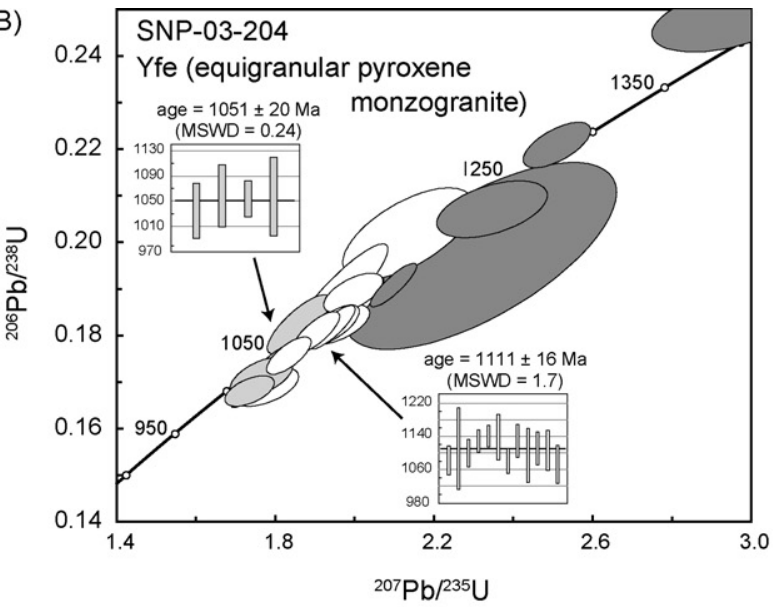

(D)

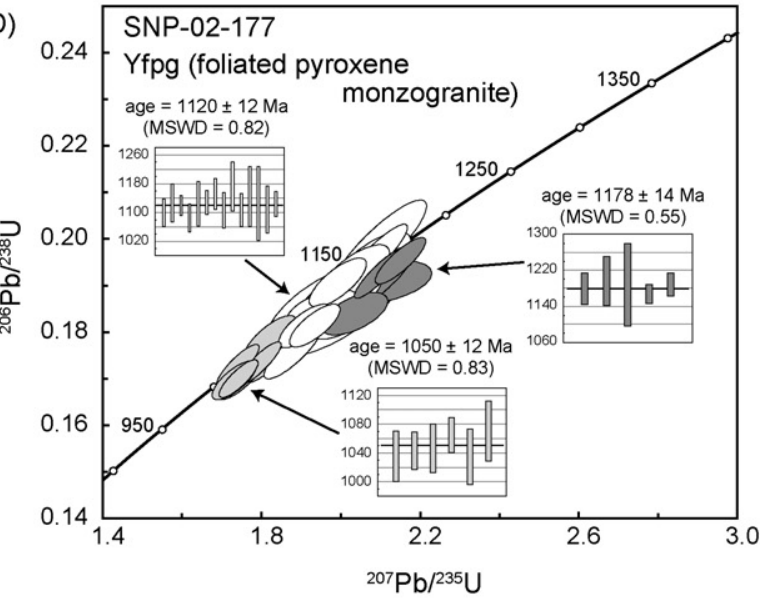

( $\mathrm{F}$

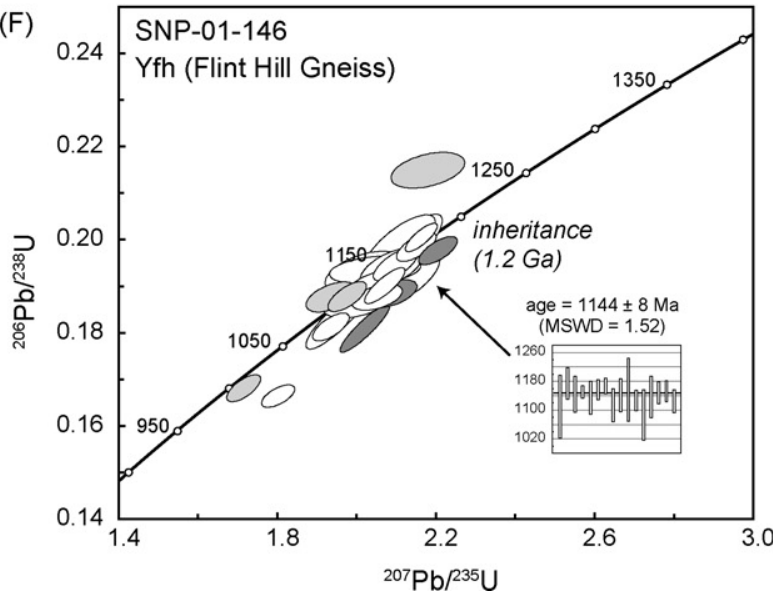

Fig. 6. CL images of zircons (left) and Concordia and weighted averages plots of SHRIMP isotopic data (right) obtained by analyses of zircons from equigranular pyroxene monzogranite (Yfe, A and B), foliated pyroxene monzogranite (Yfpg, C and D), and Flint Hill Gneiss (Yfh, E and F). 


\subsubsection{Pyroxene quartz diorite (orthopyroxene quartz diorite), sample SNP-02-175}

Strongly foliated, medium-grained, opx + biotitebearing quartz diorite (Ypqd) was collected in the Chester Gap quadrangle (locality 5, Fig. 3). The roadcut exhibits weak compositional layering that is locally deformed into tight folds with axial planes oriented parallel to foliation. Zircons are light to medium brown and either elongate $(l / w=3-4)$ or equant $(l / w=1)$. Igneous cores exhibit broad oscillatory zoning; overgrowths appear either very light or medium dark in CL (Fig. 7A). Cores contain relatively low U contents (mostly 100-200 ppm); most overgrowths are even lower in $\mathrm{U}$ (mostly $25-100 \mathrm{ppm}$ ). Both cores and overgrowths have similar $\mathrm{Th} / \mathrm{U}$ values (0.39-0.84).

Age data from this unit define multiple populations. Nine analyses result in a Concordia Age of $1161 \pm 10$ $\mathrm{Ma}$, interpreted as the time of protolith crystallization (Fig. 7B). Four broadly zoned zircons of generally similar appearance yield an apparent age of $1095 \pm 22 \mathrm{Ma}$; however, this age may be due to Pb-loss, not new growth of zircon. Three other grains yield older ages of about $1.23-1.30 \mathrm{Ga}$, and are considered to be inherited. Also present are distinctly younger grains with ages about $0.96-1.04 \mathrm{Ga}$ that are interpreted to have formed during regional metamorphism.

\subsubsection{Garnetiferous granite (charnockite to farsundite) gneiss, samples SNP-96-1 and SNP-03-198}

Two samples of strongly foliated, opx + garnetbearing syeno- to monzogranite (charnockite to farsundite) gneiss ( $\mathrm{Yg}$ ) were collected within an elongate inlier in the Thornton Gap quadrangle (localities 4 and 3, Fig. 3). Zircons from locality 4 are medium to dark brown and elongate $(l / w=2-4)$ (Fig. 7C). Most grains include oscillatory-zoned igneous mantles and wide, dark metamorphic overgrowths. Zircons from locality 3 are similar in shape and color (Fig. 7E). However, the proportion of overgrowth is much greater; in many grains, only a tiny remnant core remains, overgrown by extensive dark (in CL) rims. Uranium content of cores and overgrowths is intermediate (mostly about 150-600) (Table 2); both have relatively high $\mathrm{Th} / \mathrm{U}$ values (mostly $0.2-0.7$ ). Meaningful isotopic data indicative of the igneous age of the gneiss were obtained only from zircons from locality 4.

Twelve analyses from sample SNP-96-1 result in a Concordia Age of $1164 \pm 8 \mathrm{Ma}$ (Fig. 7D), interpreted as the time of crystallization of the protolith. Several other analyses have similar ${ }^{207} \mathrm{~Pb} /{ }^{206} \mathrm{~Pb}$ ages but are slightly discordant; these data were excluded from the
Concordia Age calculation. Ages of 1.22 and $1.32 \mathrm{Ga}$ from two oscillatory-zoned grains suggest that these zircons are xenocrysts. Also present are some dark (in CL) metamorphic overgrowths that yielded ages of 1.01-1.04 Ga. In zircons from sample SNP-03-198, $\mathrm{Th} / \mathrm{U}$ varies widely $(0.09-1.8)$ in both cores and overgrowths. Small, resorbed cores in sample SNP-03-198 did not yield a coherent age population (Fig. 7F). Metamorphic overgrowths from this sample are about $0.98-1.04 \mathrm{Ga}$.

\subsubsection{Layered granodiorite (opdalite) gneiss, sample SNP-01-150}

Strongly foliated, medium- to locally coarse-grained, opx + amphibole-bearing granodiorite (opdalite) gneiss (Ylgn) was collected in the Chester Gap quadrangle (locality 2, Fig. 3). Zircons are light to medium brown and elongate $(l / w=3-4)$ (Fig. 8A). Most grains are composed of oscillatory-zoned igneous mantles and small, dark (in CL) metamorphic overgrowths. The light color of the zircons is due to the relatively low $\mathrm{U}$ content (mostly 100-300 ppm). Seventeen analyses yield an age of $1165 \pm 7 \mathrm{Ma}$, interpreted as the time of protolith crystallization (Fig. 8B). One overgrowth, with low $\mathrm{Th} / \mathrm{U}$ of 0.18 , was dated at about $1.07 \mathrm{Ga}$; an inherited core yielded an age of about $1.25 \mathrm{Ga}$.

\subsubsection{Leucocratic granitoid and foliated megacrystic leucocratic granite (Ylg2 and Ylg1), samples SNP-03-199 and SNP-02-189, respectively)}

Two samples were collected from the leucogranite complex (Ylg) in the Madison quadrangle (locality 1, Fig. 3). Sample SNP-03-199 was collected from medium-grained, equigranular biotite leucogranite (Ylg2) that occurs as dikes which crosscut associated coarser-grained granitoid. Sample SNP-02-189 was collected from foliated megacrystic biotite-bearing leucocratic granite (Ylg1) exposed nearby.

Zircons from the medium-grained leucogranite (Ylg2) are medium to dark brown and either elongate $(l / w=3-4)$ or equant $(l / w=1)$ (Fig. 8C). All elongate grains are composed of highly fractured mantles that show fine oscillatory zoning and thin to moderate, dark (in CL), unzoned overgrowths. Some grains contain small, inherited cores. Uranium contents of mantles are moderate (mostly about 500-900 ppm) (Table 2). Ten analyses of oscillatory-zoned mantles yield two apparently distinct age groupings: $1175 \pm 11$ and $1131 \pm 7 \mathrm{Ma}$ (Fig. 8D). These groups are distinguished on the basis of age only; all grains in both groups have similar morphology. Overgrowths, some of which have low Th/U of $0.04-0.06$, are dated at $1045 \pm 11 \mathrm{Ma}$. Two possible 
(A)

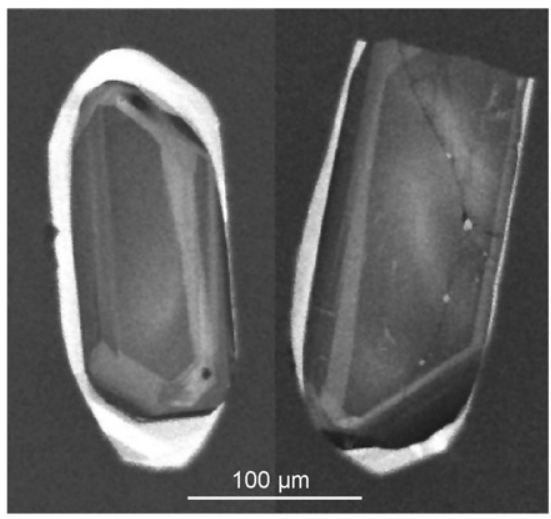

(C)

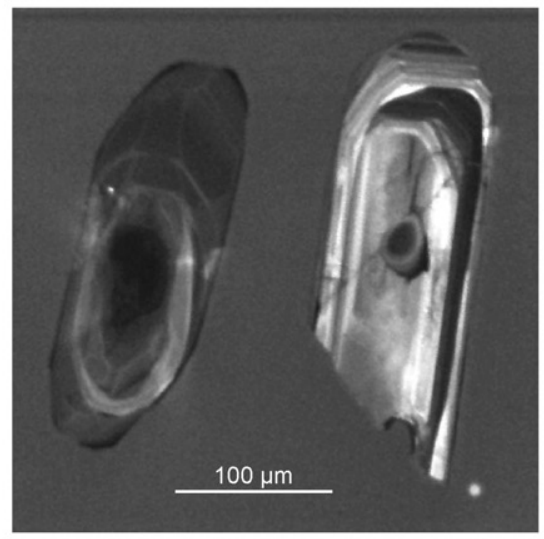

(E)

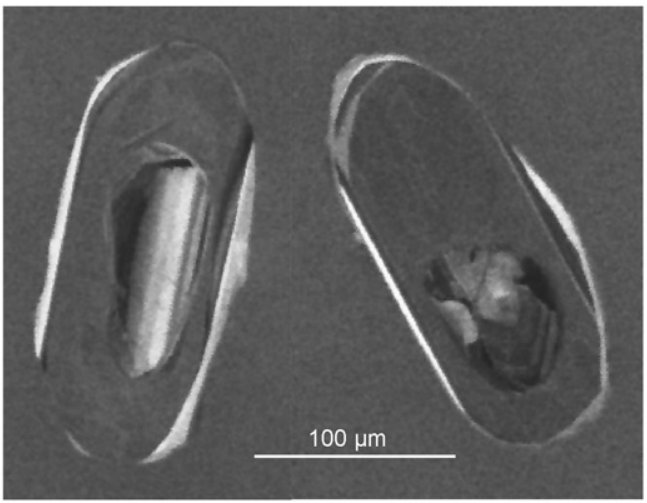

(B)

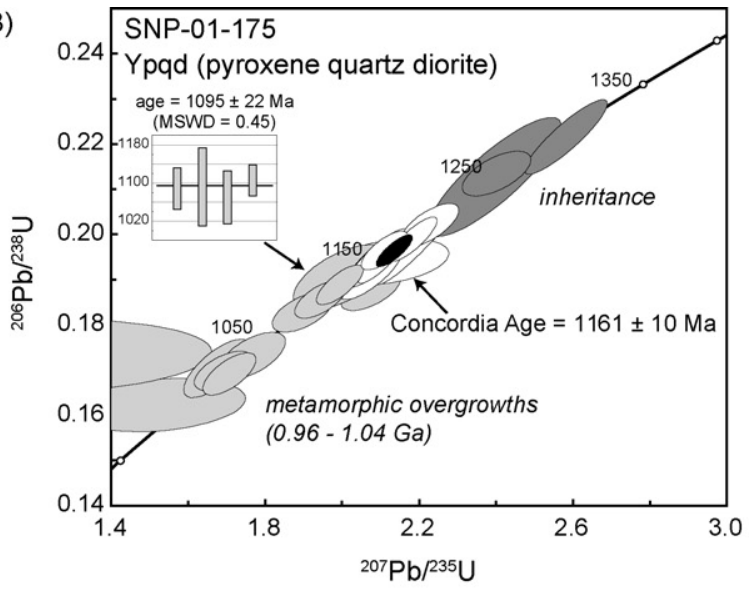

(D)

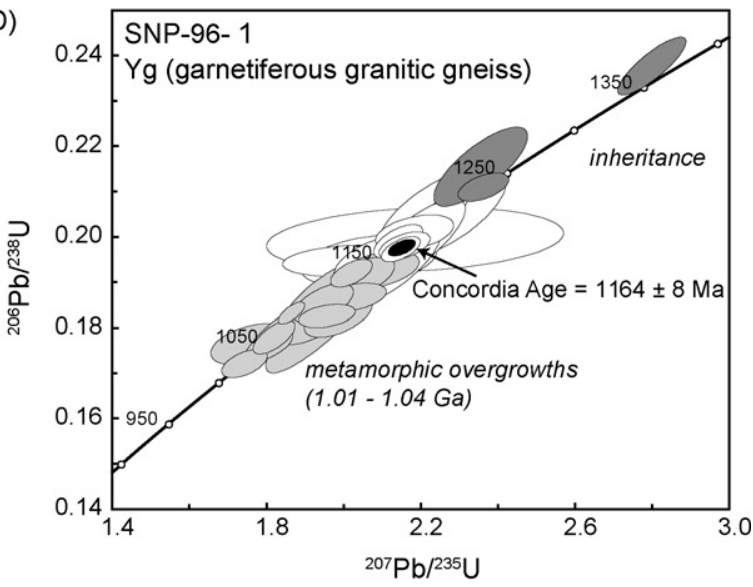

(F)

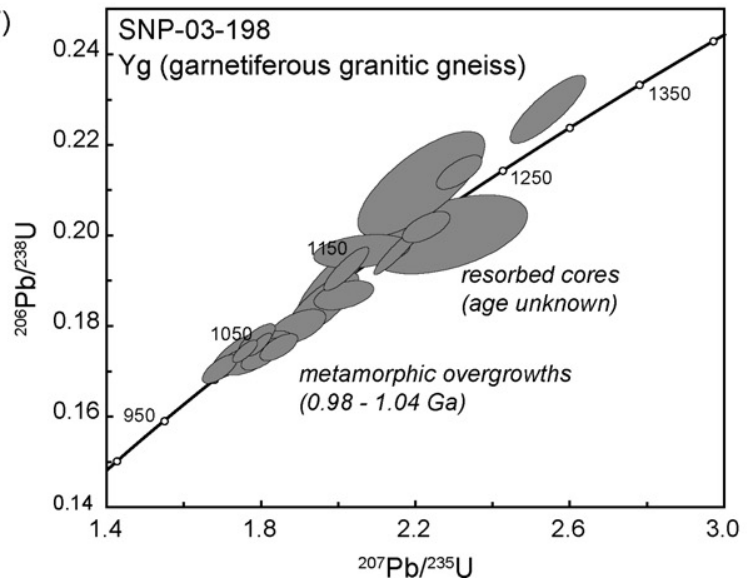

Fig. 7. CL images of zircons (left) and Concordia and weighted averages plots of SHRIMP isotopic data (right) obtained by analyses of zircons from orthopyroxene quartz diorite (Ypqd, A and B) and garnetiferous granite gneiss (Yg, sample SNP-96-1, C and D; sample SNP-03-198, E and F).

interpretations of the age data from the mantles are that the rock: (1) crystallized at about $1175 \mathrm{Ma}$ and was metamorphosed at about $1131 \mathrm{Ma}$, or (2) crystallized at about $1131 \mathrm{Ma}$ and contains xenocrystic $1175 \mathrm{Ma}$ zircons possibly derived from Ylg1.
Zircons from the megacrystic granite (Ylg1) are medium brown and elongate $(l / w=3-4)$ (Fig. $8 \mathrm{E})$. Most grains are composed of broad mantles showing fine oscillatory zoning and dark (in CL) overgrowths. Uranium contents (mostly about 200-500 ppm) of igneous 
(A)

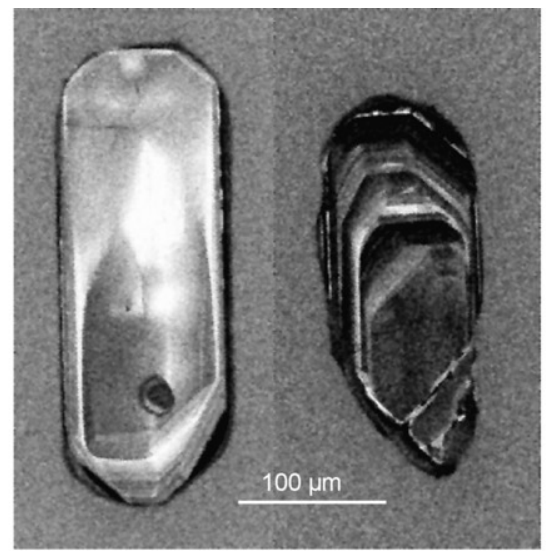

(C)

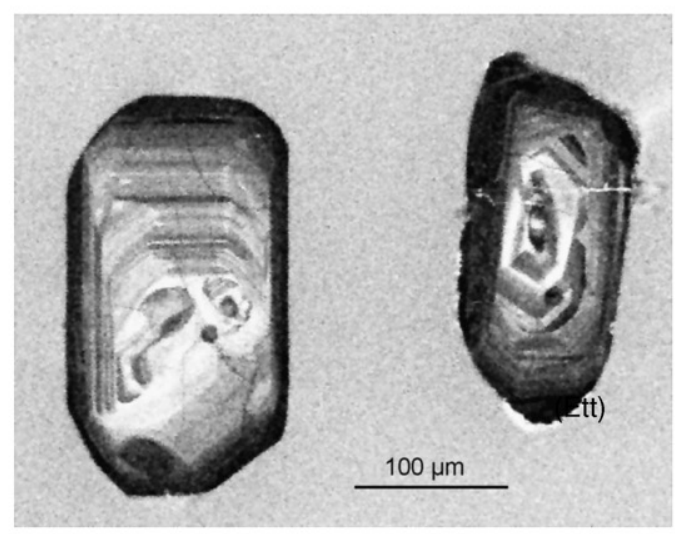

(E)

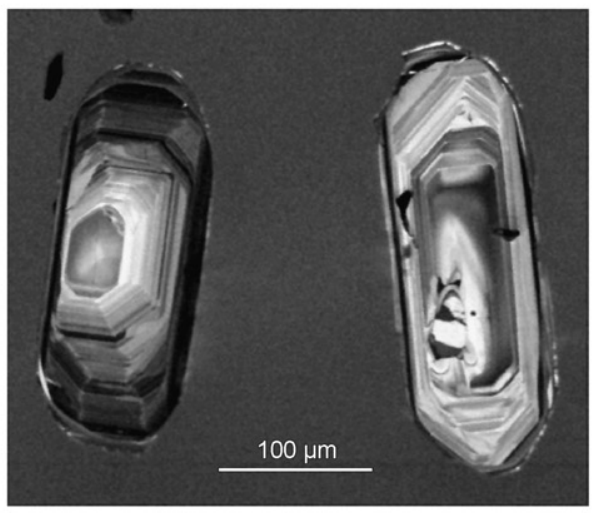

(B)

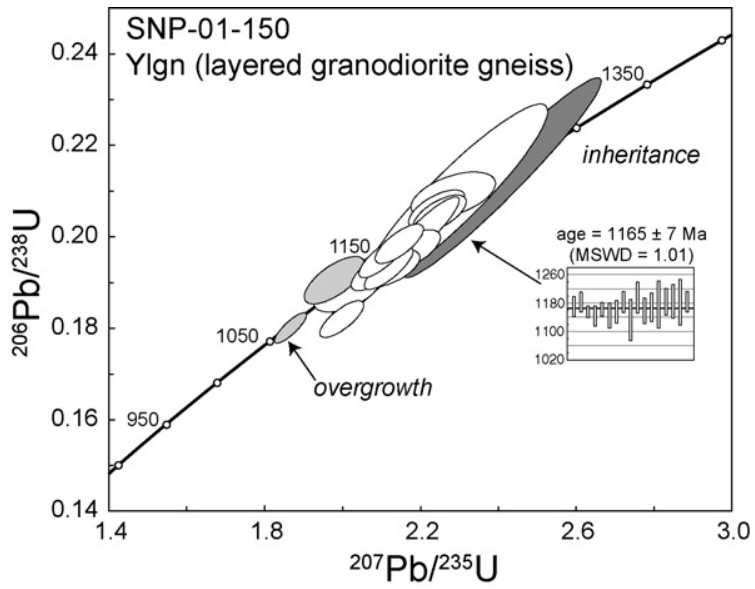

(D)

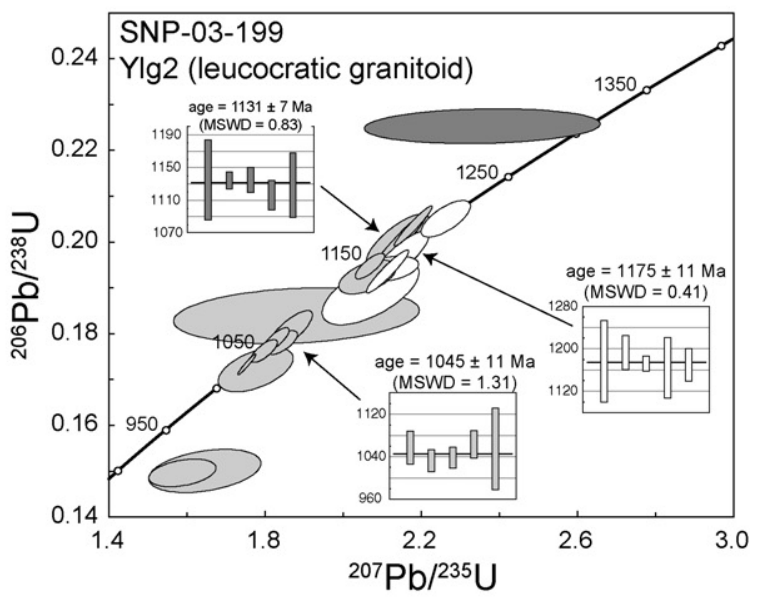

(F)

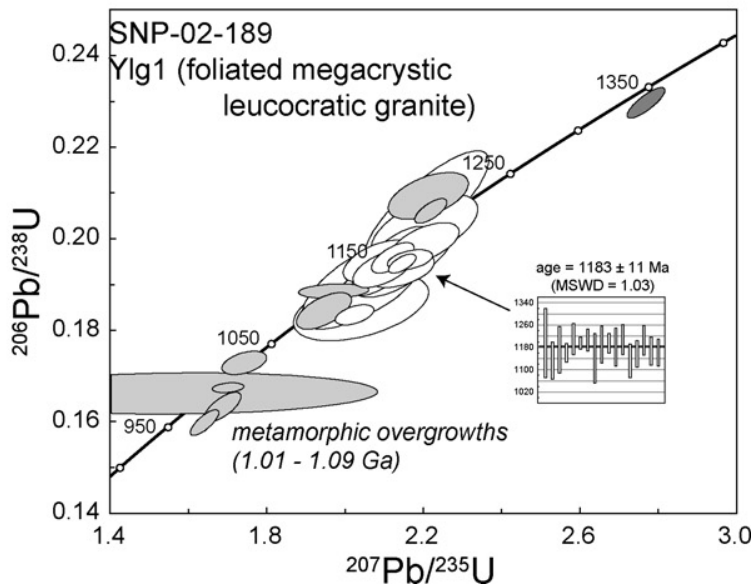

Fig. 8. CL images of zircons (left) and Concordia and weighted averages plots of SHRIMP isotopic data (right) obtained by analyses of zircons from layered granodiorite gneiss (Ylgn, A and B), leucocratic granitoid (Ylg2, C and D) and foliated megacrystic leucocratic granite (Ylg1, E and F).

mantles are lower than in zircons from Ylg2. Seventeen analyses yield a coherent age group at $1183 \pm 11 \mathrm{Ma}$ (Fig. 8F), interpreted as the igneous crystallization age. Five of six metamorphic overgrowths have relatively low
$\mathrm{Th} / \mathrm{U}$ values and have ages of about $1.01-1.09 \mathrm{Ga}$. The significantly different $\mathrm{U}$ contents of circa $1175-1185 \mathrm{Ma}$ zircons in Ylg2 and Ylg1 (Table 2) suggest that the older population of zircon in Ylg2 are not inherited from Ylg1. 
Thus, the combination of field relations, petrologic characteristics, and zircon systematics suggests that the leucocratic granitoid (Ylg2) formed at about $1175 \mathrm{Ma}$ (i.e. shortly after emplacement of Ylg1). Zircons of $1131 \mathrm{Ma}$ age in Ylg2 record a younger event, perhaps related to local generation of undated pegmatite.

\subsection{Summary of geochronologic data}

The new geochronologic data (Table 3), combined with ages reported by Aleinikoff et al. (2000) and Tollo et al. (2004a), indicate that Grenvillian magmatism in the Blue Ridge occurred during three major periods: Magmatic Interval I, 1183-1144 Ma; Magmatic Interval II, 1120-1111 Ma; and Magmatic Interval III, 1078-1028 Ma. A major episode of deformation peaked at $1078-1050 \mathrm{Ma}$ but continued to affect rocks for $20 \mathrm{~m}$.y. Isotopic evidence further suggests that temperatures remained elevated until about $980 \mathrm{Ma}$.

\section{Major- and trace-element geochemistry}

A total of 135 samples were analyzed for majorand trace-element compositions. Data for representative samples from each lithologic unit shown in Fig. 3 are presented in Table 4.

\subsection{Analytical methods}

Powders were prepared from samples weighing $20-40 \mathrm{~kg}$ by crushing using a steel jaw aparatus and grinding in a shatterbox. Powder subsplits were analyzed for major elements and trace elements including $\mathrm{Rb}, \mathrm{Ba}$, $\mathrm{Sr}, \mathrm{Pb}, \mathrm{Zr}, \mathrm{Nb}, \mathrm{Ni}, \mathrm{Zn}, \mathrm{Cr}, \mathrm{Ga}, \mathrm{V}$, and $\mathrm{Y}$ by $\mathrm{X}$-ray fluorescence (XRF) techniques at the Gilmore Laboratory at the University of Massachusetts in Amherst using methods modified from Norrish and Chappell (1967) and Norrish and Hutton (1969). All samples were analyzed in duplicate. Abundances of rare-earth elements (REE) and Th, $\mathrm{U}, \mathrm{Hf}, \mathrm{Ta}$, and Sc were determined using instrumental neutron activation techniques at Oregon State University.

\subsection{Major-element characteristics}

Whole-rock compositions define a broad data array encompassing a range of $\sim 30 \%$ in $\mathrm{SiO}_{2}$ content (Fig. 9). Compositional variation defines steep gradients in $\mathrm{TiO}_{2}$ and $\mathrm{P}_{2} \mathrm{O}_{5}$ contents (Fig. 9A and $\mathrm{B}$, respectively) which, together with relatively low $\mathrm{CaO}$ contents at low silica concentrations (Fig. 9E), are features characteristic of igneous charnockites (Kilpatrick and Ellis, 1992). Most lithologic units are silica-rich with $\mathrm{SiO}_{2}$ contents $>65 \%$. Considered in terms of lithologic types, all leucogranites are silicic, including the most silica-rich rocks in the region (Fig. 9). Non-leucocratic granitoids are broadly similar to the leucogranites but with generally lower $\mathrm{SiO}_{2}$ contents (Fig. 9). The petrologically unrelated low-silica charnockites (Ypqd and Yfqj) are characterized by silica contents $<65 \%$ and by typically high concentrations of compatible major elements (Fig. 9). Biotite-rich monzogranite (Ybg) spans the compositional gap between high- and low-silica rocks (Fig. 9). The only other lithologic unit of broadly intermediate composition, layered opx-bearing granodiorite gneiss (Ylgn), shares many chemical characteristics with the non-leucocratic granitoids and is considered part of that group (Table 1).

Nearly all rocks are subalkaline (Fig. 9F), and over $90 \%$ of the analyses plot within the ferroan field of Frost et al. (2001) (Fig. 9G), indicating strong tholeiitic affinity. However, samples of the circa 1160 Ma lowsilica charnockite (Ypqd) plot within the magnesian field and thus display compositional similarity to arc-related Cordilleran granites. Most Blue Ridge granitoids are characterized by marked K enrichment (Fig. 9H) regardless of age, presence or absence of opx, and degree of alteration. Low-silica charnockite of Magmatic Interval I (Ypqd) is compositionally distinct in this regard as well, characterized by only moderate $\mathrm{K}$ enrichment.

Aluminum saturation indices (ASI) vary considerably with rock type (Fig. 10). All leucogranitic units are peraluminous (ASI > 1.0) (Fig. 10A and C), whereas non-leucocratic granitoids (with or without opx) are borderline metaluminous to weakly peraluminous (ASI $=0.95-1.1)$ (Fig. 10B and C). Low-silica charnockites are borderline to strongly metaluminous (ASI $\leq 0.7-1.1)$ (Fig. 10A and C) and the intermediate biotite monzogranite ( $\mathrm{Ybg}$ ) is transitional between the high- and low-silica groupings (Fig. 10A). Considered in terms of age, granitoids of Magmatic Interval III show the greatest compositional variation, defining a continuum of compositions ranging from strongly metaluminous to moderately peraluminous and spanning the entire silica spectrum of the regional suite (Fig. 10A). Granitoids emplaced during Magmatic Intervals II and I are borderline metaluminous (dominantly charnockites) to moderately peraluminous (leucogranites) (Fig. 10B and $\mathrm{C}$ ).

\subsection{Trace-element characteristics}

Mesoproterozoic rocks of the northern Blue Ridge display systematic variation in trace-element concen- 
Table 4

Geochemical compositions of representative samples

\begin{tabular}{|c|c|c|c|c|c|c|c|c|}
\hline $\begin{array}{l}\text { Map unit } \\
\text { sample }\end{array}$ & $\begin{array}{c}\text { Ylg1 (z) } \\
\text { SNP-02-189Z }\end{array}$ & $\begin{array}{l}\text { Ylg2 (z) } \\
\text { SNP-03-199 }\end{array}$ & $\begin{array}{c}\text { Ylgn } \\
\text { SNP-01-148 }\end{array}$ & $\begin{array}{l}\text { Ylgn (z) } \\
\text { SNP-01-150 }\end{array}$ & $\begin{array}{c}\text { Yg } \\
\text { SNP-96-5 }\end{array}$ & $\begin{array}{c}\text { Yg (z) } \\
\text { SNP-03-198 }\end{array}$ & $\begin{array}{c}\text { Ypqd } \\
\text { SNP-01-154 }\end{array}$ & $\begin{array}{l}\text { Ypqd (z) } \\
\text { SNP-01-175 }\end{array}$ \\
\hline $\mathrm{SiO}_{2}{ }^{\mathrm{a}}$ & 72.01 & 75.67 & 64.89 & 66.67 & 68.77 & 72.09 & 56.68 & 55.29 \\
\hline $\mathrm{TiO}_{2}$ & 0.39 & 0.14 & 0.94 & 0.80 & 0.61 & 0.41 & 1.45 & 1.71 \\
\hline $\mathrm{Al}_{2} \mathrm{O}_{3}$ & 14.28 & 12.65 & 15.86 & 15.53 & 14.67 & 14.28 & 17.81 & 16.49 \\
\hline $\mathrm{Fe}_{2} \mathrm{O}_{3}{ }^{\mathrm{b}}$ & 2.71 & 0.85 & 5.73 & 4.97 & 4.19 & 3.00 & 8.35 & 9.33 \\
\hline $\mathrm{MnO}$ & 0.04 & 0.02 & 0.07 & 0.08 & 0.06 & 0.04 & 0.09 & 0.14 \\
\hline $\mathrm{MgO}$ & 0.42 & 0.01 & 1.22 & 0.85 & 0.63 & 0.43 & 3.53 & 4.78 \\
\hline $\mathrm{CaO}$ & 1.58 & 0.61 & 4.13 & 3.49 & 2.33 & 1.76 & 5.40 & 6.54 \\
\hline $\mathrm{Na}_{2} \mathrm{O}$ & 2.63 & 3.02 & 3.13 & 2.96 & 2.94 & 2.53 & 3.50 & 3.00 \\
\hline $\mathrm{K}_{2} \mathrm{O}$ & 5.41 & 6.73 & 3.91 & 4.21 & 5.19 & 5.48 & 2.25 & 2.00 \\
\hline $\mathrm{P}_{2} \mathrm{O}_{5}$ & 0.09 & 0.02 & 0.23 & 0.20 & 0.17 & 0.13 & 0.52 & 0.52 \\
\hline Total & 99.55 & 99.71 & 100.10 & 99.76 & 99.57 & 100.16 & 99.58 & 99.80 \\
\hline $\mathrm{Rb}$ & 199.0 & 237.1 & 139.4 & 186.7 & 179.2 & 177.1 & 53.7 & 57.9 \\
\hline $\mathrm{Ba}$ & 675 & 126 & 732 & 935 & 960 & 911 & 787 & 1030 \\
\hline $\mathrm{Sr}$ & 130 & 60 & 218 & 209 & 169 & 145 & 987 & 993 \\
\hline $\mathrm{Pb}$ & 30 & 46 & 24 & 25 & 28 & 30 & 16 & 11 \\
\hline$T h^{\mathrm{c}}$ & 8.13 & 34.64 & 3.18 & 3.78 & 10.6 & 1.14 & 11.86 & $<0.59$ \\
\hline$U$ & 0.7 & 15.3 & 0.7 & 1.4 & n.d. & n.d. & 0.7 & $<0.63$ \\
\hline $\mathrm{Zr}$ & 147 & 75 & 396 & 369 & 295 & 215 & 338 & 188 \\
\hline$H f$ & 7.24 & 4.53 & 11.88 & 11.16 & 10.2 & 8.17 & 10.60 & 6.55 \\
\hline $\mathrm{Nb}$ & 7.7 & 3.9 & 13.1 & 10.5 & 8.9 & 6.9 & 9.7 & 6.7 \\
\hline$T a$ & 0.49 & 0.16 & 1.22 & 1.20 & 0.80 & 0.40 & 0.57 & 0.70 \\
\hline $\mathrm{Ni}$ & 4 & 1 & 5 & 4 & 5 & 5 & 24 & 32 \\
\hline $\mathrm{Zn}$ & 42 & 20 & 86 & 80 & 62 & 53 & 138 & 130 \\
\hline $\mathrm{Cr}$ & 23 & 19 & 46 & 35 & 31 & 28 & 95 & 144 \\
\hline $\mathrm{Ga}$ & 20 & 17 & 23 & 22 & 21 & 19 & 26 & 25 \\
\hline$S c$ & 6.474 & 3.19 & 15.80 & 13.16 & 9.68 & 6.64 & 17.83 & 20.24 \\
\hline V & 27 & 1 & 51 & 43 & 28 & 18 & 134 & 161 \\
\hline $\mathrm{La}$ & 54.3 & 81.7 & 49.0 & 48.1 & 59.5 & 58.0 & 84.6 & 39.2 \\
\hline $\mathrm{Ce}$ & 93.3 & 144.4 & 115.5 & 97.5 & 107.0 & 93.8 & 177.0 & 79.4 \\
\hline$N d$ & 38 & 58.7 & 58.1 & 52 & 51 & 42.5 & 85 & 47 \\
\hline$S m$ & 6.83 & 11.25 & 11.74 & 10.17 & 9.84 & 8.22 & 13.19 & 8.43 \\
\hline$E u$ & 1.27 & 0.52 & 2.03 & 1.84 & 1.75 & 1.59 & 3.40 & 2.66 \\
\hline$T b$ & 0.89 & 0.96 & 1.85 & 1.89 & 1.22 & 1.20 & 1.23 & 1.04 \\
\hline$Y b$ & 1.81 & 1.22 & 5.0 & 4.87 & 2.4 & 3.77 & 1.7 & 1.80 \\
\hline $\mathrm{Lu}$ & 0.24 & 0.15 & 0.69 & 0.71 & 0.26 & 0.58 & 0.23 & 0.28 \\
\hline $\mathrm{Y}$ & 19.1 & 10.6 & 56.6 & 48.7 & 25 & 32.5 & 23.4 & 21.8 \\
\hline $10^{4} \times \mathrm{Ga} / \mathrm{Al}$ & 2.65 & 2.54 & 2.74 & 2.68 & 2.70 & 2.51 & 2.76 & 2.86 \\
\hline $\mathrm{FeOt} /(\mathrm{FeOt}+\mathrm{MgO})$ & 0.85 & 0.98 & 0.81 & 0.84 & 0.86 & 0.86 & 0.68 & 0.64 \\
\hline A.S.I. (m) & 1.10 & 0.95 & 0.96 & 1.00 & 1.01 & 1.09 & 1.03 & 0.90 \\
\hline
\end{tabular}


Table 4 (Continued)

\begin{tabular}{|c|c|c|c|c|c|c|c|c|}
\hline $\begin{array}{l}\text { Map unit } \\
\text { sample }\end{array}$ & $\begin{array}{c}\text { Ycf } \\
\text { SNP-96-4A }\end{array}$ & $\begin{array}{c}\text { Ycf } \\
\text { SNP-97-20 }\end{array}$ & $\begin{array}{l}\text { Yfh } \\
\text { SNP-99-70 }\end{array}$ & $\begin{array}{l}\text { Yfh (z) } \\
\text { SNP-01-146 }\end{array}$ & $\begin{array}{c}\text { Yfpg } \\
\text { SNP-02-176 }\end{array}$ & $\begin{array}{l}\text { Yfpg (z) } \\
\text { SNP-02-177 }\end{array}$ & $\begin{array}{c}\text { Yfe (z) } \\
\text { SNP-03-204 }\end{array}$ & $\begin{array}{c}\text { Yfe } \\
\text { SNP-03-205 }\end{array}$ \\
\hline $\mathrm{SiO}_{2}{ }^{\mathrm{a}}$ & 71.80 & 69.48 & 69.92 & 69.69 & 67.83 & 67.36 & 66.88 & 67.44 \\
\hline $\mathrm{TiO}_{2}$ & 0.55 & 0.64 & 0.50 & 0.58 & 0.74 & 0.78 & 0.79 & 0.76 \\
\hline $\mathrm{Al}_{2} \mathrm{O}_{3}$ & 13.05 & 14.47 & 14.78 & 15.21 & 14.97 & 14.73 & 14.55 & 14.62 \\
\hline $\mathrm{Fe}_{2} \mathrm{O}_{3} \mathrm{~b}$ & 3.48 & 4.04 & 3.07 & 3.80 & 4.86 & 5.34 & 6.19 & 6.03 \\
\hline $\mathrm{MnO}$ & 0.06 & 0.07 & 0.04 & 0.05 & 0.06 & 0.08 & 0.10 & 0.10 \\
\hline $\mathrm{MgO}$ & 0.49 & 0.43 & 0.92 & 0.68 & 0.90 & 0.95 & 0.98 & 0.90 \\
\hline $\mathrm{CaO}$ & 1.71 & 2.26 & 2.16 & 1.87 & 2.73 & 2.72 & 2.76 & 2.75 \\
\hline $\mathrm{Na}_{2} \mathrm{O}$ & 2.45 & 2.52 & 3.53 & 2.34 & 2.71 & 2.73 & 2.58 & 2.64 \\
\hline $\mathrm{K}_{2} \mathrm{O}$ & 5.87 & 5.68 & 4.21 & 6.13 & 4.85 & 4.83 & 4.90 & 4.85 \\
\hline $\mathrm{P}_{2} \mathrm{O}_{5}$ & 0.11 & 0.16 & 0.18 & 0.15 & 0.19 & 0.22 & 0.24 & 0.22 \\
\hline Total & 99.56 & 99.75 & 99.29 & 100.49 & 99.84 & 99.74 & 99.96 & 100.31 \\
\hline $\mathrm{Rb}$ & 270.5 & 187.8 & 120 & 166.7 & 191.9 & 186.2 & 153.8 & 164.4 \\
\hline $\mathrm{Ba}$ & 424 & 806 & 841 & 951 & 841 & 863 & 1034 & 977 \\
\hline $\mathrm{Sr}$ & 79 & 118 & 363 & 170 & 165 & 161 & 171 & 176 \\
\hline $\mathrm{Pb}$ & 32 & 27 & 23 & 28 & 28 & 28 & 24 & 28 \\
\hline$T h^{\mathrm{c}}$ & 6.0 & 2.3 & 17.9 & 3.19 & 6.5 & 15.00 & 0.38 & 2.03 \\
\hline$U$ & 0.6 & 0.6 & 2.18 & n.d. & 0.7 & 1.5 & 0.7 & 0.9 \\
\hline $\mathrm{Zr}$ & 271 & 264 & 187 & 239 & 277 & 336 & 308 & 315 \\
\hline$H f$ & 8.1 & 10.1 & 5.63 & 9.26 & 11.16 & 13.07 & 11.61 & 11.21 \\
\hline $\mathrm{Nb}$ & 7.4 & 7.7 & 8.8 & 8.6 & 8.9 & 10.4 & 14.3 & 12.8 \\
\hline$T a$ & 0.46 & 0.87 & 0.64 & 0.50 & 1.10 & 1.10 & 0.98 & 1.16 \\
\hline $\mathrm{Ni}$ & 3 & 3 & 7 & 5 & 6 & 8 & 8 & 7 \\
\hline $\mathrm{Zn}$ & 49 & 71 & 53 & 64 & 68 & 77 & 105 & 110 \\
\hline $\mathrm{Cr}$ & 33 & 28 & 36 & 27 & 34 & 39 & 40 & 33 \\
\hline $\mathrm{Ga}$ & 21 & 21 & 19 & 20 & 22 & 22 & 22 & 22 \\
\hline$S c$ & 6.88 & 8.37 & 7.29 & 10.84 & 11.88 & 12.57 & 16.12 & 16.04 \\
\hline $\mathrm{V}$ & 21 & 25 & 33 & 33 & 44 & 49 & 45 & 40 \\
\hline$L a$ & 55.3 & 48.4 & 41 & 46.2 & 55.8 & 69.5 & 43.0 & 66.0 \\
\hline $\mathrm{Ce}$ & 112.4 & 86.3 & 94.5 & 100.4 & 107.5 & 129.2 & 91.1 & 111.5 \\
\hline$N d$ & 52 & 41 & 39.1 & 42.9 & 49 & 61 & 46.4 & 55.8 \\
\hline$S m$ & 12.72 & 9.16 & 8.6 & 7.06 & 9.56 & 10.82 & 10.08 & 12.52 \\
\hline$E u$ & 1.35 & 1.93 & 1.49 & 1.83 & 1.76 & 1.72 & 2.16 & 2.27 \\
\hline$T b$ & 1.50 & 1.43 & 0.99 & 0.85 & 0.00 & 0.00 & 1.58 & 1.65 \\
\hline$Y b$ & 4.3 & 3.8 & 1.92 & 1.9 & 3.87 & 3.9 & 4.74 & 5.53 \\
\hline$L u$ & 0.58 & 0.43 & 0.36 & 0.27 & 0.54 & 0.57 & 0.74 & 0.87 \\
\hline $\mathrm{Y}$ & 48 & 41.5 & 26.5 & 20.5 & 39.5 & 40.1 & 45.8 & 48.9 \\
\hline $10^{4} \times \mathrm{Ga} / \mathrm{Al}$ & 3.04 & 2.74 & 2.43 & 2.48 & 2.78 & 2.82 & 2.86 & 2.84 \\
\hline $\mathrm{FeOt} /(\mathrm{FeOt}+\mathrm{MgO})$ & 0.86 & 0.90 & 0.75 & 0.83 & 0.83 & 0.84 & 0.85 & 0.86 \\
\hline A.S.I. (m) & 0.98 & 1.02 & 1.05 & 1.11 & 1.04 & 1.03 & 1.08 & 1.02 \\
\hline
\end{tabular}


Table 4 (Continued)

\begin{tabular}{|c|c|c|c|c|c|c|c|c|}
\hline $\begin{array}{l}\text { Map unit } \\
\text { sample }\end{array}$ & $\begin{array}{c}\text { Ylgg } \\
\text { SNP-99-90A }\end{array}$ & $\begin{array}{l}\text { Ylgg (z) } \\
\text { SNP-99-90B }\end{array}$ & $\begin{array}{l}\text { Ygg (z) } \\
\text { SNP-01-170 }\end{array}$ & $\begin{array}{l}\text { Ygg (z) } \\
\text { SNP-03-197 }\end{array}$ & $\begin{array}{l}\text { Ygr } \\
\text { OR-97-36 }\end{array}$ & $\begin{array}{l}\text { Ygr } \\
\text { OR-97-59 }\end{array}$ & $\begin{array}{c}\text { Yfqj } \\
\text { SNP-00-121 }\end{array}$ & $\begin{array}{c}\text { Yfqj } \\
\text { SNP-03-200 }\end{array}$ \\
\hline $\mathrm{SiO}_{2}{ }^{\mathrm{a}}$ & 75.35 & 74.26 & 75.18 & 64.10 & 73.64 & 71.89 & 62.37 & 59.27 \\
\hline $\mathrm{TiO}_{2}$ & 0.04 & 0.20 & 0.28 & 0.23 & 0.24 & 0.45 & 1.83 & 1.94 \\
\hline $\mathrm{Al}_{2} \mathrm{O}_{3}$ & 13.74 & 13.93 & 12.84 & 18.44 & 13.88 & 14.23 & 13.90 & 14.77 \\
\hline $\mathrm{Fe}_{2} \mathrm{O}_{3} \mathrm{~b}$ & 0.47 & 1.94 & 2.16 & 6.59 & 1.86 & 2.87 & 8.04 & 9.53 \\
\hline $\mathrm{MnO}$ & 0.01 & 0.02 & 0.04 & 0.26 & 0.05 & 0.06 & 0.11 & 0.13 \\
\hline $\mathrm{MgO}$ & 0.02 & 0.22 & 0.14 & 0.28 & 0.24 & 0.48 & 1.54 & 1.30 \\
\hline $\mathrm{CaO}$ & 0.36 & 0.79 & 0.94 & 2.92 & 1.08 & 1.54 & 4.10 & 5.26 \\
\hline $\mathrm{Na}_{2} \mathrm{O}$ & 2.59 & 3.15 & 2.27 & 4.13 & 2.49 & 2.44 & 2.69 & 2.79 \\
\hline $\mathrm{K}_{2} \mathrm{O}$ & 7.35 & 6.16 & 5.71 & 2.71 & 6.16 & 6.08 & 4.43 & 3.53 \\
\hline $\mathrm{P}_{2} \mathrm{O}_{5}$ & 0.02 & 0.04 & 0.06 & 0.11 & 0.06 & 0.13 & 0.81 & 0.98 \\
\hline Total & 99.95 & 100.71 & 99.61 & 99.77 & 99.70 & 100.16 & 99.83 & 99.50 \\
\hline $\mathrm{Rb}$ & 177.4 & 161.5 & 229.3 & 65.9 & 272.1 & 245.1 & 139 & 101.7 \\
\hline $\mathrm{Ba}$ & 1302 & 1009 & 638 & 786 & 350 & 777 & 1281 & 1553 \\
\hline $\mathrm{Sr}$ & 111 & 157 & 115 & 511 & 76 & 248 & 383 & 510 \\
\hline $\mathrm{Pb}$ & 32 & 28 & 60 & 39 & 57 & 44 & 25 & 29 \\
\hline$T h^{\mathrm{c}}$ & 9.05 & 33.4 & 23.42 & 38.20 & 53.6 & 35.1 & 26.8 & 2.83 \\
\hline$U$ & 0.04 & 1.2 & 2.8 & 3.2 & 9.6 & 0.0 & 7.8 & 0.9 \\
\hline $\mathrm{Zr}$ & 31 & 169 & 222 & 331 & 148 & 216 & 839 & 866 \\
\hline$H f$ & 1.5 & 6.8 & 9.45 & 10.38 & 7.3 & 4.7 & 4.2 & 27.37 \\
\hline $\mathrm{Nb}$ & 0.5 & 6.7 & 6.0 & 7.8 & 5 & 7 & 26.5 & 28.9 \\
\hline $\mathrm{Ta}$ & 0.08 & 0.72 & 0.32 & 0.42 & 0.45 & 0.50 & 0.18 & 1.74 \\
\hline $\mathrm{Ni}$ & 2 & 4 & 3 & 1 & 2 & 2 & 3 & 3 \\
\hline $\mathrm{Zn}$ & 5 & 18 & 62 & 41 & 41 & 61 & 193 & 226 \\
\hline $\mathrm{Cr}$ & 20 & 30 & 23 & 20 & 31 & 23 & 22 & 31 \\
\hline $\mathrm{Ga}$ & 19 & 22 & 20 & 27 & 23 & 22 & 26 & 29 \\
\hline$S c$ & 0.64 & 3.75 & 6.18 & 5.04 & 3.95 & 5.01 & 2.437 & 18.41 \\
\hline V & 2 & 5 & 7 & 1 & 6 & 14 & 64 & 64 \\
\hline $\mathrm{La}$ & 64.3 & 94 & 67.7 & 103.2 & 75.2 & 73.0 & 97 & 99.8 \\
\hline $\mathrm{Ce}$ & 93.1 & 190.0 & 147.4 & 323.4 & 130.0 & 141.0 & 133.8 & 252.0 \\
\hline$N d$ & 25 & 64 & 64 & 121.4 & 50 & 53 & 52 & 142.1 \\
\hline$S m$ & 2.78 & 8.35 & 10.92 & 16.83 & 11.10 & 12.00 & 10.49 & 27.53 \\
\hline$E u$ & 1.27 & 1.65 & 1.09 & 2.02 & 0.95 & 1.95 & 0.921 & 4.65 \\
\hline$T b$ & 0.18 & 0.62 & 1.58 & 1.65 & 1.29 & 1.10 & 0.76 & 2.07 \\
\hline$Y b$ & 0.54 & 1.38 & 6.0 & 5.21 & 5.3 & 2.9 & 0.99 & 7.09 \\
\hline$L u$ & 0.08 & 0.2 & 0.87 & 0.79 & 0.61 & 0.22 & 0.13 & 1.03 \\
\hline $\mathrm{Y}$ & 2.8 & 8.1 & 45.1 & 38.8 & 27.8 & 33.7 & 103.4 & 86.5 \\
\hline $10^{4} \times \mathrm{Ga} / \mathrm{Al}$ & 2.61 & 2.98 & 2.94 & 2.77 & 3.13 & 2.92 & 3.53 & 3.71 \\
\hline $\mathrm{FeOt} /(\mathrm{FeOt}+\mathrm{MgO})$ & 0.96 & 0.89 & 0.93 & 0.95 & 0.88 & 0.84 & 0.82 & 0.87 \\
\hline A.S.I. (m) & 1.07 & 1.05 & 1.11 & 1.24 & 1.10 & 1.07 & 0.89 & 0.88 \\
\hline
\end{tabular}




\begin{tabular}{|c|c|c|c|c|}
\hline $\begin{array}{l}\text { Map unit } \\
\text { sample }\end{array}$ & $\begin{array}{l}\text { Ybg } \\
\text { OR-97-65 }\end{array}$ & $\begin{array}{c}\text { Ybg (z) } \\
\text { SNP-02-186 }\end{array}$ & $\begin{array}{c}\text { USGS G-2 } \\
\text { mean }^{\mathrm{d}}\end{array}$ & $\begin{array}{l}\text { USGS G-2 } \\
\text { average dev }\end{array}$ \\
\hline $\mathrm{SiO}_{2}{ }^{\mathrm{a}}$ & 67.06 & 62.41 & 70.14 & 0.225 \\
\hline $\mathrm{TiO}_{2}$ & 0.75 & 1.44 & 0.49 & 0.005 \\
\hline $\mathrm{Al}_{2} \mathrm{O}_{3}$ & 15.11 & 13.87 & 15.40 & 0.088 \\
\hline $\mathrm{Fe}_{2} \mathrm{O}_{3}{ }^{\mathrm{b}}$ & 4.91 & 9.13 & 2.64 & 0.009 \\
\hline $\mathrm{MnO}$ & 0.07 & 0.15 & 0.04 & 0.003 \\
\hline $\mathrm{MgO}$ & 0.96 & 0.81 & 0.70 & 0.033 \\
\hline $\mathrm{CaO}$ & 2.62 & 3.97 & 1.94 & 0.006 \\
\hline $\mathrm{Na}_{2} \mathrm{O}$ & 2.89 & 2.80 & 4.08 & 0.079 \\
\hline $\mathrm{K}_{2} \mathrm{O}$ & 5.05 & 4.43 & 4.50 & 0.018 \\
\hline $\mathrm{P}_{2} \mathrm{O}_{5}$ & 0.33 & 0.69 & 0.14 & 0.002 \\
\hline Total & 99.75 & 99.70 & 100.06 & \\
\hline $\mathrm{Rb}$ & 199.9 & 109.1 & 169.4 & 0.24 \\
\hline $\mathrm{Ba}$ & 929 & 2112 & 1874.8 & 7.04 \\
\hline $\mathrm{Sr}$ & 266 & 314 & 483.6 & 1.12 \\
\hline $\mathrm{Pb}$ & 40 & 28 & 30.6 & 0.48 \\
\hline$T h^{\mathrm{c}}$ & 31.7 & 1.40 & & \\
\hline$U$ & 3.2 & $<0.83$ & & \\
\hline $\mathrm{Zr}$ & 561 & 1354 & 321.6 & 3.28 \\
\hline$H f$ & 19.6 & 43 & & \\
\hline $\mathrm{Nb}$ & 13 & 25.2 & 11.8 & 0.25 \\
\hline$T a$ & 0.70 & 1.40 & & \\
\hline $\mathrm{Ni}$ & 3 & 1 & 2.6 & 0.48 \\
\hline $\mathrm{Zn}$ & 106 & 249 & 92.6 & 0.48 \\
\hline $\mathrm{Cr}$ & 27 & 11 & 7.8 & 0.32 \\
\hline $\mathrm{Ga}$ & 26 & 25 & 22.0 & 0.0 \\
\hline$S c$ & 7.31 & 20.0 & & \\
\hline $\mathrm{V}$ & 31 & 39 & 33.2 & 0.64 \\
\hline$L a$ & 112.7 & 83.6 & & \\
\hline $\mathrm{Ce}$ & 235.2 & 170.0 & & \\
\hline$N d$ & 91 & 106 & & \\
\hline$S m$ & 15.35 & 18.87 & & \\
\hline$E u$ & 2.195 & 5.0 & & \\
\hline$T b$ & 1.34 & 1.90 & & \\
\hline$Y b$ & 1.85 & 4.6 & & \\
\hline$L u$ & 0.26 & 0.73 & & \\
\hline $\mathrm{Y}$ & 23.1 & 58.6 & 7.9 & 0.62 \\
\hline $10^{4} \times \mathrm{Ga} / \mathrm{Al}$ & 3.25 & 3.41 & & \\
\hline $\mathrm{FeOt} /(\mathrm{FeOt}+\mathrm{MgO})$ & 0.82 & 0.91 & & \\
\hline A.S.I. (m) & 1.04 & 0.88 & & \\
\hline
\end{tabular}

A.S.I. $(m)$ (modified aluminum saturation index) $=$ molar $\left.\mathrm{Al}_{2} \mathrm{O}_{3} /\left[\left(\mathrm{CaO}-1.67 \times \mathrm{P}_{2} \mathrm{O}_{5}\right)+\mathrm{Na}_{2} \mathrm{O}+\mathrm{K}_{2} \mathrm{O}\right]\right)$, (z) zircon analyzed for geochronology (this study); n.d.: not detected.

${ }^{\text {a }}$ Major elements expressed in weight percent; trace elements in parts per million.

b Total iron expressed as $\mathrm{Fe}_{2} \mathrm{O}_{3}$.

${ }^{c}$ Elements in italics were analyzed by neutron activation; others by X-ray fluorescence.

d Mean of 103 (majors) and 5 (traces) replicate analyses of USGS G-2 standard.

e Average deviation from mean. 

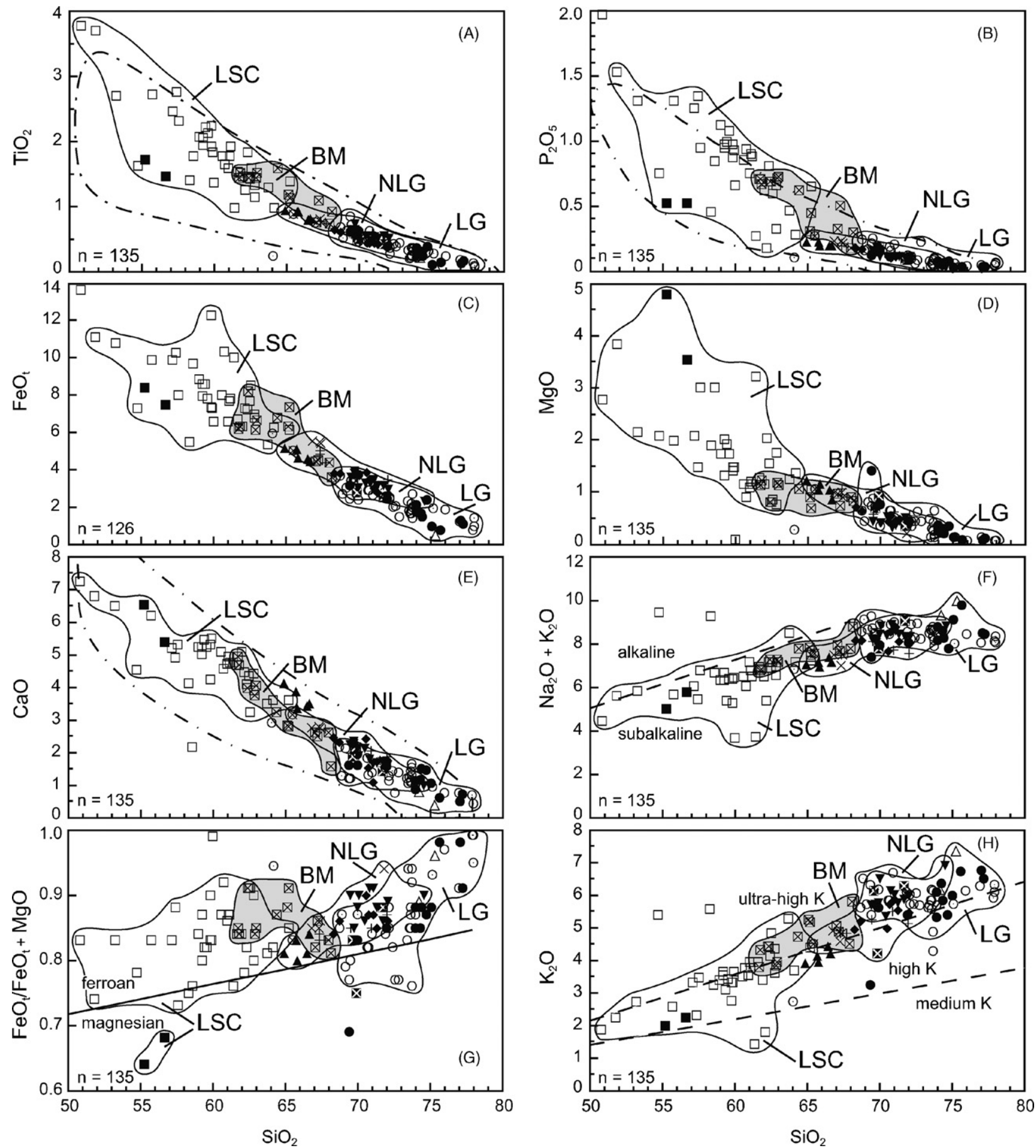

Fig. 9. Plots of $\mathrm{SiO}_{2}$ vs. $\mathrm{TiO}_{2}$ (A), $\mathrm{P}_{2} \mathrm{O}_{5}$ (B), $\mathrm{FeOt}(\mathrm{C}), \mathrm{MgO}$ (D), $\mathrm{CaO}(\mathrm{E}), \mathrm{Na}_{2} \mathrm{O}+\mathrm{K}_{2} \mathrm{O}(\mathrm{F}), \mathrm{FeOt} / \mathrm{FeOt}+\mathrm{MgO}(\mathrm{G})$, and $\mathrm{K}_{2} \mathrm{O}$ (H) for rocks of the study area. All data are expressed in weight percent. Symbols are the same as in Fig. 4. Data fields for lithologies include: LSC, low-silica charnockites (Ypqd and Yfqj); BM, biotite-rich monzogranite (Ybg); NLG: non-leucocratic granitoids (Ylgn, Yg, Ycf, Yfh, Yfe, Yfpg); and LG, leucogranites (Ylg, Ylgg, Ygg, Ygr). FeOt refers to total iron expressed as FeO. Dash-dot lines in A, B, and E outline compositional fields of charnockitic rocks of igneous origin from Kilpatrick and Ellis (1992). Boundary separating ferroan (tholeiitic) and magnesian (calc-alkaline) fields in $\mathrm{E}$ is from Frost et al. (2001). Dashed lines separating medium-K, high-K, and ultra-K fields in H are modified from Gill (1981). 

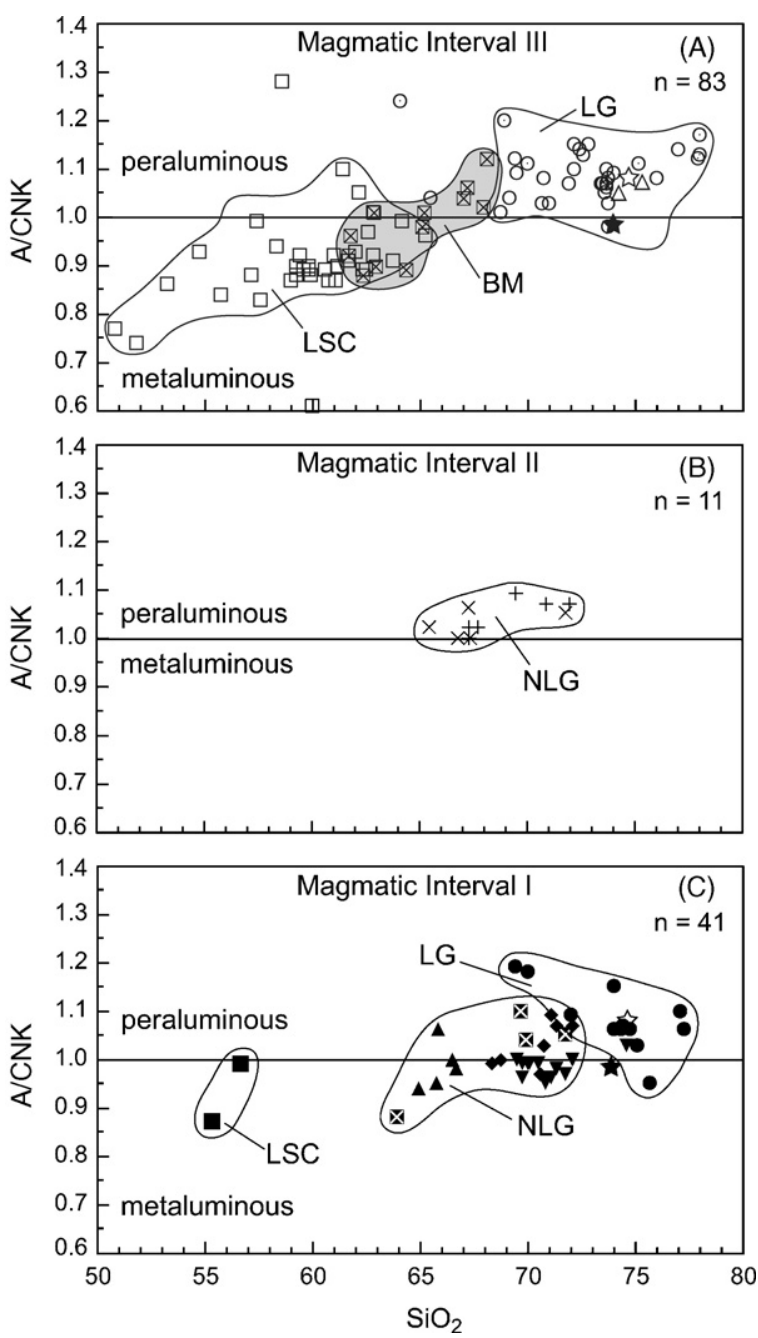

Fig. 10. Plots of $\mathrm{SiO}_{2}$ vs. modified aluminum saturation index $\left(\mathrm{A} / \mathrm{CNK}=\right.$ molar $\left.\mathrm{Al}_{2} \mathrm{O}_{3} /\left[\left(\mathrm{CaO}-1.67 \times \mathrm{P}_{2} \mathrm{O}_{5}\right)+\mathrm{Na}_{2} \mathrm{O}+\mathrm{K}_{2} \mathrm{O}\right]\right)$ for rocks emplaced during Magmatic Intervals III (A), II (B), and I (C) separated by lithology. Symbols are the same as in Fig. 4. Data fields for lithologies include: LSC, low-silica charnockites (Ypqd (Magmatic Interval I) and Yfqj (III)); BM, biotite-rich monzogranite (Ybg (III)); NLG: non-leucocratic granitoids (Ylgn, Yg, Ycf, Yfh (I), and Yfe, Yfpg (II)); and LG, leucogranites (Ylg (I) and Ylgg, Ygg, Ygr (III)). Filled and unfilled stars in $\mathrm{A}$ and $\mathrm{C}$ indicate the compositions of melts produced from tonalite in the experiments of Patiño-Douce (1997) at 4 and $8 \mathrm{kbar}$, respectively.

trations and ratios that bear directly on their igneous origin (Fig. 11). The low-silica charnockites, characterized by low Rb concentrations (Fig. 11A), represent the chemically least evolved rocks. In contrast, the higher silica granitoids, especially the leucogranites, exhibit relatively evolved compositions, including characteristics such as low $\mathrm{Sr}$ concentrations and high $\mathrm{Rb} / \mathrm{Ba}$ and $\mathrm{K} / \mathrm{Ba}$ ratios (Fig. 11B and D) that likely result from feldspar fractionation. Compositional distinctions between rock types are further supported by differences in concentrations of source-sensitive, compatible, high-field-strength elements (HFSE) such as $\mathrm{Nb}, \mathrm{Zr}, \mathrm{Ta}$, and $\mathrm{Y}$ that are generally higher in the low-silica charnockites and lower in the higher silica granitoids (Fig. 11E-H). Significantly, HFSE concentrations illuminate differences between the two low-silica charnockites; 1160 Ma pyroxene quartz diorite (Ypqd) consistently displays lower concentrations than ca. $1050 \mathrm{Ma}$ farsundite-quartz jotunite (Yfqj). The biotite-rich monzogranite (Ybg) is compositionally transitional between Yfqj and the higher silica rocks. Elemental concentrations in samples of low-silica charnockite (Yfqj) and biotite-rich monzogranite (Ybg) of Magmatic Interval III show the greatest variation in HFSE because of internal fractionation and inclusion of dikes (Yfqj), large-scale compositional layering (Ybg), and local alteration. Y concentrations in leucogranites $\mathrm{Ygr}$ and Ygg are generally low; however, high values in some samples are probably caused by local accumulation of garnet.

\section{Nd isotopic characteristics}

Twenty-one samples were analyzed for $\mathrm{Nd}$ and $\mathrm{Sm}$ concentrations and $\mathrm{Nd}$ isotopic compositions. Data for representative samples from each lithologic unit are presented in Table 5 and include 13 of the 14 samples for which crystallization ages are reported in Table 3.

\subsection{Analytical methods}

$\mathrm{Sm}-\mathrm{Nd}$ analysis followed procedures described in Holmden and Dickin (1995). After dissolution using HF and $\mathrm{HNO}_{3}$, samples were split and one aliquot spiked with a mixed ${ }^{150} \mathrm{Nd}-{ }^{149} \mathrm{Sm}$ spike. Standard cation and reverse phase column separation methods were used. $\mathrm{Nd}$ isotope analyses were performed on a VG isomass 354 mass spectrometer at McMaster University using double filaments and a four collector peak switching algorithm, and were normalized to a ${ }^{146} \mathrm{Nd} /{ }^{144} \mathrm{Nd}$ ratio of 0.7219 . An average value of $0.51185 \pm 2$ (2-sigma) was determined for the La Jolla standard. Analysis by this technique yielded $\mathrm{Sm} / \mathrm{Nd}=0.2280 \pm 2$ for U.S. Geological Survey standard BCR-1. Average withinrun precision for samples was \pm 0.000012 (2 sigma). Reproducibilities of model ages and initial $\mathrm{Nd}$ ratios are estimated at \pm 20 m.y. and \pm 0.4 epsilon units, respectively ( 2 sigma), based on experience analyzing duplicate dissolutions. 

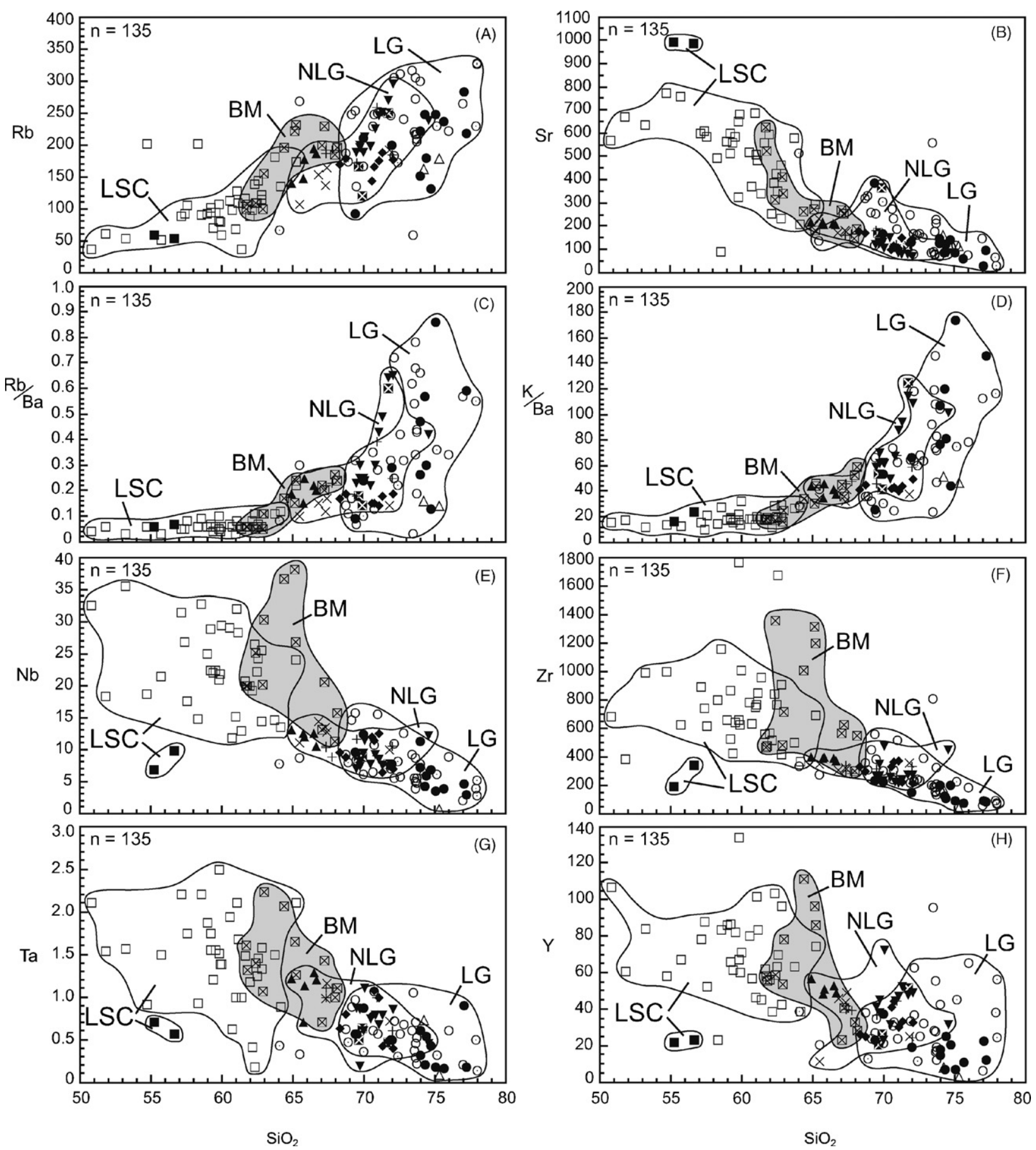

Fig. 11. Plots of $\mathrm{SiO}_{2}$ vs. $\mathrm{Rb}(\mathrm{A}), \mathrm{Sr}(\mathrm{B}), \mathrm{Rb} / \mathrm{Ba}(\mathrm{C}), \mathrm{K} / \mathrm{Ba}(\mathrm{D}), \mathrm{Nb}(\mathrm{E}), \mathrm{Zr}(\mathrm{F}), \mathrm{Ta}(\mathrm{G})$, and $\mathrm{Y}(\mathrm{H})$ for rocks of the study area. All elemental data are expressed in parts per million. Symbols are the same as in Fig. 4. Labels of data fields for lithologies are the same as in Fig. 9.

\subsection{Nd isotope results}

$\mathrm{Nd}$ isotope compositions and $\mathrm{Sm}-\mathrm{Nd}$ concentration data (Table 5) were used to calculate a $T_{\mathrm{dm}}$ model age for each sample using the depleted mantle model of DePaolo
(1981). Model ages (significantly older than zircon crystallization ages for all samples) range from 1.37 to $1.62 \mathrm{Ga}$, indicating that none of the samples are juvenile. The granitoids must therefore have been derived by melting of older enriched sources such as continental crust. 
Table 5

$\mathrm{Sm}-\mathrm{Nd}$ isotopic data for Grenvillian rocks of the northern Virginia Blue Ridge

\begin{tabular}{|c|c|c|c|c|c|c|c|c|}
\hline Sample & Unit & Igneous crystallization age (Ma) & $\mathrm{Nd}(\mathrm{ppm})$ & $\mathrm{Sm}(\mathrm{ppm})$ & ${ }^{147} \mathrm{Sm} /{ }^{144} \mathrm{Nd}$ & ${ }^{143} \mathrm{Nd} /{ }^{144} \mathrm{Nd}$ & $\varepsilon_{\mathrm{Nd}}$ & $T_{\mathrm{DM}}(\mathrm{Ga})$ \\
\hline \multicolumn{9}{|c|}{ Magmatic Interval III } \\
\hline SNP-02-186 & Ybg & $1028 \pm 9$ & 108 & 20.9 & 0.1171 & 0.512106 & 0.0 & 1.59 \\
\hline SNP-99-93 & Yfqj & $1050 \pm 8^{\mathrm{a}}$ & 144 & 27.4 & 0.1146 & 0.512075 & 0.0 & 1.49 \\
\hline SNP-99-89 & Yfqj & & 161 & 32.1 & 0.1206 & 0.512119 & 0.1 & 1.51 \\
\hline SNP-03-200 & Yfqj & & 146 & 28.1 & 0.1163 & 0.512103 & 0.3 & 1.47 \\
\hline OR-97-35 & Ygr & $1060 \pm 5^{\mathrm{a}}$ & 88.8 & 18.35 & 0.1248 & 0.512117 & -0.5 & 1.58 \\
\hline SNP-02-197 & Ygg & $1063 \pm 8$ & 144.5 & 18.4 & 0.0770 & 0.511769 & -0.7 & 1.42 \\
\hline SNP-01-170 & Ygg & & 55.5 & 10.0 & 0.1087 & 0.512012 & -0.3 & 1.49 \\
\hline SNP-99-90 & Ylgg & $1078 \pm 9^{\mathrm{a}}$ & 60.4 & 7.89 & 0.0789 & 0.511836 & 0.5 & 1.37 \\
\hline \multicolumn{9}{|c|}{ Magmatic Interval II } \\
\hline SNP-03-204 & Yfe & $1111 \pm 16$ & 47.3 & 9.85 & 0.1258 & 0.512108 & -0.3 & 1.62 \\
\hline SNP-02-177 & Yfpg & $1120 \pm 12$ & 58.8 & 10.9 & 0.1121 & 0.512020 & 0.0 & 1.53 \\
\hline \multicolumn{9}{|c|}{ Magmatic Interval I } \\
\hline SNP-01-146 & Yfh & $1144 \pm 8$ & 39.3 & 7.18 & 0.1105 & 0.512002 & 0.2 & 1.54 \\
\hline SNP-96-10 & Ycf & $1159 \pm 14^{\mathrm{a}}$ & 52.5 & 10.4 & 0.1193 & 0.512047 & -0.1 & 1.61 \\
\hline SNP-96-4 & Ycf & & 66 & 12.9 & 0.1184 & 0.512107 & 1.2 & 1.49 \\
\hline SNP-02-175 & Ypqd & $1161 \pm 10$ & 46.4 & 8.80 & 0.1146 & 0.512136 & 2.4 & 1.39 \\
\hline SNP-01-154 & Ypqd & & 81.3 & 13.2 & 0.0979 & 0.511980 & 1.9 & 1.40 \\
\hline SNP-03-198 & $\mathrm{Yg}$ & ca. 1180 & 39.4 & 7.41 & 0.1138 & 0.512039 & 0.8 & 1.53 \\
\hline SNP-01-150 & Ylgn & $1165 \pm 7$ & 51.8 & 11.0 & 0.1284 & 0.512159 & 0.8 & 1.58 \\
\hline SNP-02-184 & Ylgn & & 51.2 & 10.7 & 0.1261 & 0.512143 & 0.8 & 1.56 \\
\hline SNP-03-199 & Ylg2 & $1175 \pm 11$ & 49.9 & 9.09 & 0.1101 & 0.512031 & 1.1 & 1.49 \\
\hline SNP-02-189 & Ylg1 & $1183 \pm 11$ & 38.4 & 6.82 & 0.1074 & 0.511977 & 0.5 & 1.53 \\
\hline SNP-02-193 & Ylg1 & & 49.6 & 9.60 & 0.1168 & 0.512071 & 1.0 & 1.53 \\
\hline
\end{tabular}

a Ages from Tollo et al. (2004a).

$\mathrm{U}-\mathrm{Pb}$ ages for each unit were used to calculate initial $\mathrm{Nd}$ isotope ratios at the time of crystallization in the form of $\varepsilon_{\mathrm{Nd}(t)}$ (part per 10,000 deviation from Bulk Earth at time $t$ ) (Table 5). Plotted in terms of $\mathrm{Nd}$ isotope evolution against time (Fig. 12), all values fall well below the depleted mantle $\left(T_{\mathrm{dm}}\right)$ growth curve, fur-

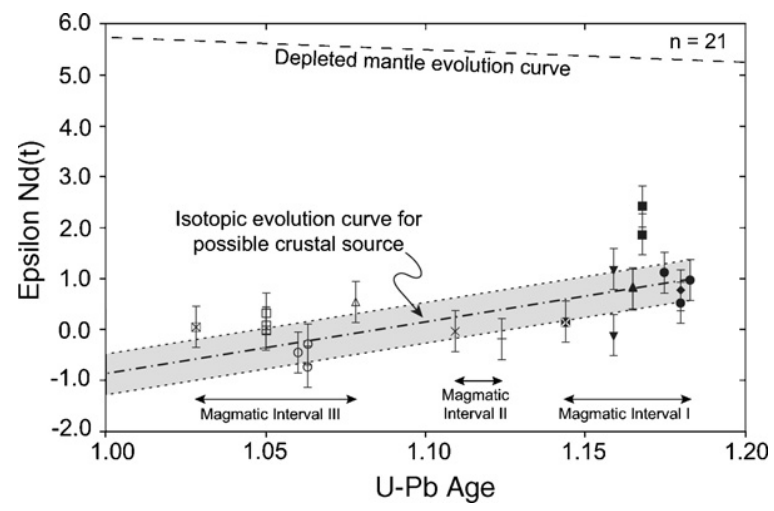

Fig. 12. Plot of Nd isotope evolution against time, showing initial ratios for analyzed units relative to evolution curves for the depleted mantle (dashed line, after DePaolo (1981)) and for the oldest unit in the study area (Ylg) (dash-dot line). Symbols are the same as in Fig. 4. Error bars are estimated analytical reproducibilites (2 sigma). Shaded field represents 2-sigma isotopic evolution of lithologic unit Ylg. ther indicating that sources were enriched relative to depleted mantle. Most samples lie relatively close to a Bulk Earth evolution line $\left(\varepsilon_{\mathrm{Nd}}=0\right)$, with a small range from -0.7 to +1.2 indicating that they are unlikely to be derived from a primordial undepleted mantle source. Instead, the magmas were most likely produced by melting of an old crustal source. The Nd evolution of the Ylg unit is considered an approximation of crustally derived parental magmas that gave rise to most lithologic units because this pluton was among the first to be emplaced and is compositionally similar to magmatic sources inferred in the central Virginia Blue Ridge (Hughes et al., 2004). This source has a present-day value $\left(\varepsilon_{0}\right)$ of -11.1 . Two samples of unit Ypqd have $\varepsilon_{\mathrm{Nd}(t)}$ values of +1.9 and +2.4 , significantly different from the rest of the suite. These rocks probably sampled a slightly more primitive source but are nonetheless not mantle derived. Fullagar et al. (1997) likewise demonstrated that Grenville-age rocks from the southern Appalachians were derived from recycled Laurentian crust of Middle Proterozoic age. Pettingill et al. (1984) and Owens and Samson (2004) reported initial $\varepsilon_{\mathrm{Nd}}$ values and depleted mantle model ages $\left(T_{\mathrm{dm}}\right)$ from Grenville-age rocks elsewhere in Virginia that are similar to the rocks of this study. 


\section{Petrogenesis}

Mesoproterozoic rocks of the Virginia Blue Ridge constitute a granitoid-charnockite association that is comparable to other suites worldwide (e.g. Emslie et al., 1994; Vander Auwera et al., 2003). The occurrence of minor anorthosite of circa $1045 \mathrm{Ma}$ age (Pettingill et al., 1984) further suggests that Blue Ridge crust has much in common with anorthosite-mangerite-charnockitegranite (AMCG) suites that are a widespread component of Earth's Proterozoic orogens (Emslie, 1978). The dominance of granitoids in the Blue Ridge suggests that the present erosion level is more shallow than in areas such Labrador and the Grenville Province of Canada, where granitic rocks and anorthosites occur in more equal proportions (Emslie et al., 1994).

\subsection{Petrogenetic relationships}

The petrogenesis of charnockitic rocks has received considerable attention in recent decades as researchers have documented the igneous heritage of many opxbearing suites. In the Virginia Blue Ridge, the close proximity of both charnockitic and non-charnockitic rocks of similar age and likely igneous parentage suggests that the presence of opx results from intrinsic characteristics of the original magmas and therefore, in most cases, is not a direct manifestation of metamorphic recrystallization. Similar juxtaposition of opx-bearing and opx-free igneous rocks of granitic composition was observed by Frost et al. (2000) in the $2.6 \mathrm{Ga}$ Louis Lake batholith in Wyoming where the occurrence of pyroxene was restricted to high pressure zones where $\mathrm{CO}_{2}$ was enriched in magmatic fluids at the expense of $\mathrm{H}_{2} \mathrm{O}$. Liogys and Tracy (2004) documented igneous temperatures of equilibation $\left(920-980^{\circ} \mathrm{C}\right)$ for coexisting pyroxenes in charnockitic rocks from the Blue Ridge, providing additional evidence for magmatic origin.

The extended compositional range characterizing Blue Ridge granitoids emplaced during Magmatic Intervals I and III suggests that the rocks preserve evidence of differentiation processes. In each case the relatively unevolved low-silica charnockites could represent an approximation of magmas parental to contemporaneous higher silica rocks. However, for Magmatic Interval I, the broad compositional gap between low-silica magnesian charnockite (Ypqd) and associated, exclusively tholeiitic granitoids (Fig. 9) precludes a cogenetic relationship, especially fractionation. Among the higher silica rocks, crystallization ages of most non-leucocratic granitoids are statistically irresolvable from the age range of associated Ylg leucogranites (Table 3), suggesting that a petrogenetic relationship is possible. Moreover, the leucogranites display compositional features consistent with greater fractionation, as illustrated by higher $\mathrm{SiO}_{2}$, $\mathrm{La} / \mathrm{Yb}, \mathrm{Rb} / \mathrm{Ba}$, and $\mathrm{Rb} / \mathrm{Sr}$ (Table 4; Figs. 9 and 11), in addition to lower Eu/Eu ${ }^{*}$ (ratio of measured Eu concentration divided by the value derived through interpolation of adjacent REE) in some samples (Tollo et al., 2004a). However, considerable overlap in data suggest that it is unlikely that the non-leucocratic granitoids fractionated to produce the leucogranite magmas. The preponderance of data implies that leucogranites and non-leucocratic granitoids of Magmatic Interval I represent products of fractionation processes in which removal of feldspar was important; however, rocks representing parental magmas have not been recognized.

The considerable range in $\mathrm{SiO}_{2}, \mathrm{FeOt} /(\mathrm{FeOt}+\mathrm{MgO})$, and total alkali content of granitoids emplaced during Magmatic Interval III (Fig. 9) could define a cogenetic association in which low-silica charnockite (Yfqj) is an approximation of parental magma that ultimately produced penecontemporaneous leucogranites. Low Sr concentrations and elevated $\mathrm{Rb} / \mathrm{Ba}$ and $\mathrm{K} / \mathrm{Ba}$ ratios in leucogranites (Fig. 11) suggest that any such mechanism likely involved feldspar fractionation. However, the chemically least evolved leucogranite samples have $\mathrm{Eu} / \mathrm{Eu}^{*}$ ratios that exceed the average value for Yfqj, arguing against a parental role for the latter. A possible relationship involving fractionation of ferromagnesian silicate minerals also is precluded by similar $\mathrm{FeOt} /(\mathrm{FeOt}+\mathrm{MgO})$ ratios of Yfaj and associated leucogranites (Fig. 9G). In addition, the relative abundance of leucogranites is inconsistent with an origin involving differentiation of low-silica charnockite. Finally, the widespread biotite monzogranite (Ybg), which is compositionally transitional between penecontemporaneous low-silica charnockite (Yfqj) and associated leucogranites ( $\mathrm{Ygr}$ and $\mathrm{Ygg}$ ), is resolvably younger (circa $1030 \mathrm{Ma})$ than the latter (1050-1063 Ma). As a result, the range in compositional data characterizing plutons of Magmatic Interval III is unlikely to represent a sequence in which low-silica charnockite, biotite-rich monzogranite, and leucogranites represent successive stages of differentiation.

\subsection{Source characteristics and production of parental magmas}

Most Blue Ridge Mesoproterozoic rocks display compositions that are transitional between I- and A-type granitoids (Fig. 13); however, geochemical features of the low-silica charnockite (Yfqj) and biotite monzogranite (Ybg) of Magmatic Interval III are more indicative 

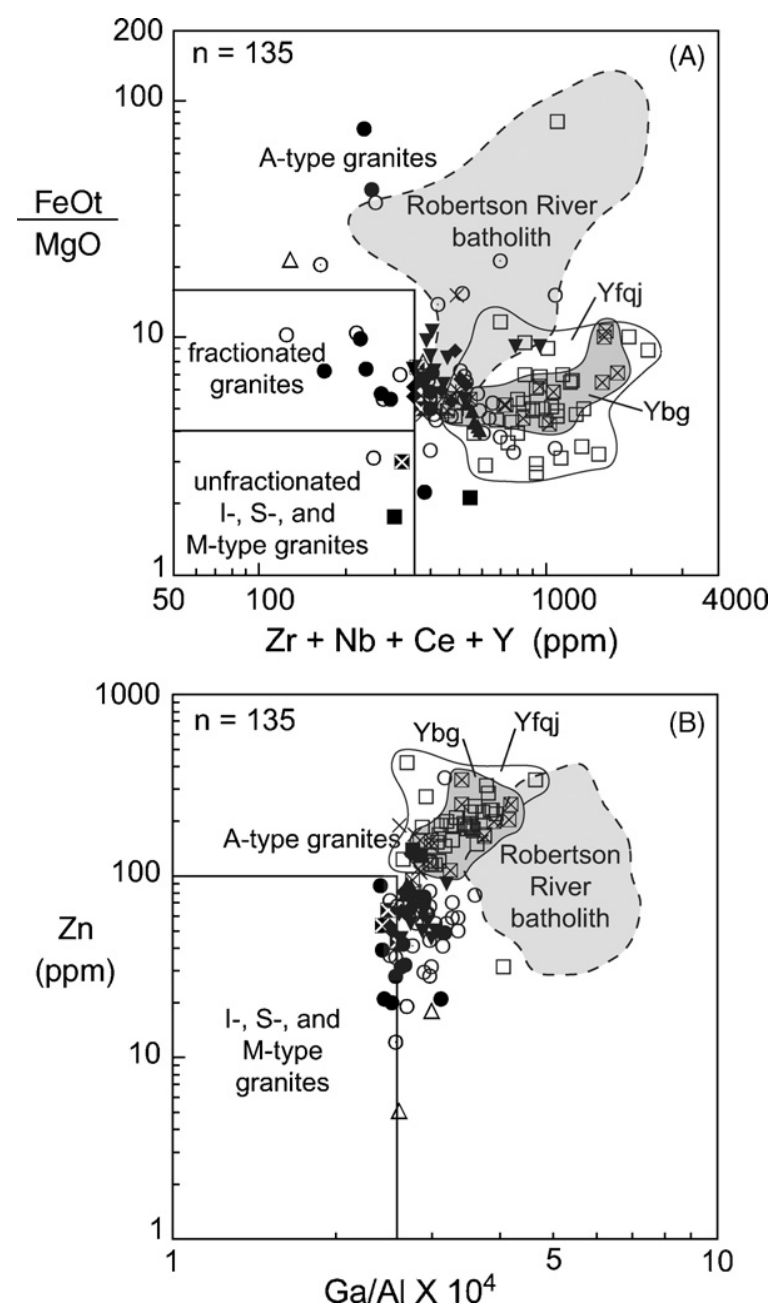

Fig. 13. Plots of $\mathrm{Zr}+\mathrm{Nb}+\mathrm{Ce}+\mathrm{Y}$ vs. $\mathrm{FeOt} / \mathrm{MgO}(\mathrm{A})$ and $\mathrm{Ga} / \mathrm{Al} \times 10^{4}$ vs. $\mathrm{Zn}$ (B) for rocks of the study area. Diagrams modified after Whalen et al. (1987). Symbols are the same as in Fig. 4. Data fields are outlined for biotite monzogranite (Ybg) and low-silica charnockite (Yfqj) emplaced during Magmatic Interval III. Dashed and shaded field encloses data (34 analyses) for metaluminous A-type granitoids of the Neoproterozoic Robertson River batholith from Virginia (Tollo and Aleinikoff, 1996).

of A-type affinity. Similar variation is characteristic of other Mesoproterozoic suites in eastern North America, including examples from the Central Gneiss Belt of the Canadian Grenville Province (Slagstad et al., 2004), Adirondack Highlands (McLelland and Whitney, 1990), and New Jersey Highlands (Volkert, 2004). Like the Blue Ridge rocks, these suites span a considerable age range and were produced in an evolving tectonic framework. HFSE concentrations of most Blue Ridge rocks are comparable, at lower $\mathrm{FeOt} / \mathrm{MgO}$ and $\mathrm{Ga} / \mathrm{Al}$ ratios, to values characteristic of metaluminous A-type granitoids from the extension-related circa 735-700 Ma Robertson River

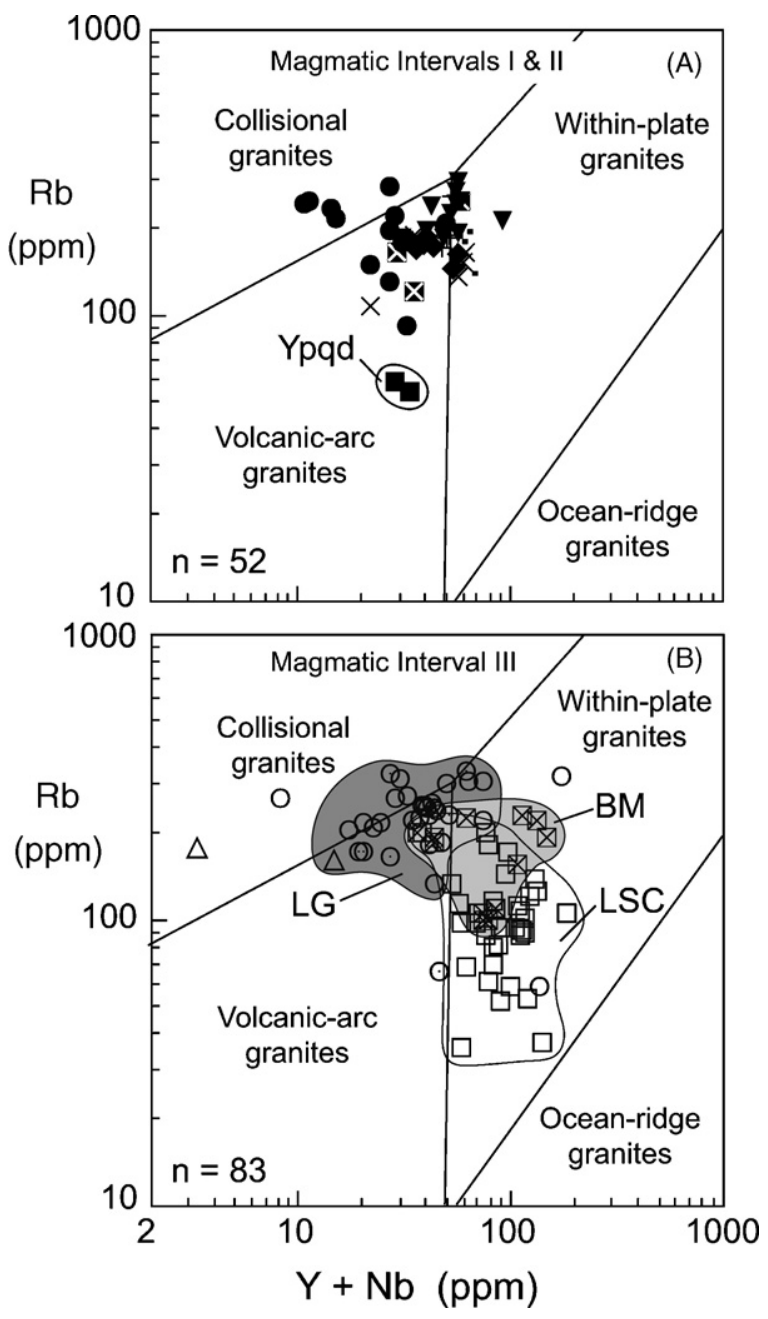

Fig. 14. Plots of $(Y+N b)$ vs. $\mathrm{Rb}$ for rocks emplaced during Magmatic Intervals I and II (A) and III (B). Diagrams modified after Pearce et al. (1984). All data are expressed in parts per million. Symbols are the same as in Fig. 4. Data fields are outlined for leucogranites (LG, units Ylgg, Ygg, and Ygr), biotite monzogranite (BM; unit Ybg) and low-silica charnockite (LSC, unit Yfaj) emplaced during Magmatic Interval III.

batholith that intruded the Blue Ridge core east of the study area (Fig. 2) (Tollo and Aleinikoff, 1996). However, only the low-silica charnockite (Yfaj) and biotite monzogranite (Ybg) are also characterized by moderately high $\mathrm{Ga} / \mathrm{Al}$ ratios and elevated concentrations of $\mathrm{Y}+\mathrm{Nb}$ and $\mathrm{Zn}$ (Figs. 13B and 14), compositional features typical of A-types (Whalen et al. (1987). Such geochemical variation likely results from a combination of differentiation (Collins et al., 1982) and source-related characteristics (Eby, 1992).

Compositionally transitional Blue Ridge rocks plot in an equivocal region of the tectonic discrimination diagrams of Pearce et al. (1984) (Fig. 14). Data for nearly all 
rocks of Magmatic Intervals I and II plot near the triplepoint intersection defined by compositional fields of collisional, volcanic-arc, and within-plate granites; however, samples of the circa 1160 Ma low-silica charnockite (Ypqd) plot solely in the volcanic-arc field. Similar to most older rocks, leucogranites of Magmatic Interval III plot near the triple-point intersection; however, less evolved biotite monzogranite (Ybg) and low-silica charnockite (Yfqj) exhibit increasing HFSE enrichment and $\mathrm{Rb}$ depletion. Förster et al. (1997) concluded that tectonic discrimination diagrams such as the $\mathrm{Nb}+\mathrm{Y}$ versus $\mathrm{Rb}$ plot (Fig. 14), while useful in many cases for identifying the tectonic environment of granitoid emplacement, are more likely to reflect compositional characteristics of sources involved in magma generation. Geochemical characterstics of granitoids of Magmatic Intervals I and II, and the leucogranites emplaced during Magmatic Interval III, correspond closely to those of syn- to post-collisional granitoids (Förster et al., 1997). This chemical signature is a result of the mixed sources involved in magma generation within recently developed (or still developing) orogens, where both continental crust and arc-related materials are commonly present (Förster et al., 1997). Data for the circa 1050 Ma lowsilica charnockite (Yfqj) plot well inside the within-plate granite field (Fig. 14), distinct from nearly all other Blue Ridge rocks. Such compositions are especially characteristic of post-collisional granitoids emplaced within extensional, encratonic environments (Förster et al., 1997). A post-collisional setting is consistent with field and geochronologic characteristics of this undeformed charnockite that intruded Blue Ridge crust about 30 m.y. after initiation of widespread orogenic activity (Tollo et al., 2004a). Sources for such A-type magmas range from lower crustal to mantle-derived but petrogenesis appears in most cases to involve a mantle contribution through either direct (e.g. source of primary magmas) or indirect (e.g. source of heat responsible for melting) processes (Turner et al., 1992a; Nédélec et al., 1995; Landenberger and Collins, 1996; Frost et al., 1999). Geochemical data for $1028 \pm 9$ Ma biotite monzogranite (Ybg) are transitional between the penecontemporaneous leucogranites and low-silica charnockite.

Association of fractionated I-type and A-type granites is not unusual worldwide (e.g. Landenberger and Collins, 1996). Although A-types are characterized by lower $\mathrm{Eu} / \mathrm{Eu}^{*}$, higher $\mathrm{Ga} / \mathrm{Al}$, and higher Th, features typically indicative of igneous differentiation, Eby (1992) did not observe a continuum of compositions and proposed that A-types are not derived from I-types through fractionation. This conclusion is supported by the Blue Ridge example where A-type charnockite (Yfqj) is less chemically evolved than associated transitional granitoids. Lack of petrologic relationship between transitional Blue Ridge granitoids and A-type charnockite could therefore result from differences in sources. The influence of sources on the geochemistry of A-types and fractionated I-types has been the subject of numerous multidisciplinary studies including various combinations of experimental, geochemical, and isotopic investigations. Models concerning generation of A-types and associated transitional granitoids involve the following mechanisms: (1) partial melting of, or fractionation from, a mafic source derived directly or indirectly from the mantle (Frost et al., 2001; Nédélec et al., 1995; Turner et al., 1992a), (2) partial melting of a residual crustal source (Landenberger and Collins, 1996; Whalen et al., 1987; Clemens et al., 1986), and (3) partial melting of an intermediate crustal source (Menuge et al., 2002; PatiñoDouce, 1997; Creaser et al., 1991).

Frost and Frost (1997) proposed that some metaluminous A-type granitoids characterized by high $\mathrm{K}_{2} \mathrm{O}$ contents and $\mathrm{FeOt} /(\mathrm{FeOt}+\mathrm{MgO})$ ratios are produced through either extreme differentiation of mantle-derived tholeiitic basalts or through partial melting of underplated basalts and their differentiates. The presence of ilmenite is a critical feature because it indicates that crystallization proceeded under relatively reducing redox conditions (Ishihara, 1977). Because some tholeiitederived suites include chemically unevolved rocks (e.g. Frost et al., 1999; Anderson et al., 2003), this model is an attractive option to explain the origin of the $\mathrm{Fe}$ rich, low-silica charnockite (Yfaj) of Magmatic Interval III. However, this pluton likely crystallized at relatively high $f\left(\mathrm{O}_{2}\right)$ because it contains abundant primary magnetite. Moreover, $\varepsilon_{\mathrm{Nd}}$ values for multiple samples of Yfqj (Table 5) range from 0.0 to +0.3 , indicating that this charnockite is unlikely to be derived from isotopically juvenile sources. Likewise, $\varepsilon_{\mathrm{Nd}}$ values of 0.7 to +1.2 (Table 5) rule out an origin involving juvenile sources for all of the other more evolved, ilmenite-bearing, transitional granitoids.

The dominance of relatively silica-rich Mesoproterozoic rocks produced repeatedly throughout a $\sim 150$ m.y. period in the Blue Ridge suggests that these rocks were derived from crustal sources. Emslie et al. (1994) proposed a similar origin for granitoids of the Nain AMCG Plutonic Suite in Labrador. Compositions of the least chemically evolved siliceous rocks indicate that parental magmas must have had $\mathrm{SiO}_{2}$ contents $<65 \%$ and were probably mildly metaluminous (Figs. 9 and 10). Landenberger and Collins (1996) considered crustal sources in evaluating possible relationships between older I-type and younger A-type granitoids of Trias- 
sic age that are closely associated in eastern Australia, proposing that water-undersaturated partial melting of K-rich, mafic to intermediate lower crust produced magmas that were parental to both types. An important aspect of this model is that an earlier melting episode that gave rise to parental magmas for the I-types resulted in dehydration of the residue so that subsequent melting produced water-undersaturated A-type magmas. However, Cullers et al. (1993) noted that such melting will deplete or significantly reduce alkali feldspar and quartz in the source, making it unlikely that such sources would generate parental magmas suitable to produce the chemically evolved granitoids of the Blue Ridge. In addition, Patiño-Douce (1997) noted that partial melting of such residues cannot produce granitic liquids with the high $\mathrm{TiO}_{2} / \mathrm{MgO}$ ratios that are typical of A-type granitoids in general and which are characteristic of virtually all samples analyzed in this study (Table 4).

Patiño-Douce (1997) and Skjerlie and Johnston (1993) demonstrated that granitoids similar in composition to the Blue Ridge rocks could be derived by partial melting of calc-alkaline sources and that previous production of I-type magma was not necessary to develop A-type features. Patiño-Douce (1997) performed melting experiments on tonalite and granodiorite at $950{ }^{\circ} \mathrm{C}$ and both 4 and $8 \mathrm{kbar}$. Results from the tonalite indicate that mildly metaluminous melt produced at $4 \mathrm{kbar}$, and weakly peraluminous melt formed at $8 \mathrm{kbar}$, are similar in composition to the silicic granitoids of this study, especially the leucogranites (Fig. 10). Moreover, PatiñoDouce (1997) demonstrated the importance of reactions involving plagioclase and orthopyroxene in developing compositional characteristics similar to the Blue Ridge silicic granitoids, including high $\mathrm{Fe} / \mathrm{Mg}$ and $\mathrm{TiO}_{2} / \mathrm{MgO}$, moderately elevated $\mathrm{Ga} / \mathrm{Al}$, depletion in $\mathrm{Sr}$ and $\mathrm{Eu}$, and enrichment in HFSE. We propose that compositional similarity between Blue Ridge granitoids and run products of Patiño-Douce (1997) constitutes strong evidence that comparable calc-alkaline lithologies served as an important component of sources that produced parental magmas for the more siliceous rocks of this study. However, such experimental results offer only approximations of the mixed, compositionally complex sources that likely exist at depth within long-lived orogenic zones formed by progressive amalgamation and tectonic juxtaposition of heterogeneous lithospheric components. Parental magmas for Blue Ridge rocks likely were somewhat less siliceous than the experimental products, and therefore were derived either through a greater degree of melting of a tonalitic source or involved lithologically complex tonalitic sources that also included a more mafic component. Menuge et al. (2002) likewise proposed that
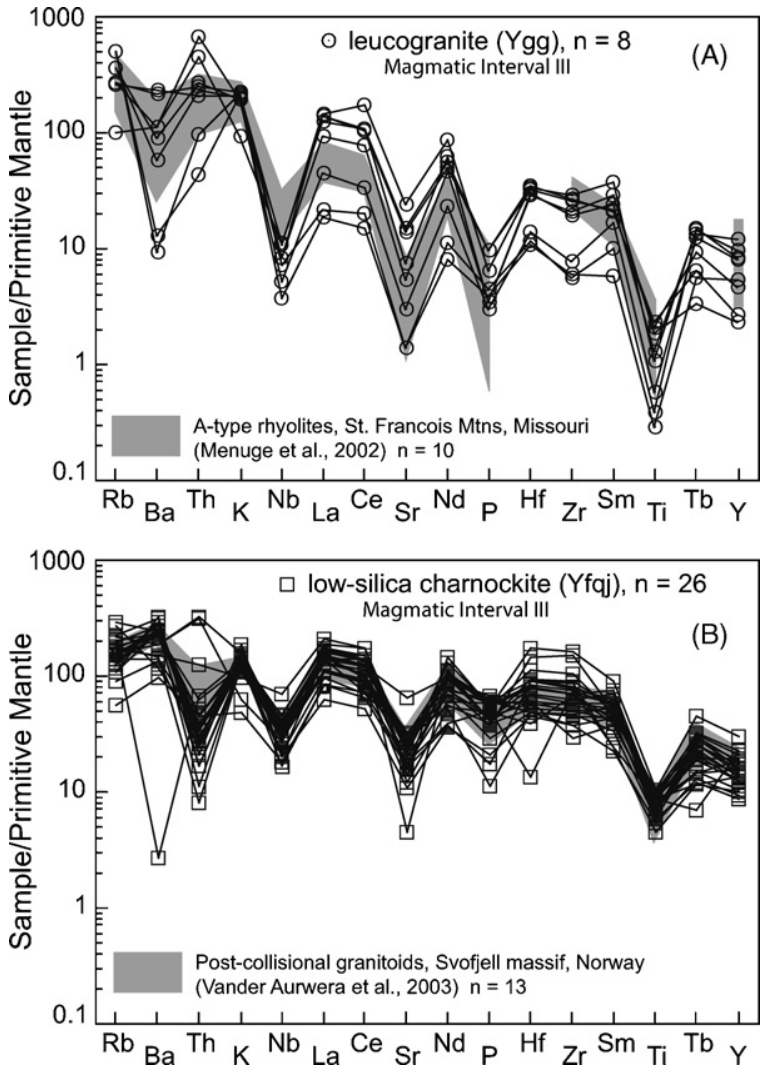

Fig. 15. Primitive mantle-normalized elemental variation diagram comparing the composition of a representative Blue Ridge leucogranite (Ygg) of Magmatic Interval III to A-type rhyolites from the St. Francois Mountains in Missouri (Menuge et al., 2002) (A) and low-silica charnockite (Yfqj) of Magmatic Interval III to A-type granitoids from the Svöfjell massif in southern Norway (Vander Auwera et al., 2003) (B).

1.45-1.60 Ga A-type rhyolites of the St. Francois Mountains in Missouri, which are compositionally similar to leucogranites of this study (Fig. 15A), were derived from dominantly calc-alkaline source materials by processes that likely involved large degrees of melting. Compositional affinities between the Blue Ridge and St. Francois rocks include similar fractionation-related depletions in $\mathrm{Ba}$ (feldspar), P (apatite), and Ti (ilmenite), as well as probable source-related depletion in $\mathrm{Nb}$ (Fig. 15A). Similarly, Daly and McLelland (1991) determined that melting of largely calc-alkaline sources was responsible for production of some AMCG and Ottawan magmatism in the Adirondack Highlands. Collectively, these rocks are compositionally similar and temporally comparable to the Blue Ridge granitoids.

Melts produced in the Patiño-Douce (1997) study do not replicate the average composition of low-silica charnockite (Yfqj) and biotite monzogranite (Ybg) of Magmatic Interval III, suggesting that similar tonalitic 
sources are unsuitable precursors to parental magmas for these rocks. These relatively unevolved plutons have low to moderate silica concentrations (Table 4) and $\varepsilon_{\mathrm{Nd}}$ values that are similar to those of the more silicarich granitoids and leucogranites (Table 5), suggesting derivation by melting of chemically less evolved crustal sources of comparable age. Vander Auwera et al. (2003) described post-collisional biotite + hornblende-bearing granitoids from the Svöfjell massif in southern Norway that correspond closely to the $\sim 1050 \mathrm{Ma}$ low-silica Atype charnockite of this study (Fig. 15B). They proposed that gabbroic parental magmas were derived by partial melting of relatively undepleted mafic sources that were both hydrated and potassic. We envision similar sources for the low-silica charnockite which, like the Svöfjell granitoids, contains primary amphibole and a relatively oxidized mineral assemblage. Such lower crustal sources likely melted in response to increased temperatures following the onset of major deformation at about $1080 \mathrm{Ma}$.

Zircons in Magmatic Interval III granitoids support separate origins for granitic and jotunitic members of the Blue Ridge suite. Lack of inherited zircon in the low-silica charnockite is a likely result of the high temperatures and high solubility of $\mathrm{Zr}$ in such melts, characteristics that would result in enhanced dissolution of entrained zircon (Watson and Harrison, 1983). Less likely, lack of inherited component could indicate absence of zircon in the source. In either case, the common presence of inherited zircon in the non-leucocratic granitoids and leucogranites emphasizes their probable derivation from sources different from those of the charnockite, and suggests that the former are unlikely differentiation products of the latter. Zircons in biotite monzogranite (Ybg) contain evidence of inherited component, suggesting a petrologic lineage similar to the granitic rocks. Compositional and mineralogical characteristics of this chemically less evolved, more hydrous granitoid suggest that it formed from crustal sources that were more mafic than those that produced the associated granitoids.

The geodynamic model of Turner et al. (1992b) suggests that increased temperatures responsible for production of parental magmas for the post-tectonic charnockite (Yfqj) may have resulted from thinning of the lithospheric mantle. Removal of part or all of the lithospheric mantle could have been achieved by either delamination or convective thinning but, in either case, would likely have been accompanied by increased temperatures and intrusion of potassic magmas (Turner et al., 1996). Emplacement of the low-silica charnockite may thus mark the onset of a new melting regime in the underlying source regions. As a consequence, Magmatic Interval
III includes plutons derived from at least two types of sources: (1) tonalitic crustal sources that produced nonleucocratic granitoids and leucogranites episodically in response to crustal thickening over $\sim 150$ m.y., and (2) potassic mafic sources that melted only in the postkinematic environment in response to increased thermal input from the asthenosphere. High temperatures that accompanied production of mafic parental magmas probably resulted in increased solubility of accessory phases that, in turn, led to higher concentrations of HFSE and enhanced A-type affinity of the low-silica charnockite (Collins et al., 1982).

The $\sim 1161$ Ma low-silica charnockite (Ypqd), characterized by magnesian compositions (Fig. 9D and E), low HFSE concentrations (Fig. 11), possible volcanicarc affinity (Fig. 14A), and the least evolved $\varepsilon_{\mathrm{Nd}}$ values of the Blue Ridge suite (Fig. 12), probably was not produced from sources comparable to those that gave rise to other Blue Ridge rocks. The unique compositional characteristics of this body raise the possibility that it formed from different crustal sources, perhaps involving greater volcanic-arc component.

\section{Tectonic significance}

The three episodes of Blue Ridge magmatic activity span over 150 m.y. (Fig. 16; Table 3) of tectonic evolution. A major period of deformation, probably correlative to the regional Ottawan orogeny (McLelland et al., 2001; Rivers, 1997), occurred at 1078-1050 Ma. A similar time frame is recorded by largely granitic rocks of the Adirondacks where Ottawan deformation and metamorphism occurred at 1090-1045 Ma (McLelland et al., 2001) (Fig. 16).

The timing of episodic Blue Ridge igneous activity corresponds to periods of magmatism in the Adirondack Highlands where dominantly granitic plutonism occurred at 1175-1140, 1103-1093, and 1060-1040 Ma (Fig. 16) (McLelland et al., 1996, 2001). This correspondence in magmatic timing, together with temporal overlap of Ottawan orogenesis, provides strong evidence for the widespread nature of Grenvillian processes. The recurrence of magmatism in the Blue Ridge implies the existence of episodic thermal flux. Significantly, Magmatic Interval I ended about $60 \mathrm{~m}$.y. prior to major deformation and, presumably, crustal thickening in the Blue Ridge. As a result, this magmatism does not appear to be directly related to Ottawan orogenesis. McLelland et al. (2004) proposed that Adirondack AMCG magmatism, which shares many compositional features with Magmatic Interval I granitoids including tholeiitic affinity and A-type characteristics (McLelland and Whitney, 


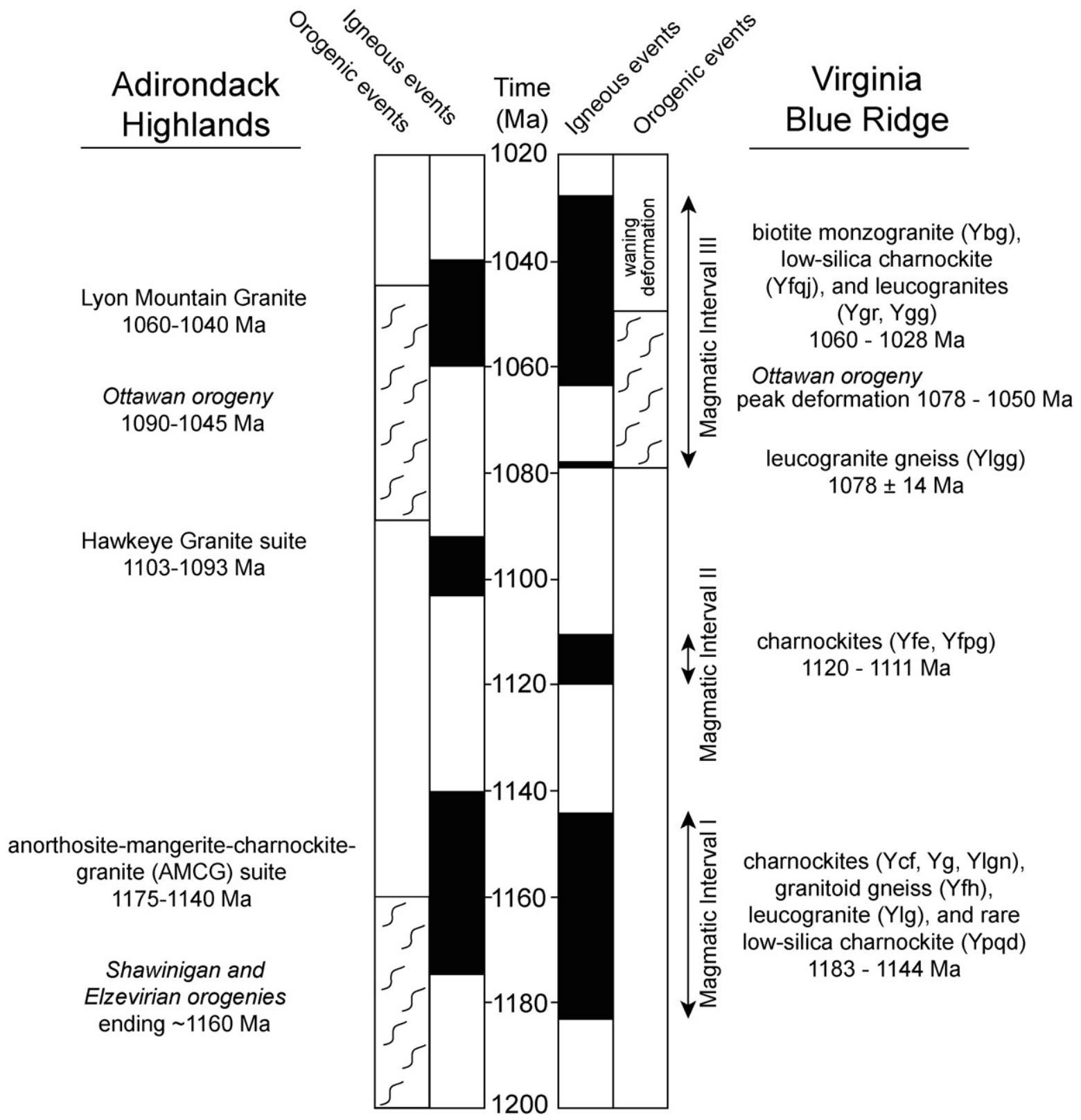

Igneous activity

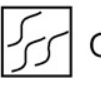

Orogenic activity (names in italics)

Fig. 16. Comparison of igneous and orogenic histories of the northern Virginia Blue Ridge (this study) and the Adirondack Highlands. Igneous crystallization ages for the latter are based on zircon U-Pb data from McLelland et al. (1996, 2001, 2004).

1990), occurred in response to lithospheric delamination following the 1200-1150 Ma Shawinigan orogeny, and it is therefore possible that some or all of the Magmatic Interval I plutonism in the Blue Ridge could have a similar origin involving an older orogenic episode. However, because there is presently no evidence for
Shawinigan orogenic activity in the Virginia Blue Ridge, the relevance of the Adirondack model is difficult to assess. The occurrence of mafic, $\sim 1160 \mathrm{Ma}$ low-silica charnockite (Ypqd) indicates that the Magmatic Interval I sequence is, like the Adirondack AMCG interval, compositionally bimodal but dominated by granitoids. The 
presence of such mafic rocks points to the existence of high-temperature magmas that might have served as heat sources responsible for crustal melting that ultimately produced contemporaneous granitoids.

Charnockites of Magmatic Interval II are compositionally indistinguishable from similar rocks of Magmatic Interval I and were therefore likely derived from similar calc-alkaline sources. McLelland et al. (1996) noted that emplacement of the 1100-1090 Ma Hawkeye Granite suite in the Adirondacks coincided with magmatism in the Mid-Continent rift (MCR) (Van Schmus et al., 1993) and suggested that Adirondack plutonism was related to MCR activity. Magmatic Interval II, which may also include leucogranites of $1112 \mathrm{Ma}$ age (Aleinikoff et al., 2000), occurred immediately prior to initiation of major magmatism in the MCR. However, it is unlikely to be related to MCR activity because the latter is linked to mantle plume activity for which evidence is lacking within the Grenville orogen. Considering compositional similarities with older Blue Ridge granitoids, we view the Magmatic Interval II activity as indicating reinvigoration of Magmatic Interval I processes following an apparent 24 m.y. hiatus.

The age range of Magmatic Interval III plutons overlaps that of the Adirondack Lyon Mountain Granite suite (Fig. 16) and, like the latter, the Blue Ridge rocks are lateto post-tectonic. However, whereas the Lyon Mountain suite is dominantly felsic and derived from similar crustal sources (McLelland et al., 1996), the Blue Ridge plutons include significant volumes of low-silica rocks that were derived from more primitive sources. The apparent compositional continuity of the Blue Ridge suite is produced by penecontemporaneous plutons including relatively unevolved rocks derived from mafic sources and more chemically evolved counterparts derived from intermediate sources. In this way, insight provided by geochronologic data, demonstrating that the evolved leucogranites are considerably older than compositionally transitional rocks, provides a critical constraint on possible petrogenetic models.

The interval $1200-1000 \mathrm{Ma}$ is likewise marked by abundant magmatism in the Grenville province of Canada (Rivers, 1997). This plutonism, which occurs along the full length of the province but is concentrated in the southeastern sector, includes mafic rocks, AMCG complexes of varying size, and granitoid bodies that are not associated with AMCG rocks. Most of this synto post-tectonic plutonic activity bears "within-plate" compositional characteristics similar to the majority of Blue Ridge rocks described in this study, and likely resulted from similar delamination-related processes that accompanied and followed two major orogenic episodes
(Corrigan and Hanmer, 1995; Rivers, 1997). As a result, these rocks collectively signify a tectonically important transition from pre-1190 Ma, dominantly calc-alkaline magmatism related to arc convergence and subduction. The compositional and temporal similarities between the oldest Blue Ridge rocks (i.e. Magmatic Interval I and, possibly, II) and those of the Adirondacks and Canadian Grenville province may foreshadow future recognition of a pre-Ottawan orogenic event in the basement terrane of northern Virginia.

\section{Conclusions}

The observations and data of this study support the following conclusions regarding the Grenvillian geology of the northern Virginia Blue Ridge.

1. Grenvillian intrusive activity produced plutons composed of: (1) low-silica charnockite, (2) leucogranite, (3) non-leucocratic granitoid (including charnockite), and (4) intermediate granitoid. Crystallization ages define three periods of plutonic activity occurring at 1183-1144 Ma (Magmatic Interval I), 1120-1111 Ma (Magmatic Interval II), and 1078-1028 Ma (Magmatic Interval III). High-temperature, ductile deformation occurred at 1078-1050 Ma and likely corresponds to the regional Ottawan orogeny. The timing of episodic igneous activity and orogenesis corresponds to similar periods in the Adirondack Highlands.

2. Chemically evolved non-leucocratic granitoids (Magmatic Intervals I and II) and leucogranites (Magmatic Intervals I and III) are compositionally transitional between I- and A-type granitoids. Comparison with experimental data suggests that parental magmas were produced either by partial melting of mixed mafic and tonalitic sources or by relatively high degrees of melting of dominantly tonalitic sources.

3. The circa $1050 \mathrm{Ma}$ low-silica charnockite (Yfqj) was emplaced following peak deformation and is chemically distinctive in displaying A-type characteristics. These magnetite-bearing rocks were likely derived from relatively oxidized parental magmas produced by partial melting of potassic, dominantly mafic, lower crustal sources. In contrast, associated intermediate biotite monzogranite was probably derived from sources similar to those of the more silicic granitoids.

4. Linear major-element compositional trends defined by plutons of Magmatic Interval III involve magmas that were emplaced episodically over $\sim 30$ m.y. and 
which were derived from different crustal sources. These apparent trends do not result from differentiation and thus underscore the importance of applying precise geochronology for interpretation of geochemical characteristics of ancient igneous terranes.

5. Differences in geochemical composition and apparent tectonic affinity of Blue Ridge Grenvillian rocks reflect source characteristics, modified by differentiation. In long-lived orogenic regions composed at depth of heterogeneous source materials, such rocks may define continuous compositional spectra, making distinction between source-related I- and A-types difficult. Without precise geochronologic data, geochemical data are of limited value in developing an enhanced understanding of the tectonic significance of local magmatism.

\section{Acknowledgements}

This research was supported in part by grants from the Educational Geologic Mapping Program administered by the U.S. Geological Survey. Reviews by T. Slagstad, J. McLelland, R.A. Ayuso, M.J. Bartholomew, and S. Southworth are gratefully acknowledged. Neutron activation analyses at Oregon State University were supported by the Reactor Sharing Program administered through the U.S. Department of Energy. J.M. Rhodes, P. Dawson, and M. Vollinger of the Ronald B. Gilmore Analytical Geochemistry Laboratory located at the University of Massachusetts in Amherst provided valuable assistance with the XRF analyses. Assistance with mineral separation by E. Yacob, help with SEM imaging by I. Brownfield (both USGS), and the diligent efforts of research assistants A. Antignano, A. Ascrizzi, C. Claflin, L. Olsen, E. Pogue, S. (Nyman) Green, and B. Sabelli from George Washington University contributed significantly to the project. Their efforts are greatly appreciated. Our gratitude is extended to $\mathrm{S}$. Southworth of the USGS who provided logistical support.

\section{References}

Aleinikoff, J.N., Burton, W.C., Lyttle, P.T., Nelson, A.E., Southworth, C.S., 2000. U-Pb geochronology of zircon and monazite from Mesoproterozoic granitic gneisses of the northern Blue Ridge, Virginia and Maryland, USA. Precambrian Res. 99, 113-146.

Aleinikoff, J.N., Schenck, W.S., Plank, M.O., Srogi, L., Fanning, C.M., Kamo, S.L., Bosbyshell, H., 2006. Deciphering igneous and metamorphic events in high grade rocks of the Wilmington Complex, Delaware: morphology, CL and BSE zoning, and SHRIMP U-Pb geochronology of zircon and monazite. Geol. Soc. Am. Bull. 118, $39-64$.

Aleinikoff, J.N., Zartman, R.E., Walter, M., Rankin, D.W., Lyttle, P.T., Burton, W.C., 1995. U-Pb ages of metarhyolites of the Catoctin and Mount Rogers Formations, central and southern Appalachians: evidence for two pulses of Iapetan rifting. Am. J. Sci. 295, 428-454.

Anderson, I.C., Frost, C.D., Frost, B.R., 2003. Petrogenesis of the Red Mountain pluton, Laramie anorthosite complex, Wyoming: implications for the origin of A-type granite. Precambrian Res. 124, 243-267.

Bailey, C.M., Simpson, C., 1993. Extensional and contractional deformation in the Blue Ridge province, Virginia. Geol. Soc. Am. Bull. $105,411-422$.

Bailey, C.M., Berquist, P.J., Mager, S.M., Knight, B.D., Shotwell, N.L., Gilmer, A.K., 2003. Bedrock Geology of the Madison Quadrangle, Virginia. Virginia Division of Mineral Resources Publication 157.

Bailey, C.M., Simpson, C., De Paor, D.G., 1994. Volume loss and tectonic flattening in granitic mylonites from the Blue Ridge province, central Appalachians. J. Struct. Geol. 16, 1403-1416.

Bartholomew, M.J., 1977. Geology of the Greenfield and Sherando Quadrangles, Virginia. Virginia Division of Mineral Resources Publication 4, 43 pp.

Bartholomew, M.J., Gathright II, T.M., Henika, W.S., 1981. A tectonic model for the Blue Ridge in central Virginia. Am. J. Sci. 281, 1164-1183.

Bartholomew, M.J., Lewis, S.E., 1984. Evolution of Grenville massifs in the Blue Ridge geologic province, southern and central Appalachians. In: Bartholomew, M.J., Force, E.R., Sinha, A.K., Herz, N. (Eds.),Evolution of Grenville massifs in the Blue Ridge geologic province, southern and central Appalachians. The Grenville Event in the Appalachians and Related Topics. Geological Society of America Special Paper 194, pp. 229-254.

Black, L.P., Kamo, S.L., Allen, C.M., Davis, D.W., Aleinikoff, J.N., Valley, J.W., Mundil, R., Campbell, I.H., Korsuch, R.J., Williams, I.S., Foudoulis, C., 2004. Improved ${ }^{206} \mathrm{~Pb} /{ }^{238} \mathrm{U}$ microprobe geochronology by the monitoring of a trace-element-related matrix effect; SHRIMP, ID-TIMS, ELA-ICP-MS and oxygen isotope documentation for a series of zircon standards. Chem. Geol. 205, 115-140.

Bloomer, R.O., Werner, H.J., 1955. Geology of the Blue Ridge in central Virginia. Geol. Soc. Am. Bull. 66, 579-606.

Burton, W.C., Southworth, C.S., 2004. Tectonic evolution of the northern Blue Ridge massif, Virginia and Maryland.Tectonic evolution of the northern Blue Ridge massif, Virginia and Maryland. In: Tollo, R.P., Corriveau, L., McLelland, J., Bartholomew, M.J. (Eds.), Proterozoic Tectonic Evolution of the Grenville Orogen in North America. Geological Society of America Memoir No. 197, pp. 477-493.

Burton, W.C., Froelich, A.J., Pomeroy, J.S., Lee, K.Y., 1995. Geology of the Waterford quadrangle, Virginia and Maryland, and the Virginia part of the Point of Rocks quadrangle. US Geol. Surv. Bull. 2095, 30 (Scale 1:24,000).

Clemens, J.D., Holloway, J.R., White, A.J.R., 1986. Origin of an A-type granite: experimental constraints. Am. Mineral. 71, 317324.

Collins, W.J., Beams, S.D., White, A.J.R., Chappell, B.W., 1982. Nature and origin of A-type granites with particular reference to southeastern Australia. Contrib. Mineral. Petrol. 80, 189-200.

Corrigan, D., Hanmer, S., 1995. Anorthosites and related granitoids in the Grenville orogen: a product of convective thinning of the lithosphere? Geology 25, 61-64.

Creaser, R.A., Price, R.C., Wormald, R.J., 1991. A-type granites revisited: assessment of a residual source model. Geology 19, 163-166.

Cullers, R.L., Stone, J., Anderson, J.L., Sassarini, N., Bickford, M.E., 1993. Petrogenesis of Mesoproterozoic Oak Creek and West McCoy Gulch plutons, Colorado: an example of cumulate unmix- 
ing of mid-crustal, two-mica granite of orogenic affinity. Precambrian Res. 62, 139-169.

Daly, J.S., McLelland, J.M., 1991. Juvenile Middle Proterozoic crust in the Adirondack Highlands, Grenville province, northeastern North America. Geology 19, 119-122.

Dalziel, I.W.D., 1997. Overview: Neoproterozic-Paleozoic geography and tectonics: review, hypothesis, environmental speculations. Geol. Soc. Am. Bull. 109, 16-42.

DePaolo, D.J., 1981. Neodymium isotopes in the Colorado Front Range and crust-mantle evolution in the Proterozoic. Nature 291, 193-196.

Eby, G.N., 1992. Chemical subdivision of the A-type granitoids: petrogenetic and tectonic implications. Geology 20, 641-644.

Emslie, R.F., 1978. Anorthosite massifs, rapakivi granites and late Proterozoic rifting of North America. Precambrian Res. 7, 61-98.

Emslie, R.F., Hamilton, M.A., Thériault, R.J., 1994. Petrogensis of a Mid-Proterorzoic Anorthosite-Mangerite-Charnockite-Granite (AMCG) Complex: isotopic and chemical evidence from the Nain Plutonic Suite. J. Geol. 102, 539-558.

Evans, N., 1991. Latest Precambrian to Ordovician metamorphism in the Virginia Blue Ridge: origin of the contrasting Lovingston and Pedlar basement massifs. Am. J. Sci. 291, 425-452.

Förster, H.-J., Tischendorf, G., Trumbull, R.B., 1997. An evaluation of the $\mathrm{Rb}$ vs. $(\mathrm{Y}+\mathrm{Nb})$ discrimination diagram to infer tectonic setting of silicic igneous rocks. Lithos 40, 261-293.

Frost, B.R., Frost, C.D., Hulsebosch, T.P., Swapp, S.M., 2000. Origin of the charnockites of the Louis Lake batholith, Wind River Range, Wyoming. J. Petrol. 41, 1759-1776.

Frost, C.D., Frost, B.R., 1997. Reduced rapakivi-type granites: the tholeiite connection. Geology 25, 647-650.

Frost, C.D., Frost, B.R., Chamberlain, K.R., Edwards, B.R., 1999. Petrogenesis of the $1.43 \mathrm{Ga}$ Sherman batholith, SE Wyoming, USA: a reduced rapakivi-type anorogenic granite. J. Petrol. 10, 17711802.

Frost, C.D., Bell, J.M., Frost, B.R., Chamberlain, K.R., 2001. Crustal growth by magmatic underplating: isotopic evidence from the northern Sherman batholith. Geology 29, 515-518.

Fullagar, P.D., Goldberg, S.A., Butler, J.R., 1997. Nd and Sr isotopic characterization of crystalline rocks from the southern Appalachian Piedmont and Blue Ridge, North and South Carolina.Nd and $\mathrm{Sr}$ isotopic characterization of crystalline rocks from the southern Appalachian Piedmont and Blue Ridge, North and South Carolina. In: Sinha, A.K., Whalen, J.B., Hogan, J.P. (Eds.), The Nature of Magmatism in the Appalachian Orogen. Geological Society of America Memoir No. 191, pp. 165-179.

Gathright II, T.M., 1976. Geology of the Shenandoah National Park in Virginia. Virginia Division of Mineral Resources Bulletin 86, 93 pp.

Gill, J.B., 1981. Orogenic Andesites and Plate Tectonics. SpringerVerlag, $401 \mathrm{pp}$.

Holmden, C., Dickin, A.P., 1995. Paleoproterozoic crustal history of the southwestern Grenville Province: evidence from $\mathrm{Nd}$ isotopic mapping. Can. J. Earth Sci. 32, 472-485.

Hughes, S.S., Lewis, S.E., Bartholomew, M.J., Sinha, A.K., Herz, N., 2004. Geology and geochemistry of granitic and charnockitic rocks in the central Lovingston massif of the Grenvillian Blue Ridge terrane.Geology and geochemistry of granitic and charnockitic rocks in the central Lovingston massif of the Grenvillian Blue Ridge terrane. In: Tollo, R.P., Corriveau, L., McLelland, J., Bartholomew, M.J. (Eds.), Proterozoic Tectonic Evolution of the Grenville Orogen in North America. Geological Society of America Memoir No. 197, pp. 549-569.
Ireland, T.R., Wooden, J.L., Persing, H.M., Ito, B., 1999. Geological applications and analytical development of the SHRIMP-RG. Eos. Trans. Am. Geophys. Union 80 (Suppl. 46), F1117.

Ishihara, S., 1977. The magnetite-series and ilmenite-series granitic rocks. Min. Geol. 27, 293-305.

Jonas, A.I. (Ed.), 1928. Geologic Map of Virginia. Virginia Division of Mineral Resources, Charlottesville, Virginia (Scale 1:500,000).

Karlstrom, K.E., Åhäll, K.-I., Harlan, S.S., Williams, M.L., McLelland, J., Geissman, J.W., 2001. Long-lived (1.8-1.0 Ga) convergent orogen in southern Laurentia, its extensions to Australia and Baltica, and implications for refining Rodinia. Precambrian Res. 111, 5-30.

Kilpatrick, J.A., Ellis, D.J., 1992. C-type magmas: igneous charnockites and their extrusive equivalents. In: Brown, P.E., Chappell, B.W. (Eds.),C-type magmas: igneous charnockites and their extrusive equivalents. The Second Hutton Symposium on the Origin of Granites and Related Rocks. Geological Society of America Special Paper 272, pp. 155-164.

Kretz, R., 1983. Symbols for rock-forming minerals. Am. Mineral. 68, 277-279.

Kunk, M.J., Burton, W.C., 1999. ${ }^{40} \mathrm{Ar} /{ }^{39} \mathrm{Ar}$ age-spectrum data for amphibole, muscovite, biotite, and K-feldspar samples from metamorphic rocks in the Blue Ridge anticlinorium, northern Virginia. U.S. Geological Survey Open-File Report OF-99-0552, 110 pp.

Landenberger, B., Collins, W.J., 1996. Derivation of A-type granites from dehydrated charnockitic lower crust: Evidence from the Chaelundi Complex, eastern Australia. J. Petrol. 37, 145-170.

Le Maitre, R.W., Bateman, P., Dudek, A., Keller, J., Lameyre, J., Le Bas, M.J., Sabine, P.A., Schmid, R., Sørensen, H., Streckeisen, A., Woolley, A.R., Zanettin, B., 1989. A Classification of Igneous Rocks and Glossary of Terms: Recommendations of the International Union of Geological Sciences Subcommision on the Systematics of Igneous Rocks. Blackwell Scientific Publications, 193 pp.

Ludwig, K.R., 1998. On the treatment of concordant uranium-lead ages. Geochim. Cosmochim. Acta 62, 665-676.

Ludwig, K.R., 2001. Squid, Version 1.02, A User's Manual. Berkeley Geochronology Center Special Publication 2, 21 pp.

Ludwig, K.R., 2003. Isoplot/Ex Version 3.00, A Geochronological Toolkit for Mircrosoft Excel. Berkeley Geochronology Center Special Publication 4, 73 pp.

Lukert, M.T., 1982. Uranium-lead isotope age of the Old Rag Granite, northern Virginia. Am. J. Sci. 282, 391-398.

Lukert, M.T., Nuckols, E.B., Clarke, J.W., 1977. Flint Hill Gneiss: a definition. Southeastern Geol. 19, 19-28.

Liogys, V.A., Tracy, R.J., 2004. Augite-inverted ferropigeonite thermometry in "charnockitic" rocks of the Grenvillian Blue Ridge, central Virginia. Geol. Soc. Am. Abstr. Programs 36 (2), 79.

McLelland, J., Whitney, P., 1990. Anorogenic, bimodal emplacement of anorthositic, charnockitic, and related rocks in the Adirondack Mountains, New York. In: Stein, H.J., Hannah, J.L. (Eds.), Anorogenic, bimodal emplacement of anorthositic, charnockitic, and related rocks in the Adirondack Mountains, New York. Ore-bearing Granite Systems: Petrogenesis and Mineralizing Processes. Geological Society of America Special Paper 246, pp. 301-315.

McLelland, J.M., Bickford, M.E., Hill, B.M., Clechenko, C.C., Valley, J.W., Hamilton, M.A., 2004. Direct dating of Adirondack massif anorthosite by U-Pb SHRIMP analysis of igneous zircon: implications for AMCG complexes. Geol. Soc. Am. Bull. 116, 1299-1317.

McLelland, J., Daly, J.S., McLelland, J.M., 1996. The Grenville Orogenic Cycle (ca. 1350-1000 Ma): an Adirondack perspective. Tectonophysics 265, 1-28. 
McLelland, J., Hamilton, M., Selleck, B., McLelland, J., Walker, D., Orrell, S., 2001. Zircon U-Pb geochronology of the Ottawan Orogeny, Adirondack Highlands, New York: regional and tectonic implications. Precambrian Res. 109, 39-72.

Menuge, J.F., Brewer, T.S., Seeger, C.M., 2002. Petrogenesis of metaluminous A-type rhyolites from the St. Francois Mountains, Missouri and the Mesoproterozoic evolution of the southern Laurentian margin. Precambrian Res. 113, 269-291.

Nédélec, A., Stephens, W.E., Fallick, A.E., 1995. The Panafrican Stratoid Granites of Madagascar: alkaline magmatism in a postcollisional extensional setting. J. Petrol. 36, 1367-1391.

Norrish, K., Chappell, B.E., 1967. X-ray fluorescence spectrography. In: Zussman, J. (Ed.),X-ray fluorescence spectrography. Physical Methods in Determinative Mineralogy. Academic Press, pp. 161-214.

Norrish, K., Hutton, J.T., 1969. An accurate X-ray spectrographic method for the analysis of a wide range of geologic materials. Geochem. Cosmochim. Acta 33, 431-454.

Owens, B.E., Samson, S.D., 2004. Nd isotopic constraints on the magmatic history of the Goochland terrane, easternmost Grenvillian crust in the southern Appalachians.Nd isotopic constraints on the magmatic history of the Goochland terrane, easternmost Grenvillian crust in the southern Appalachians. In: Tollo, R.P., Corriveau, L., McLelland, J., Bartholomew, M.J. (Eds.), Proterozoic Tectonic Evolution of the Grenville Orogen in North America. Geological Society of America Memoir No. 197, pp. 601-608.

Patiño-Douce, A.E., 1997. Generation of metaluminous A-type granites by low-pressure melting of calc-alkaline granitoids. Geology $25,743-746$.

Pearce, J.A., Harris, N.B.W., Tindle, A.G., 1984. Trace element discrimination diagrams for the tectonic interpretation of granitic rocks. J. Petrol. 25, 956-983.

Pettingill, H.S., Sinha, A.K., Tatsumoto, M., 1984. Age and origin of anorthosites, charnockites, and granulites in the Central Virginia Blue Ridge. Contrib. Mineral. Petrol. 85, 279-291.

Rämö, O.T., 1991. Petrogenesis of the Proterozoic rapakivi granites and related rocks of southeastern Fennoscandia: $\mathrm{Nd}$ and $\mathrm{Pb}$ isotopic and general chemical constraints. Geol. Soc. Finland Bull. 355, 161.

Rankin, D.W., Drake Jr., A.A., Glover III, L., Goldsmith, R., Hall, L.M., Murray, D.P., Ratcliffe, N.M., Read, J.F., Secor Jr., D.T., Stanley, R.S., 1989. Pre-orogenic terranes. In: Hatcher Jr., R.D., Thomas, W.A., Viele, G.W. (Eds.),Pre-orogenic terranes. The Appalachian-Ouachita orogen in the United States. The Geology of North America F-2, pp. 7-100.

Rankin, D.W., Drake Jr., A.A., Ratcliffe, N.M., 1990. Geologic map of the U.S. Appalachians showing the Laurentian margin and Taconic orogen. In: Hatcher Jr., R.D., Thomas, W.A., Viele, G.W. (Eds.), Geologic map of the U.S. Appalachians showing the Laurentian margin and Taconic orogen. The Appalachian-Ouachita Orogen in the United States. The Geology of North America F2, Plate 2.

Rivers, T., 1997. Lithotectonic elements of the Grenville Province: review and tectonic implications. Precambrian Res. 86, 117-154.

Rubatto, D., Williams, I.S., Buick, I.S., 2001. Zircon and monazite response to prograde metamorphism in the Reynolds Range, central Australia. Contrib. Mineral. Petrol. 140, 458-468.

Sinha, A.K., Bartholomew, M.J., 1984. Evolution of the Grenville terrane in the central Virginia Appalachians.Evolution of the Grenville terrane in the central Virginia Appalachians. In: Bartholomew, M.J., Force, E.R., Sinha, A.K., Herz, N. (Eds.), The Grenville Event in the Appalachians and Related Topics. Geological Society of America Special Paper 194, pp. 175-186.
Skjerlie, K.P., Johnston, A.D., 1993. Fluid-absent melting behavior of an F-rich tonalite gneiss at mid-crustal pressures: implications for the generation of anorogenic granites. J. Petrol 34, 785-815.

Slagstad, T., Culshaw, N.G., Jamieson, R.A., Ketchum, J.W.F., 2004. Early Mesoproterozoic tectonic history of the southwestern Grenville Province, Ontario: constraints from geochemistry and geochronology of high-grade gneisses.Early Mesoproterozoic tectonic history of the southwestern Grenville Province, Ontario: constraints from geochemistry and geochronology of high-grade gneisses. In: Tollo, R.P., Corriveau, L., McLelland, J., Bartholomew, M.J. (Eds.), Proterozoic Tectonic Evolution of the Grenville Orogen in North America. Geological Society of America Memoir No. 197, pp. 209-241.

Smith, D.R., Noblett, J., Wobus, R.A., Unruh, D., Douglass, J., Beane, R., Davis, C., Goldman, S., Kay, G., Gustvson, B., Saltoun, B., Stewart, J., 1999. Petrology and geochemistry of late-stage intrusions of the A-type, mid-Proterozoic Pikes Peak batholith (Central Colorado, USA): implications for petrogenetic models. Precambrian Res. 98, 271-305.

Southworth, S., 1995. Geologic Map of the Purcellville quadrangle, Loudoun County, Virginia. U.S. Geological Survey Geologic Quadrangle GQ-1755 (Scale 1:24,000).

Spear, F.S., 1993. Metamorphic phase equilibria and pressuretemperature-time paths. Mineral. Soc. Am. Monogr. 1, 799.

Stacey, J.S., Kramers, J.D., 1975. Approximation of terrestrial lead isotope evolution by a two-stage model. Earth Planet. Sci. Lett. 26, 207-226.

Streckeisen, A.J., Le Maitre, R.W., 1979. A chemical approximation to the modal QAPF classification of the igneous rocks. Neues Jahrbuch fuer Mineralogie. Abhandlungen 136, 169-206.

Steiger, R.H., Jäger, E., 1977. Subcommission on geochronology, convention on the use of decay constants in geo- and cosmochronology. Earth Planet. Sci. Lett. 36, 359-362.

Tollo, R.P., Aleinikoff, J.N., 1996. Petrology and U-Pb geochronology of the Robertson River Igneous Suite, Blue Ridge province, Virginia: evidence for multistage magmatism associated with an early episode of Laurentian rifting. Am. J. Sci. 296, 10451090.

Tollo, R.P., Aleinikoff, J.N., Borduas, E.A., Hackley, P.C., Fanning, C.M., 2004a. Petrologic and geochronologic evolution of the Grenville orogen, northern Blue Ridge province, Virginia.Petrologic and geochronologic evolution of the Grenville orogen, northern Blue Ridge province, Virginia. In: Tollo, R.P., Corriveau, L., McLelland, J., Bartholomew, M.J. (Eds.), Proterozoic Tectonic Evolution of the Grenville Orogen in North America. Geological Society of America Memoir No. 197, pp. 647-677.

Tollo, R.P., Aleinikoff, J.N., Bartholomew, M.J., Rankin, D.W., 2004b. Neoproterozoic A-type granitoids of the central and southern Appalachians: intraplate magmatism associated with episodic rifting of the Rodinian supercontinent. Precambrian Res. 128, 3-38.

Tollo, R.P., Bailey, C.M., Borduas, E.A., Aleinikoff, J.N., 2004c. Mesoproterozoic geology of the Blue Ridge province in north-central Virginia: petrologic and structural perspectives on Grenvillian orogenesis and Paleozoic tectonic processes.Mesoproterozoic geology of the Blue Ridge province in north-central Virginia: petrologic and structural perspectives on Grenvillian orogenesis and Paleozoic tectonic processes. In: Southworth, S., Burton, W. (Eds.), Geology of the National Capital Region-Field Trip Guidebook, vol. 1264. U.S. Geological Survey Circular, pp. 17-75.

Turner, S., Arnaud, N., Liu, J., Rogers, N., Hawkesworth, C., Harris, N., Kelley, S., Van Calsteren, P., Deng, W., 1996. Post-collision, shoshonitic volcanism on the Tibetan Plateau: implications for con- 
vective thinning of the lithosphere and the source of ocean island basalts. J. Petrol. 37, 45-71.

Turner, S.P., Foden, J.D., Morrison, R.S., 1992a. Derivation of some A-type magmas by fractionation of basaltic magma: an example from the Padthaway Ridge, South Australia. Lithos 28, 151179.

Turner, S.P., Sandiford, M., Foden, J., 1992b. Some geodynamic and compositional constraints on "postorogenic" magmatism. Geology 20, 931-934.

Vander Auwera, J., Bogaerts, M., Liégeois, J.-P., Demaiffe, D., Wilmart, E., Bolle, O., Duchesne, J.C., 2003. Derivation of the 1.0-0.9 ferro-potassic A-type granitoids of southern Norway by extreme differentiation from basic magmas. Precambrian Res. 124, $107-148$.

Van Schmus, W.R., Bickford, M.E., Anderson, J.L., Bender, E.E., Anderson, R.R., Bauer, P.W., Robertson, J.M., Bowring, S.A., Condie, K.C., Denison, R.E., Gilbert, M.C., Grambling, J.A., Mawer, C.K., Shearer, C.K., Hinze, W.J., Karlstrom, K.E., Kisvarsanyi, E.B., Lidiak, E.G., Reed, J.C., Sims, P.K., Tweto, O., Silver, L., Treves, S.B., Williams, M.L., Wooden, J.L., 1993. Transcontinental Proterozoic provinces. In: Reed Jr., J.C., Bickford, M.E., Houston, R.S., Link, P.K., Rankin, D.W., Sims., P.K., Van Schmus, W.R. (Eds.),Transcontinental Proterozoic provinces. Precambrian: Conterminous U.S. The Geology of North America, vol. C-2. Geological Society of America, Boulder, Colorado, pp. 171-334.

Virginia Division of Mineral Resources, 1993. Geologic Map of Virginia. Virginia Division of Mineral Resources (Scale 1:500,000).

Volkert, R.A., 2004. Mesoproterozoic rocks of the New Jersey Highlands, north-central Appalachians: petrogenesis and tectonic history.Mesoproterozoic rocks of the New Jersey Highlands, northcentral Appalachians: petrogenesis and tectonic history. In: Tollo, R.P., Corriveau, L., McLelland, J., Bartholomew, M.J. (Eds.), Proterozoic Tectonic Evolution of the Grenville Orogen in North America. Geological Society of America Memoir No. 197, pp. 697-728.

Watson, E.B., Harrison, T.M., 1983. Zircon saturation revisited: temperature and compositonal effects in a variety of magma types. Earth Planet. Sci. Lett. 64, 295-304.

Whalen, J.B., Currie, K.L., Chappell, B.W., 1987. A-type granites: geochemical characteristics, discrimination, and petrogenesis. Contrib. Mineral. Petrol. 95, 407-419.

Williams, I.S., 1998. Chapter 1, U-Th-Pb geochronology by ion microprobe.Chapter 1, U-Th-Pb geochronology by ion microprobe. In: McKibben, M.A., Shanks III, W.C., Ridley, W.I. (Eds.), Applications of Microanalytical Techniques to Understanding Mineralizing Processes. Reviews of Economic Geology, vol. 7, pp. 1-35. 